\title{
Regio- and Stereoselective Nickel-Catalyzed Homoallylation of Aldehydes with 1,3-Dienes
}

\author{
Masanari Kimura, Akihiro Ezoe, Masahiko Mori, Keisuke Iwata, \\ Yoshinao Tamaru*
}

\section{Experimental Section}

Distillation were carried out in a Kugelrohr apparatus (SIBATA glass tube oven GTO-350RG). Boiling points are meant to refer to the oven temperature $\left( \pm 1{ }^{\circ} \mathrm{C}\right)$. Microanalyses were performed by the Instrumental Analysis Center of Nagasaki University. Analysis agreed with the calculated values within $\pm 0.4 \%$. High resolution mass spectra (HRMS) were measured with JEOL JMSDX303. Infrared spectra were recorded with a JASCO A-100 or SHIMAZU FTIR-8700 infrared spectrophotometer. ${ }^{1} \mathrm{H}$ and ${ }^{13} \mathrm{C}$ magnetic resonance spectra were measured on JEOL-GX400 instrument with tetramethylsilane as an internal standard. Chemical shift values were given in ppm downfield from the internal standard.

Solvent and Reagents. Tetrahydrofuran and diethyl ether were dried and distilled from benzophenone-sodium immediately prior to use under nitrogen atmosphere. Benzene, dichloromethane, and triethylamine were distilled over calcium hydride. Benzaldehyde was distilled under reduced pressure prior to use. Triethylborane (1.0 $\mathrm{M}$ hexane, KANTO), diethylzinc (1.0 M hexane, KANTO), diisobutylaluminum hydride (DIBAL, KANTO), $\mathrm{Bu}_{4} \mathrm{~N}^{+} \mathrm{F}^{-}$(1 M in THF, Aldrich), 3-methyl-1,3-pentadiene (1i, Z/E = 1.9:1, Tokyo Kasei), 2,6-dimethyl-2,4,6-octatriene (1j, $Z / E=7: 1$, Tokyo Kasei), triisopropylsilyl triflate (TIPS triflate, Aldrich), ethyl (S)-(-)-lactate (Aldrich), Ni(acac) 2 (Aldrich), and $\mathrm{Ni}(\mathrm{cod})_{2}(\mathrm{KANTO})$ were used without further purification.

\section{Preparation of starting materials:}


Aldehydes: 2-(Triisopropylsiloxy)acrolein: ${ }^{1}$ Dimeric 1,3-(dihydroxy)-2-propanone (2.7 g, $15 \mathrm{mmol}$ ) and camphor-10-sulfonic acid (34.8 $\mathrm{mg}, 0.15 \mathrm{mmol})$ were dissolved in dioxane $(100 \mathrm{~mL})$ at $60^{\circ} \mathrm{C}$ under argon. To this solution was added trimethyl orthoacetate $(19 \mathrm{~mL}$, $150 \mathrm{mmol}$ ) at the same temperature. The resulting solution was stirred at the same temperature for $20 \mathrm{~h}$ and then concentrated in vacuo. The residue was distilled $\left(100^{\circ} \mathrm{C} / 10\right.$ $\mathrm{mmHg}$ ) to give 2-methyl-2-methoxy-1,3-dioxan-5-one (50\%). 2-Methyl-2-methoxy1,3-dioxan-5-one (731 mg, $5 \mathrm{mmol})$ and triethylamine $(1.2 \mathrm{~mL}, 8.5 \mathrm{mmol})$ were dissolved in benzene $(10 \mathrm{~mL})$. To this solution was added TIPS triflate $(16.1 \mathrm{~mL}, 60 \mathrm{mmol})$ dropwise at room temperature. The resulting solution was stirred at room temperature for $18 \mathrm{~h}$. The reaction mixture was quenched with water and extracted with ether $(3 \times 30 \mathrm{~mL})$. The combined extracts were washed with brine $(50 \mathrm{~mL})$. The organic phase was dried $\left(\mathrm{MgSO}_{4}\right)$ and the solvent was removed in vacuo. The residue was purified by column chromatography over silica gel (hexane/ethyl acetate $=64 / 1, \mathrm{v} / \mathrm{v}$ ) to provide the titled aldehyde $(62 \%)$.

(2S)-2-(t-Butyldimethylsiloxy)propionaldehyde: Freshly distilled ethyl $(S)$-(-)-lactate (3 $\mathrm{mL}, 26 \mathrm{mmol})$, imidazole $(2.66 \mathrm{~g}, 39 \mathrm{mmol})$, and $t \mathrm{BuMe}_{2} \mathrm{SiCl}(4.85 \mathrm{~g}, 32 \mathrm{mmol})$ were dissolved in $N, N$-dimethylformamide $(26 \mathrm{~mL})$. The resulting solution was stirred at room temperature for $1 \mathrm{~h}$. The solution was diluted with hexane $(150 \mathrm{~mL})$ and washed with water. The organic phase was dried $\left(\mathrm{MgSO}_{4}\right)$ and concentrated in vacuo. The residue was distilled $\left(90{ }^{\circ} \mathrm{C} / 20 \mathrm{mmHg}\right.$ ) to give ethyl 2-( $t$-butyldimethylsiloxy)propanoate (90\%). A solution of 2-(t-butyldimethylsiloxy)propanoate $(3.48 \mathrm{~g}, 15 \mathrm{mmol})$ in ether $(20 \mathrm{~mL})$ was cooled to $-78^{\circ} \mathrm{C}$. To this solution was added DIBAL (22.5 mmol, $1.0 \mathrm{M}$ hexane solution) dropwise at the same temperature. The resulting solution was stirred at same temperature for $10 \mathrm{~min}$. Into this solution were added methanol $(0.8 \mathrm{~mL})$ and water $(2.3 \mathrm{~mL})$. The mixture was stirred at room temperature for $1 \mathrm{~h}$. The mixture were passed through a short 
pad of celite, and the solvent was removed in vacuo. The residue was purified by column chromatography over silica gel (hexane/ethyl acetate $=16 / 1, \mathrm{v} / \mathrm{v}$ ) to provide the titled aldehyde $(62 \%)$.

(2S)-2-Benzyloxypropionaldehyde: Into a round-bottom flask was placed $\mathrm{NaH}$ (3 g, $60 \%$ dispersion in oil, $75 \mathrm{mmol})$, which was triturated with hexane $(2 \times 5 \mathrm{~mL})$. To the flask was added $N, N$-dimethylformamide $(40 \mathrm{~mL})$ and the mixture was cooled to $0{ }^{\circ} \mathrm{C}$. To this solution was added dropwise a solution of ethyl $(S)-(-)$-lactate $(3.4 \mathrm{~mL}, 30 \mathrm{mmol})$ in dimethylformamide $(40 \mathrm{~mL})$. After $30 \mathrm{~min}$ benzyl bromide $(9.0 \mathrm{~mL}, 75 \mathrm{mmol})$ was added dropwise and the resulting solution was stirred at same temperature for $3 \mathrm{~h}$. Into this solution were added ether $(100 \mathrm{~mL})$ and sat. $\mathrm{NH}_{4} \mathrm{Cl}(30 \mathrm{~mL})$, and the organic layer was separated and washed with brine. The organic phase was dried over $\mathrm{MgSO}_{4}$ and the solvent was removed in vacuo. The residue was purified by column chromatography over silica gel (hexane/ethyl acetate $=16 / 1, \mathrm{v} / \mathrm{v}$ ) to provide ethyl $(2 \mathrm{~S})$-2-benzyloxypropionate $(85 \%)$, which was reduced with DIBAL as described above to give the titled aldehyde in $45 \%$ yield (column chromatography over silica gel; hexane/ethyl acetate $=16 / 1, \mathrm{v} / \mathrm{v}$ ).

Dienes: $(\boldsymbol{E})$-1-Phenyl-1,3-butadiene (1g) : To a mixture of $\mathrm{Mg}(10.9 \mathrm{mg}, 450 \mathrm{mmol})$ and benzaldehyde $(15.3 \mathrm{~mL}, 150 \mathrm{mmol})$ in ether $(20 \mathrm{~mL})$, kept at $0{ }^{\circ} \mathrm{C}$, allyl bromide $(19.4 \mathrm{~mL}$, $225 \mathrm{mmol})$ in ether $(150 \mathrm{~mL})$ was added dropwise over $1 \mathrm{~h}$. The reaction mixture was allowed to warm to room temperature, and stirred for $1 \mathrm{~h}$ at the same temperature. The reaction mixture was filtered, and the filtrate was washed with $2 \mathrm{M} \mathrm{HCl}$ and with brine, then dried $\left(\mathrm{MgSO}_{4}\right)$, concentrated in vacuo to give an oil, which was purified by distillation $\left(100{ }^{\circ} \mathrm{C} / 1 \mathrm{mmHg}\right)$ to give 1-phenyl-3-buten-1-ol (90\%). A mixture of 1-phenyl3-buten-1-ol (6.22g, $42 \mathrm{mmol}), \mathrm{NaHSO}_{4}(0.29 \mathrm{~g}, 5 \mathrm{~mol} \%)$, and hydroquinone (46 mg, 1 mol\%) was heated at $120{ }^{\circ} \mathrm{C}$ under reduced pressure $(30 \mathrm{mmHg})$ with kugelrohr. Once dehydration started, the pressure was reduced $(10 \mathrm{mmHg})$ to distill out $\mathbf{1 g}$ continuously 
$(43 \%)$.

2-(Triisopropylsilyloxy)-1,3-butadiene (1r): Freshly distilled methyl vinyl ketone (4.2 $\mathrm{mL}, 50 \mathrm{mmol})$ and triethylamine $(10.4 \mathrm{~mL}, 75 \mathrm{mmol})$ were dissolved in benzene $(50 \mathrm{~mL})$. To this solution was added TIPS triflate $(16.1 \mathrm{~mL}, 60 \mathrm{mmol})$ dropwise at $0{ }^{\circ} \mathrm{C}$. The mixture was stirred at room temperature for $2 \mathrm{~h}$ and then diluted with hexane $(50 \mathrm{~mL})$. The mixture was washed with cold sat. $\mathrm{NaHCO}_{3}(2 \times 50 \mathrm{~mL})$ and brine $(50 \mathrm{~mL})$, dried $\left(\mathrm{MgSO}_{4}\right)$, and concentrated in vacuo to give an oil, which was distilled $\left(135{ }^{\circ} \mathrm{C} / 15 \mathrm{mmHg}\right)$ to give 1r (18\%).

(E)-1-Phenyl-3-(triisopropylsilyloxy)-1,3-butadiene (1t) was prepared similarly from trans-4-phenyl-3-buten-2-one in $62 \%$ yield $\left(145^{\circ} \mathrm{C} / 0.2 \mathrm{mmHg}\right)$.

(E)-1-(Triisopropylsilyloxy)-1,3-butadiene (1u): Freshly distilled crotonaldehyde (5.8 mL, $71.8 \mathrm{mmol})$ and triethylamine $(13.7 \mathrm{~mL}, 98.3 \mathrm{mmol})$ were dissolved in dichloromethane $(30 \mathrm{~mL})$. To this solution was added TIPS triflate $(11 \mathrm{~mL}, 40.9 \mathrm{mmol})$ dropwise at $0{ }^{\circ} \mathrm{C}$, then the mixture was heated at reflux for $5 \mathrm{~h}$. The mixture was diluted with ether (120 $\mathrm{mL})$ and washed with cold sat. $\mathrm{NaHCO}_{3}(2 \times 50 \mathrm{~mL})$ and brine $(50 \mathrm{~mL})$. The organic phase was dried $\left(\mathrm{MgSO}_{4}\right)$ and the solvent was removed in vacuo. The residue was distilled $\left(110^{\circ} \mathrm{C} / 8 \mathrm{mmHg}\right)$ to give the $1 \mathbf{u}(91 \%)$.

(E)-1-(Triisopropylsilyloxy)-3-methyl-1,3-butadiene (1v) was prepared in a similar way from 3-methyl-2-butenal in $92 \%$ yield $\left(100{ }^{\circ} \mathrm{C} / 1 \mathrm{mmHg}\right)$.

1,3-Bis(trimethylsiloxy)-1-methoxybuta-1,3-diene (1x): Freshly distilled methyl acetoacetate $(5.4 \mathrm{~mL}, 50 \mathrm{mmol})$ and triethylamine $(8.4 \mathrm{~mL}, 60 \mathrm{~mL})$ were dissolved in hexane $(100 \mathrm{~mL})$. To this solution was added trimethylsilyl chloride $(7.0 \mathrm{~mL}, 55 \mathrm{mmol})$ dropwise. The resulting mixture was stirred at room temperature for $18 \mathrm{~h}$. The mixture were passed through a short pad of celite and the filtrate was concentrated in vacuo. The residue was distilled $\left(100{ }^{\circ} \mathrm{C} / 20 \mathrm{mmHg}\right)$ to give methyl 3-(trimethylsiloxy)-2-butenoate 
(87\%). Methyl 3-(trimethylsiloxy)-2-butenoate $(3.77 \mathrm{~g}, 20 \mathrm{mmol})$ was added dropwise to a freshly prepared solution of LDA $(22 \mathrm{mmol})$ in $\mathrm{THF}$ at $-78{ }^{\circ} \mathrm{C}$. After $30 \mathrm{~min}$, trimethylsilyl chloride $(3.4 \mathrm{~mL}, 27 \mathrm{mmol})$ was slowly added and the reaction mixture was warmed to $0{ }^{\circ} \mathrm{C}$. After $1 \mathrm{~h}$, all volatile materials were removed in vacuo at room temperature, and the residue was washed with hexane. The hexane extracts were concentrated in vacuo and the residue was distilled $\left(50{ }^{\circ} \mathrm{C} / 0.01 \mathrm{mmHg}\right)$ to give $\mathbf{1 x}(97 \%)$.

\section{Typical procedure for the homoallylation of aldehydes with dienes (run 2, Table 1):}

Into a $\mathrm{N}_{2}$-purged flask containing $\mathrm{Ni}(\mathrm{acac})_{2}(25.7 \mathrm{mg}, 0.1 \mathrm{mmol})$ were introduced successively THF (5 mL), isoprene (1b, $0.4 \mathrm{~mL}, 4 \mathrm{mmol})$, benzaldehyde (106 mg, $1 \mathrm{mmol})$, and $\mathrm{Et}_{3} \mathrm{~B}$ (2.4 mL, $1 \mathrm{M}$ in hexane) via syringe. The homogeneous mixture was stirred at room temperature for $35 \mathrm{~h}$, during which the reaction was monitored by TLC. After dilution with ethyl acetate $(30 \mathrm{~mL})$, the mixture was washed successively with $2 \mathrm{~N}-\mathrm{HCl}$, sat. $\mathrm{NaHCO}_{3}$, and brine, and then dried $\left(\mathrm{MgSO}_{4}\right)$ and concentrated in vacuo. The residual oil was subjected to column chromatography over silica gel (hexane/ethyl acetate $=16 / 1$, v/v) to give an analytically pure sample of $2.2(159 \mathrm{mg}, 90 \%)$.

1-Phenyl-4-pentenol (2.1): IR (neat) 3360 (s), 2945 (s), 1645 (m), 1459 (s), 1060 (s), 1020 (s), 916 (s), 760 (m), 698 (s) cm-1; ${ }^{1} \mathrm{H}$ NMR (400 MHz, CDCl 3$), \delta 1.80$ (m, $1 \mathrm{H}$, coalescing to $\mathrm{dd}, J=5.1,13.6 \mathrm{~Hz}$ by irradiation at 2.14$), 1.86(\mathrm{~d}, J=2.9 \mathrm{~Hz}, 1 \mathrm{H}), 1.90(\mathrm{~m}, 1 \mathrm{H}$, coalescing to br dd, $J=7.3,13.6 \mathrm{~Hz}$ by irradiation at 2.14$), 2.12(\mathrm{~m}, 1 \mathrm{H}$, coalescing to ddd, $J=6.6,8.2,14.3 \mathrm{~Hz}$ by irradiation at 5.84$), 2.17(\mathrm{~m}, 1 \mathrm{H}$, coalescing to ddd, $J=6.6,8.2$, 14.3 Hz by irradiation at 5.84), $4.70(\mathrm{ddd}, J=2.9,5.1,7.3 \mathrm{~Hz}, 1 \mathrm{H}), 4.98(\mathrm{br} \mathrm{d}, J=10.3 \mathrm{~Hz}$, $1 \mathrm{H}), 5.04$ (br d, $J=16.9 \mathrm{~Hz}, 1 \mathrm{H}), 5.84$ (ddt, $J=10.3,16.9,6.6 \mathrm{~Hz}, 1 \mathrm{H}), 7.22-7.40$ (m, 5 $\mathrm{H}$ ); HRMS, calcd for $\mathrm{C}_{11} \mathrm{H}_{14} \mathrm{O}: 162.1045$, found $\mathrm{m} / z$ (relative intensity) $162.1050\left(\mathrm{M}^{+}, 8\right)$, 144 (8), 107 (100), 77 (17). Anal. calcd for $\mathrm{C}_{11} \mathrm{H}_{14} \mathrm{O}$ : C, 81.44; H, 8.70, found: C, 81.31; 
H, 8.84.

3-Methyl-1-phenyl-4-pentenol (2.2): a mixture of 1,3-anti and syn = $15: 1$; IR (neat) 3350 (s), 2935 (m), 1642 (w), 1450 (m), 1028 (s), 908 (s), 692 (s) cm-1, ${ }^{1} \mathrm{H}$ NMR (400 MHz, $\left.\mathrm{CDCl}_{3}\right), \delta 1.03(\mathrm{~d}, J=6.4 \mathrm{~Hz}, 3 \mathrm{H}), 1.64(\mathrm{dt}, J=13.8,6.4 \mathrm{~Hz}, 1 \mathrm{H}), 1.85(\mathrm{dt}, J=13.8,7.8$ $\mathrm{Hz}, 1 \mathrm{H}), 1.98$ (br s, $1 \mathrm{H}), 2.22$ (tquint, $J=7.8,6.4 \mathrm{~Hz}, 1 \mathrm{H}), 4.72(\mathrm{dd}, J=6.4,7.8 \mathrm{~Hz}, 1 \mathrm{H}$ ), 4.97 (br d, $J=10.3 \mathrm{~Hz}, 1 \mathrm{H}$ ), 5.01 (br d, $J=17.3 \mathrm{~Hz}, 1 \mathrm{H}$ ), 5.78 (ddd, $J=7.8,10.3,17.3 \mathrm{~Hz}$, $1 \mathrm{H}), 7.25$ - $7.35(\mathrm{~m}, 5 \mathrm{H}) ;{ }^{13} \mathrm{C} \mathrm{NMR}\left(100 \mathrm{MHz}, \mathrm{CDCl}_{3}\right)$, 1,3-anti isomer: $\delta 20.4$, 35.1, $46.0,73.1,113.2,126.0,127.6,128.1,144.6,144.7 ; 1,3-s y n$ isomer: $\delta 22.5,34.0,36.9,74.3$, 110.2, 125.9, 126.0, 127.6, 144.7, 145.4; HRMS calcd for $\mathrm{C}_{12} \mathrm{H}_{16} \mathrm{O}: 176.1201$, found $\mathrm{m} / z$ (relative intensity) $176.1220\left(\mathrm{M}^{+}, 5\right), 158(2), 107$ (100), 91 (3), 77 (14). Anal. calcd for $\mathrm{C}_{12} \mathrm{H}_{16} \mathrm{O}: \mathrm{C}, 81.77 ; \mathrm{H}, 9.15$, found $\mathrm{C}, 81.61 ; \mathrm{H}, 9.23$.

anti-1-Phenyl-3-(2-trimethylsilylmethyl)-4-pentenol (2.3): IR (neat) 3350 (s), 2950 (s), 1450 (m), 1250 (s), 1040 (m), 990 (m), 900 (s), 850 (s), 830 (s), 750 (s), 690 (s) cm-1; ${ }^{1} \mathrm{H}$ NMR (400 MHz, $\left.\mathrm{CDCl}_{3}\right), \delta 0.04(\mathrm{~s}, 9 \mathrm{H}), 0.68(\mathrm{dd}, J=8.4,14.7 \mathrm{~Hz}, 1 \mathrm{H}), 0.80(\mathrm{dd}, J=5.9$, $14.7 \mathrm{~Hz}, 1 \mathrm{H}), 1.85$ (ddd, $J=5.1,6.7,13.7 \mathrm{~Hz}, 1 \mathrm{H}), 1.93(\mathrm{ddd}, J=6.7,9.2,13.7 \mathrm{~Hz}, 1 \mathrm{H})$, $2.10(\mathrm{~d}, J=2.2 \mathrm{~Hz}, 1 \mathrm{H}), 2.24(\mathrm{~m}, 1 \mathrm{H}), 4.79(\mathrm{dt}, J=2.2,6.7 \mathrm{~Hz}, 1 \mathrm{H}), 5.07(\mathrm{dd}, J=1.8$, $10.3 \mathrm{~Hz}, 1 \mathrm{H}), 5.08(\mathrm{dd}, J=1.8,17.3 \mathrm{~Hz}, 1 \mathrm{H}), 5.80(\mathrm{ddd}, J=9.2,10.3,17.3 \mathrm{~Hz}, 1 \mathrm{H})$. Anal. calcd for $\mathrm{C}_{15} \mathrm{H}_{24} \mathrm{OSi}$ : C, 72.52, H, 9.74, found C, 72.36, H, 9.83.

anti-1,3-Diphenyl-4-pentenol (2.4): IR (neat) 3369 (s), 2916 (m), 1492 (m), 1454 (s), 997 (m), 914 (s), 752 (s), 700 (s) cm-1; ${ }^{1} \mathrm{H}$ NMR (400 MHz, CDCl 3 ), $\delta 1.85$ (d, J=4.0 Hz, $\left.1 \mathrm{H}\right)$, $2.06(\mathrm{ddd}, J=4.0,9.2,13.9 \mathrm{~Hz}, 1 \mathrm{H}), 2.23(\mathrm{ddd}, J=6.3,8.8,13.9 \mathrm{~Hz}, 1 \mathrm{H}), 3.55(\mathrm{~m}, 1 \mathrm{H})$, 4.50 (dt, $J=8.8,4.0 \mathrm{~Hz}, 1 \mathrm{H}), 5.04$ (br d, $J=9.9 \mathrm{~Hz}, 1 \mathrm{H}), 5.05$ (br d, $J=17.6 \mathrm{~Hz}, 1 \mathrm{H}$ ), 6.02 (ddd, $J=7.3,9.9,17.6 \mathrm{~Hz}, 1 \mathrm{H}), 7.20-7.35$ (m, $10 \mathrm{H}$ ); HRMS calcd for $\mathrm{C}_{18} \mathrm{H}_{18} \mathrm{O}$ : 238.1358, found $m / z$ (relative intensity) $239\left(\mathrm{M}^{+}+1,7\right), 238.1379\left(\mathrm{M}^{+}, 38\right), 221(18), 220$ (100), 205 (27). 
anti-7-Methyl-1-phenyl-3-vinyl-6-octenol (2.5): IR (neat) 3335 (s), 2950 (s), 1640 (w), 1450 (s), 1370 (m), 1000 (m), 905 (s), 750 (m), 690 (s) cm-1; ${ }^{1} \mathrm{H}$ NMR (400 MHz, C 6 D $\left.\mathrm{D}_{6}\right) \delta$ $1.22(\mathrm{dm}, J=14.8 \mathrm{~Hz}, 1 \mathrm{H}), 1.41(\mathrm{dm}, J=14.8 \mathrm{~Hz}, 1 \mathrm{H}), 1.50(\mathrm{~s}, 3 \mathrm{H}), 1.64(\mathrm{~s}, 3 \mathrm{H}), 1.70$ (ddd, $J=6.9,8.2,13.7 \mathrm{~Hz}, 1 \mathrm{H}), 1.80(\mathrm{ddd}, J=6.9,8.9,13.7 \mathrm{~Hz}, 1 \mathrm{H}), 1.88(\mathrm{~m}, 1 \mathrm{H}$, coalescing to br dd, $J=7.5,14.7 \mathrm{~Hz}$ by irradiation at 1.41), $2.00(\mathrm{~m}, 1 \mathrm{H}$, coalescing to br $\mathrm{dd}, J=7.5,14.7 \mathrm{~Hz}$, by irradiation at 1.41$), 2.04(\mathrm{dtm}, J=8.2,8.9 \mathrm{~Hz}, 1 \mathrm{H}), 4.55(\mathrm{t}, J=6.9$ $\mathrm{Hz}, 1 \mathrm{H}), 4.94(\mathrm{dd}, J=2.2,16.9 \mathrm{HZ}, 1 \mathrm{H}), 4.97$ (dd, $J=2.2,10.3 \mathrm{~Hz}, 1 \mathrm{H}), 5.11$ (br t, $J=$ $7.5 \mathrm{~Hz}, 1 \mathrm{H}), 5.54(\mathrm{ddd}, J=8.9,10.3,16.9 \mathrm{~Hz}, 1 \mathrm{H}), 7.02-7.35(\mathrm{~m}, 5 \mathrm{H}) ;{ }^{13} \mathrm{C}$ NMR (100 $\left.\mathrm{MHz}, \mathrm{CDCl}_{3}\right) \delta 17.7,25.4,25.6,35.3,41.3,44.4,73.4,115.2,124.4,126.1,127.6,128.5$, 131.5, 143.2, 144.5; HRMS calcd for $\mathrm{C}_{17} \mathrm{H}_{24} \mathrm{O}$ : 244.1827, found $\mathrm{m} / \mathrm{z}$ (relative intensity) $244.1851\left(\mathrm{M}^{+}, 19\right), 226$ (41), 201 (45), 159 (65), 107 (100), 77 (25). Anal. calcd for $\mathrm{C}_{17} \mathrm{H}_{24} \mathrm{O}: \mathrm{C}, 83.55 ; \mathrm{H}, 9.90$, found $\mathrm{C}, 83.58 ; \mathrm{H}, 9.97$.

(4E)-1-Phenyl-4-hexenol (2.6) : IR (neat) 3350 (s), 2940 (s), 1700 (w), 1450 (s), 1310 (m), 1200 (m), 1050 (m), 960 (s), 750 (s), 690 (s) cm-1; ${ }^{1} \mathrm{H}$ NMR (400 MHz, $\mathrm{C}_{6} \mathrm{D}_{6}$ ) $\delta 1.39$ (br s, $1 \mathrm{H}), 1.56$ (br d, $J=4.8 \mathrm{~Hz}, 3 \mathrm{H}$ ), 1.66 (dddd, $J=5.5,6.6,9.2,14.6 \mathrm{~Hz}, 1 \mathrm{H}$ ), 1.78 (dddd, $J$ $=5.9,7.7,8.6,14.6 \mathrm{~Hz}), 2.01(\mathrm{~m}, 1 \mathrm{H}$, coalescing to br ddd, $J=6.6,8.6,15.0 \mathrm{~Hz}$ by irradiation at 5.34), $2.09(\mathrm{~m}, 1 \mathrm{H}$, coalescing to br ddd, $J=5.9,9.2,15.0 \mathrm{~Hz}$ by irradiation at 5.34), $4.41(\mathrm{dd}, J=5.5,7.7 \mathrm{~Hz}, 1 \mathrm{H}), 5.34(\mathrm{~m}, 1 \mathrm{H}$, coalescing to ddd, $J=5.1,6.2,15.2$ $\mathrm{Hz}$ by irradiation at 1.56), $5.39(\mathrm{~m}, 1 \mathrm{H}$, coalescing to $\mathrm{d}, J=15.2 \mathrm{~Hz}$, by irradiation at 1.56$)$, 7.22-7.40 (m, $5 \mathrm{H}) ;{ }^{13} \mathrm{C} \mathrm{NMR}\left(100 \mathrm{MHz}, \mathrm{CDCl}_{3}\right) \delta 17.9,28.9,38.8,74.1,125.6,125.9$, 127.5, 128.4, 130.6, 144.7; HRMS calcd for $\mathrm{C}_{12} \mathrm{H}_{16} \mathrm{O}$ : 176.1201, found $\mathrm{m} / \mathrm{z}$ (relative intensity) $176.1230\left(\mathrm{M}^{+}, 23\right), 158$ (18), 120 (19), 107 (100), 79 (42). Anal. calcd for $\mathrm{C}_{12} \mathrm{H}_{16} \mathrm{O}: \mathrm{C}, 81.77 ; \mathrm{H}, 9.15$, found $\mathrm{C}, 81.42 ; \mathrm{H}, 9.20$.

(4E)-1,5-Diphenyl-4-pentenol (2.7): IR (neat) 3350 (s), 3060 (m), 3030 (m), 2940 (m), $1950(\mathrm{w}), 1880(\mathrm{w}), 1810(\mathrm{w}), 1600(\mathrm{w}), 1500$ (m), 1450 (m), 1060 (m), 1030 (m), 960 (s), 
$740(\mathrm{~s}), 695(\mathrm{~s}) \mathrm{cm}^{-1} ;{ }^{1} \mathrm{H} \mathrm{NMR}\left(400 \mathrm{MHz}, \mathrm{CDCl}_{3}\right) \delta 1.87(\mathrm{~m}, 1 \mathrm{H}$, coalescing to ddd, $J=$ 6.4, 8.6, 13.2 $\mathrm{Hz}$ by irradiation at 4.72), $1.91(\mathrm{~d}, J=2.9 \mathrm{~Hz}, 1 \mathrm{H}), 1.96(\mathrm{~m}, 1 \mathrm{H}$, coalescing to ddd, $J=6.4,8.6,13.2 \mathrm{~Hz}$ by irradiation at 4.72$), 2.22-2.34(\mathrm{~m}, 2 \mathrm{H}$, coalescing to ddm, $J$ $=6.4,8.6 \mathrm{~Hz}$ by irradiation at 6.21$), 4.72(\mathrm{dd}, J=5.3,7.7 \mathrm{~Hz}, 1 \mathrm{H}), 6.21(\mathrm{dt}, J=15.8,7.0$ $\mathrm{Hz}, 1 \mathrm{H}), 6.39(\mathrm{~d}, J=15.8 \mathrm{~Hz}, 1 \mathrm{H}), 7.12-7.38(\mathrm{~m}, 5 \mathrm{H})$; HRMS calcd for $\mathrm{C}_{17} \mathrm{H}_{18} \mathrm{O}$ : 238.1358, found $\mathrm{m} / \mathrm{z}$ (relative intensity) 238.1360 $\left(\mathrm{M}^{+}, 68\right), 220$ (34), 133 (92), 120 (100), 107 (97), 77 (43).

(4E)-1,3-anti-3-Methyl-1-phenyl-4-hexenen-1-ol (2.8): IR (neat) 3365 (s), 2965 (s), 2940 (s), 1450 (m), 1370 (w), 1030 (w), 960 (s), 690 (s) cm-1; ${ }^{1} \mathrm{H}$ NMR (400 MHz, CDCl $\left.{ }_{3}\right) \delta$ $0.99(\mathrm{~d}, J=6.6 \mathrm{~Hz}, 3 \mathrm{H}), 1.64(\mathrm{dt}, J=13.7,6.0 \mathrm{~Hz}, 1 \mathrm{H}), 1.67(\mathrm{~d}, J=5.5 \mathrm{~Hz}, 3 \mathrm{H}), 1.81$ (dt, $J=13.7,7.9 \mathrm{~Hz}, 1 \mathrm{H}), 2.04(\mathrm{~d}, J=2.6 \mathrm{~Hz}, 1 \mathrm{H}), 2.16(\mathrm{~m}, 1 \mathrm{H}$, coalescing to ddd $J=6.0,7.2$, $7.9 \mathrm{~Hz}$ by irradiation at 0.99$), 4.73(\mathrm{ddd}, J=2.6,6.0,7.9 \mathrm{~Hz}, 1 \mathrm{H}), 5.38(\mathrm{dd}, J=7.2,15.4$ $\mathrm{Hz}, 1 \mathrm{H}), 5.44(\mathrm{dq}, J=15.4,5.5 \mathrm{~Hz}, 1 \mathrm{H}), 7.24-7.38(\mathrm{~m}, 5 \mathrm{H}) ;{ }^{13} \mathrm{C} \mathrm{NMR}(100 \mathrm{MHz}$, $\left.\mathrm{CDCl}_{3}\right) \delta 17.9,21.2,34.3,46.5,73.3,123.9,126.1,127.5,128.4,137.4,144.8 ; \mathrm{MS} \mathrm{m} / \mathrm{z}$ (relative intensity) $190\left(\mathrm{M}^{+}, 28\right), 172(42), 107(90), 91$ (23), 77 (100). Anal. calcd for $\mathrm{C}_{13} \mathrm{H}_{18} \mathrm{O}: \mathrm{C}, 82.06$; H, 9.53, found C, 82.13; H, 9.76.

2,3-Dimethyl-1-Phenyl-4-Pentenol (2.9): a mixture of 1,2-syn-2,3-anti- and 1,2-anti-2,3syn-2.9 = 8:1; IR (neat) 3425 (s), 2975 (s), 1650 (w), 1500 (m), 1460 (s), 1380 (m), 1120 (w), 1010 (s), 910 (s), 760 (s), 700 (s) cm-1; ${ }^{1} \mathrm{H}$ NMR (400 MHz, CDCl 3 ) 1,2-syn-2,3-anti isomer: $\delta 0.89(\mathrm{~d} J=7.0 \mathrm{~Hz}, 3 \mathrm{H}), 1.04(\mathrm{~d}, J=6.6 \mathrm{~Hz}, 3 \mathrm{H}), 1.73(\mathrm{~m}, 1 \mathrm{H}$, coalescing to t, $J$ $=5.9 \mathrm{~Hz}$ by irradiation at 0.89$), 2.16(\mathrm{~m}, 1 \mathrm{H}$, coalescing to $\mathrm{dd}, J=5.9,8.4 \mathrm{~Hz}$ by irradiation at 1.04), $4.66(\mathrm{~d}, J=5.9 \mathrm{~Hz}, 1 \mathrm{H}), 4.96(\mathrm{dd}, J=1.3,17.2 \mathrm{~Hz}, 1 \mathrm{H}), 5.00(\mathrm{dd}, J=$ 1.3, 10.3 Hz, $1 \mathrm{H}), 5.78$ (ddd, $J=8.4,10.3,17.2 \mathrm{~Hz}, 1 \mathrm{H}), 7.23-7.36(\mathrm{~m}, 5 \mathrm{H}) ;{ }^{13} \mathrm{C}$ NMR (100 MHz, CDCl3), 1,2-syn-2,3-anti isomer : $\delta$ 9.5, 19.1, 40.4, 45.4, 76.7, 114.7, 126.3, 127.2, 128.2, 141.8, 144.1; ${ }^{1} \mathrm{H} \mathrm{NMR}\left(400 \mathrm{MHz} \mathrm{CDCl}_{3}\right)$ 1,2-anti-2,3-syn isomer: $\delta 0.53$ (d, 
$J=7.0 \mathrm{~Hz}, 3 \mathrm{H}), 1.02(\mathrm{~d}, J=6.6 \mathrm{~Hz}, 3 \mathrm{H}), 1.61(\mathrm{br} \mathrm{s}, 1 \mathrm{H}), 1.92(\mathrm{ddq}, J=4.2,9.0,7.0 \mathrm{~Hz}, 1$ H), $2.74(\mathrm{dtq}, J=4.2,1.3,6.6 \mathrm{~Hz}, 1 \mathrm{H}), 4.46(\mathrm{br} \mathrm{d}, J=9.0 \mathrm{~Hz}, 1 \mathrm{H}), 5.04(\mathrm{dt}, J=10.3,1.3$ $\mathrm{Hz}, 1 \mathrm{H}), 5.06$ (dt, $J=17.2,1.3 \mathrm{~Hz}, 1 \mathrm{H}), 5.89$ (ddd, $J=6.6,10.3,17.2 \mathrm{~Hz}, 1 \mathrm{H}), 7.23-7.36$ $(\mathrm{m}, 5 \mathrm{H}) ;{ }^{13} \mathrm{C} \mathrm{NMR}\left(100 \mathrm{MHz}, \mathrm{CDCl}_{3}\right)$ 1,2-anti-2,3-syn isomer $\delta 10.9,13.1,37.1,44.3$, 76.6, 113.3, 126.9, 127.7, 128.4, 141.8, 144.2; HRMS calcd for $\mathrm{C}_{13} \mathrm{H}_{18} \mathrm{O}$ : 190.1358, found $m / z$ (relative intensity) $190.1366\left(\mathrm{M}^{+}, 6\right), 134$ (15), 107 (100), 84 (14), 77 (10). Anal calcd for $\mathrm{C}_{13} \mathrm{H}_{18} \mathrm{O}$ : C, 82.06; H, 9.53, found: C, 81.74, H, 9.65.

1,2-syn-2,3-anti-2,3,7-Trimethyl-1-phenyl-4,6-octadienol (2.10): a mixture of 1,2-syn-2,3-anti- and 1,2-anti-2,3-syn-2.10 = 15:1; IR (neat) 3420 (s), 3300 (s), 1460 (m), $1380(\mathrm{~m}), 1100$ (s), 700 (s) cm-1; ${ }^{1} \mathrm{H}$ NMR (400 MHz, $\left.\mathrm{CDCl}_{3}\right) \delta 0.91(\mathrm{~d}, J=7.0 \mathrm{~Hz}, 3 \mathrm{H})$, 1.06 (d, $J=7.0 \mathrm{~Hz}, 3 \mathrm{H}), 1.75$ (br s, $3 \mathrm{H}), 1.76$ (ddq, $J=4.9,6.0,7.0 \mathrm{~Hz}, 1 \mathrm{H}), 1.77$ (br s, 3 H), $1.84(\mathrm{~d}, J=3.2 \mathrm{~Hz}, 1 \mathrm{H}), 2.22(\mathrm{ddq}, J=4.9,8.9,7.0 \mathrm{~Hz}, 1 \mathrm{H}), 4.68(\mathrm{dd}, J=3.2,6.0 \mathrm{~Hz}$, $1 \mathrm{H}), 5.51(\mathrm{dd}, J=8.9,15.0 \mathrm{~Hz}, 1 \mathrm{H}), 5.81(\mathrm{br} \mathrm{d}, J=11.0 \mathrm{~Hz}, 1 \mathrm{H}), 6.61(\mathrm{dd}, J=11.0,15.0$ $\mathrm{Hz}, 1 \mathrm{H}), 7.22-7.40(\mathrm{~m}, 5 \mathrm{H})$; HRMS, calcd for $\mathrm{C}_{17} \mathrm{H}_{24} \mathrm{O}: 244.1827$, found $\mathrm{m} / \mathrm{z}$ (relative intensity) $244.1807\left(\mathrm{M}^{+}, 48\right), 226$ (26), 211 (24), 137 (25), 134 (37), 105 (100). Anal. calcd for $\mathrm{C}_{17} \mathrm{H}_{24} \mathrm{O}$ : C, 83.55; H, 9.90, found: C, 83.45; H, 9.95 .

1,3-anti-3,4-Dimethyl-1-phenyl-4-pentenol (2.11): IR (neat) 3370 (s), 1650 (m), 1455 (s), 1376 (m), 1062 (m), 1030 (s), 880 (s), 760 (m), 698 (s) cm ${ }^{-1} ;{ }^{1} \mathrm{H}$ NMR (400 MHz, CDCl${ }_{3}$ ) $\delta 1.04(\mathrm{~d}, J=7.0 \mathrm{~Hz}, 3 \mathrm{H}), 1.63(\mathrm{dt}, J=13.9,7.0 \mathrm{~Hz}, 1 \mathrm{H}), 1.73($ br s, $3 \mathrm{H}), 1.97$ (dt, $J=$ 13.9, 7.0 Hz, 1 H), 2.02 (br s, $1 \mathrm{H}), 2.26$ (sex, $J=7.0 \mathrm{~Hz}, 1 \mathrm{H}), 4.68$ (br t, $J=7.0 \mathrm{~Hz}, 1 \mathrm{H}$ ), 4.75 (br s, $2 \mathrm{H}), 7.24-7.38$ (m, $5 \mathrm{H}) ;{ }^{13} \mathrm{C} \mathrm{NMR}\left(100 \mathrm{MHz}, \mathrm{CDCl}_{3}\right) \delta 19.0,19.9,38.3,44.4$, 73.2, 110.0, 126.0, 127.6, 128.4, 144.9, 150.3; HRMS calcd for $\mathrm{C}_{13} \mathrm{H}_{18} \mathrm{O}$ : 190.1358, found $m / z \quad$ (relative intensity) $190\left(\mathrm{M}^{+}, 17\right), 172$ (10), 121 (35), 107 (100), 91 (10), 77 (35). Anal. calcd for $\mathrm{C}_{13} \mathrm{H}_{18} \mathrm{O}: \mathrm{C}, 82.06 ; \mathrm{H}, 9.53$, found $\mathrm{C}, 81.93 ; \mathrm{H}, 9.59$.

(4E)-1,2-anti-2-Methoxycarbonyl-1-phenyl-4-hexenol (2.12): IR (neat) 3450 (s), 2950 
(m), $1730(\mathrm{~s}), 1450(\mathrm{~m}), 1350$ (m), 1220 (m), 1160 (s), $1020(\mathrm{~m}), 960(\mathrm{~m}), 690(\mathrm{~s}) \mathrm{cm}^{-1} ;{ }^{1} \mathrm{H}$ NMR (400 MHz, $\left.\mathrm{CDCl}_{3}\right) \delta 1.60(\mathrm{br} \mathrm{d}, J=6.2 \mathrm{~Hz}, 3 \mathrm{H}), 2.32(\mathrm{~m}, 1 \mathrm{H}$, coalescing to ddd, $J=$ $4.8,7.3,14.1 \mathrm{~Hz}$ by irradiation at 1.60$), 2.41(\mathrm{~m}, 1 \mathrm{H}$, coalescing to ddd, $J=6.6,10.1,14.1$ $\mathrm{Hz}$ by irradiation at 1.60), $2.78(\mathrm{ddd}, J=4.8,5.7,10.1 \mathrm{~Hz}, 1 \mathrm{H}), 2.84(\mathrm{~d}, J=2.9 \mathrm{~Hz}, 1 \mathrm{H})$, 3.57 (br s, $3 \mathrm{H}), 4.97$ (dd, $J=2.9,5.7 \mathrm{~Hz}, 1 \mathrm{H}), 5.33$ (m, $1 \mathrm{H}$, coalescing to ddd, $J=6.6,7.3$, $15.0 \mathrm{~Hz}$ by irradiation at 1.60$), 5.45(\mathrm{~m}, 1 \mathrm{H}$, coalescing to $\mathrm{d}, J=15.0 \mathrm{~Hz}$ by irradiation at 1.60), 7.22-7.37 (m, $5 \mathrm{H}) ;{ }^{13} \mathrm{C} \mathrm{NMR}\left(100 \mathrm{MHz}, \mathrm{CDCl}_{3}\right) \delta$ 17.9, 30.4, 51.5, 53.2, 73.9, 126.1, 127.5, 127.6, 127.7, 128.3, 141.5, 174.8; HRMS, calcd for $\mathrm{C}_{14} \mathrm{H}_{18} \mathrm{O}_{3}$ : 234.1256, found $\mathrm{m} / \mathrm{z}$ (relative intensity) $234.1260\left(\mathrm{M}^{+}, 18\right), 216(58), 179$ (22), $157(40), 128$ (100), 77 (18). Anal calcd for $\mathrm{C}_{14} \mathrm{H}_{18} \mathrm{O}_{3}$ : C, 71.77; H, 7.74, found: $\mathrm{C}, 71.45 ; \mathrm{H}, 7.81$.

(4E)-1,2-anti-1-Phenyl-2-Methoxymethyl-4-hexen-1-ol (2.13): IR (neat) 3430 (s), 3060 (w), 3020 (m), 2920 (s), 2890 (s), 2820 (m), 1680 (m), 1600 (w), 1450 (s), 1380 (m), 1190 (m), 1110 (s), 371020 (m), 960 (s), 750 (m), 730 (m), 700 (s) cm ${ }^{-1}$; ${ }^{1} \mathrm{H}$ NMR (400 MHz, $\left.\mathrm{CDCl}_{3}\right) \delta 1.63(\mathrm{dd}, J=1.5,6.2 \mathrm{~Hz}, 3 \mathrm{H}), 1.99(\mathrm{~m}, 1 \mathrm{H}), 2.01(\mathrm{~m}, 2 \mathrm{H}), 3.34(\mathrm{~s}, 3 \mathrm{H})$, 3.38-3.45 (m, $2 \mathrm{H}), 4.93(\mathrm{dd}, J=4.0,4.8 \mathrm{~Hz}, 1 \mathrm{H}), 5.31$ (ddt, $J=1.5,15.2,6.6 \mathrm{~Hz}, 1 \mathrm{H})$, $5.43(\mathrm{dt}, J=15.2,6.2 \mathrm{~Hz}, 1 \mathrm{H}), 7.32(\mathrm{~m}, 5 \mathrm{H})$; HRMS, calcd for $\mathrm{C}_{14} \mathrm{H}_{20} \mathrm{O}_{2}: 220.1463$, found $m / z$ (relative intensity) $220.1450\left(\mathrm{M}^{+}, 9\right), 173(54), 157$ (31), 107 (93), 82 (100), 77 (45).

5-(trans-But-2-enyl)-2-ethyl-4-phenyl-[1,3,2]dioxaborinane (2.14): IR (neat) 3020 (w), 2950 (s), 2930 (s), 2900 (s), 2870 (s), 1480 (s), 1450 (s), 1430 (s), 1410 (s), 1370 (s), 1330 (s), 1270 (s), 1210 (s), 1070 (w), 1030 (m), 960 (s), 930 (w), 750 (s), 690 (s) cm-1; ${ }^{1} \mathrm{H}$ NMR $\left(400 \mathrm{MHz}, \mathrm{CDCl}_{3}\right) \delta 0.82(\mathrm{q}, J=7.7 \mathrm{~Hz}, 3 \mathrm{H}), 0.98(\mathrm{t}, J=7.7 \mathrm{~Hz}, 3 \mathrm{H}), 1.61$ (br d, $J$ $=6.1 \mathrm{~Hz}, 3 \mathrm{H}), 1.70(\mathrm{~m}, 2 \mathrm{H}), 2.17(\mathrm{~m}, 1 \mathrm{H}), 3.83(\mathrm{dd}, J=6.2,11.4 \mathrm{~Hz}, 1 \mathrm{H}), 4.01(\mathrm{dd}, J=$ 3.8, 11.4 Hz, $1 \mathrm{H}), 5.21$ (d, $J=4.0 \mathrm{~Hz}, 1 \mathrm{H}), 5.26$ (ddt, $J=7.7,15.0,1.5 \mathrm{~Hz}, 1 \mathrm{H}), 5.38$ (dq, $J=15.0,6.1 \mathrm{~Hz}, 1 \mathrm{H}), 7.23-7.38(\mathrm{~m}, 5 \mathrm{H})$; HRMS, calcd for $\mathrm{C}_{15} \mathrm{H}_{21} \mathrm{O}_{2} \mathrm{~B}: 244.1635$, found 
$m / z \quad$ (relative intensity) 244.1612 (M+, 100), 201 (82), 188 (70), 107 (29), 77 (9).

4-Cyclohexylidene-1-phenylbutan-1-ol (2.15): IR (neat) 3350 (s), 2910 (s), 1720 (m), 1600 (w), 1490 (s), 1440 (s), 1240 (s), 1040 (s), 900 (m), 830 (m), 740 (s), 680 (s) cm ${ }^{-1} ;{ }^{1} \mathrm{H}$ NMR (400 MHz, $\left.\mathrm{CDCl}_{3}\right) \delta 1.47-1.57(\mathrm{~m}, 7 \mathrm{H}), 1.71-1.78(\mathrm{~m}, 1 \mathrm{H}), 1.79-1.88(\mathrm{~m}, 1 \mathrm{H})$, 2.04-2.12 (m, $6 \mathrm{H}), 4.69(\mathrm{dd}, J=5.3,7.9 \mathrm{~Hz}, 1 \mathrm{H}), 5.10$ (br t, $J=7.3 \mathrm{~Hz}, 1 \mathrm{H})$; HRMS, calcd for $\mathrm{C}_{16} \mathrm{H}_{22} \mathrm{O}: 230.1671$, found $m / z$ (relative intensity) $230.1672\left(\mathrm{M}^{+}, 45\right), 213\left(\mathrm{M}^{+}-\right.$ OH, 11), 133 (45), 120 (45), 108 (100), 107 (43).

1,3-anti-3-Triisopropylsilyloxy-1-phenyl-4-pentenol (2.16): IR (neat) 3435 (s), 2935 (s), 2860 (s), 1695 (s), 1600 (w), 1460 (s), 1200 (m), 1060 (m), 990 (m), 740 (m), 670 (s) cm-1; ${ }^{1} \mathrm{H}$ NMR (400 MHz, $\left.\mathrm{CDCl}_{3}\right) \delta 1.06-1.18(\mathrm{~m}, 21 \mathrm{H}), 1.81(\mathrm{ddd}, J=2.6,4.2,14.5 \mathrm{~Hz}, 1 \mathrm{H})$, $2.12(\mathrm{ddd}, J=4.2,10.4,14.5 \mathrm{~Hz}, 1 \mathrm{H}), 4.00$ (br s, $1 \mathrm{H}), 4.66$ (br dt, $J=5.9,4.2 \mathrm{~Hz}, 1 \mathrm{H})$, $5.04(\mathrm{dd}, J=2.6,10.4 \mathrm{~Hz}, 1 \mathrm{H}), 5.24(\mathrm{br} \mathrm{dd}, J=1.5,10.6 \mathrm{~Hz}, 1 \mathrm{H}), 5.35$ (br dd, $J=1.5$, $17.2 \mathrm{~Hz}, 1 \mathrm{H}), 6.03$ (ddd, $J=5.9,10.6,17.2 \mathrm{~Hz}, 1 \mathrm{H}), 7.21-7.39(\mathrm{~m}, 5 \mathrm{H}) ;{ }^{13} \mathrm{C}$ NMR $(100$ $\left.\mathrm{MHz}, \mathrm{CDCl}_{3}\right) \delta 12.3,18.1,45.9,71.0,73.4,115.1,125.7,127.2,128.3,139.9,144.9$. Anal. calcd for $\mathrm{C}_{20} \mathrm{H}_{34} \mathrm{O}_{2} \mathrm{Si}: \mathrm{C}, 71.80, \mathrm{H}, 10.24$, found $\mathrm{C}, 71.85, \mathrm{H}, 10.34$.

1,3-anti-4-Methyl-1-phenyl-3-triisopropylsiloxy-4-pentenol (2.17): IR (neat ) 3410 (s), 2860 (s), 1470 (s), 1380 (m), 1250 (m), 1060 (s), 990 (s), 880 (s), 800 (s), 660 (s) cm-1 ; 1H NMR (400 MHz,CDCl3), $\delta 1.05-1.11$ (m, $21 \mathrm{H}), 1.76$ (s, $3 \mathrm{H}), 1.89$ (ddd, $J=2.4,3.7$, 14.7 Hz, $1 \mathrm{H}), 2.07$ (ddd, $J=4.8,10.3,14.7 \mathrm{~Hz}, 1 \mathrm{H}), 3.87$ (s, 1H), 4.50 (br dd, $J=3.7,4.8$ Hz, 1H), 4.91 (dd, $J=2.4,10.3 \mathrm{~Hz}, 1 \mathrm{H}), 5.04$ (s, $1 \mathrm{H}), 5.23$ (s, $1 \mathrm{H}), 7.26-7.34$ (m, $5 \mathrm{H})$; HRMS calcd for $\mathrm{C}_{21} \mathrm{H}_{36} \mathrm{O}_{2} \mathrm{Si}-\mathrm{C}_{3} \mathrm{H}_{7}: 305.1937$, found $m / z$ (relative intensity) 305.1950 $\left(\mathrm{M}^{+}-\mathrm{C}_{3} \mathrm{H}_{7}, 6\right), 227(2), 201(100), 131$ (25).

(4E)-1,3-anti-1,5-Diphenyl-3-triisopropylsiloxy-4-pentenol (2.18): IR (neat ) 3500 (s), 2940 (s), 1670 (s), 1610 (s), 1460 (m), 1360 (m), 1250 (s), 1050 (m), 970 (s), 880 (s), 830 (m), 740 (s), 680 (s) cm-1; 1H NMR (400 MHz,CDCl3) $\delta 1.11-1.14$ (m, $21 \mathrm{H}), 1.92$ (ddd, $J$ 
= 2.4, 4.4, $14.7 \mathrm{~Hz}, 1 \mathrm{H}), 2.19(\mathrm{ddd}, J=4.4,10.3,14.7 \mathrm{~Hz}, 1 \mathrm{H}), 4.00(\mathrm{~s}, 1 \mathrm{H}), 4.81(\mathrm{~m}, 1$ H), $5.09(\mathrm{dd}, J=2.4,10.3 \mathrm{~Hz}, 1 \mathrm{H}), 6.39(\mathrm{dd}, J=6.4,16.1 \mathrm{~Hz}, 1 \mathrm{H}), 6.63(\mathrm{~d}, J=16.1 \mathrm{~Hz}, 1$ H), 7.24-7.42 39 (m, $10 \mathrm{H}$ ); HRMS calcd for $\mathrm{C}_{26} \mathrm{H}_{38} \mathrm{O}_{2} \mathrm{Si}$ : 410.2641, found $\mathrm{m} / z$ (relative intensity) $410.2663\left(\mathrm{M}^{+},<1\right), 392$ (1), 289 (3), 263 (100).

(4E)-1-Phenyl-5-triisopropylsilyloxy-4-pentenol (2.19): IR (neat) 3360 (s), 3020 (w), 2930 (s), 2860 (s), 1660 (s), 1460 (s), 1380 (m), 1250 (m), 1180 (s), 1150 (s), 1050 (s), 1010 (s), 990 (s), 910 (s), 880 (s), 790 (s), 750 (s), 670 (s) cm-1; ${ }^{1} \mathrm{H}$ NMR (400 MHz, $\left.\mathrm{CDCl}_{3}\right), \delta 1.02-1.10(\mathrm{~m}, 21 \mathrm{H}), 1.60($ br s, $1 \mathrm{H}), 1.68-2.19(\mathrm{~m}, 4 \mathrm{H}), 4.70(\mathrm{dd}, J=5.3,7.9$ Hz, $1 \mathrm{H}), 5.02$ (dt, $J=11.7,7.3 \mathrm{~Hz}, 1 \mathrm{H}), 6.34$ (br d, $J=11.7 \mathrm{~Hz}, 1 \mathrm{H}), 7.26-7.38(\mathrm{~m}, 5 \mathrm{H})$; HRMS calcd for $\mathrm{C}_{20} \mathrm{H}_{34} \mathrm{O}_{2} \mathrm{Si}$ : 334.2328, found $\mathrm{m} / z$ (relative intensity) $334.2404\left(\mathrm{M}^{+}, 3\right.$ ), 291 (100), $275(69,159(11), 131$ (78).

(4E)-1,3-anti-3-Methyl-1-phenyl-5-trimethylsiloxy-4-pentenol (2.20): IR (neat ) 3370 (s), 3050 (w), 2950 (s), 2850 (s), 1660 (m), 1460 (m), 1380 (m), 1170 (w), 1060 (w) cm-1; ${ }^{1} \mathrm{H}$ NMR (400 MHz, $\left.\mathrm{CDCl}_{3}\right), \delta 0.94(\mathrm{~d}, J=6.6 \mathrm{~Hz}, 3 \mathrm{H}), 1.05-1.09(\mathrm{~m}, 21 \mathrm{H}), 1.71-1.81(\mathrm{~m}, 1$ H), 2.02-2.27 (m, $2 \mathrm{H}), 4.72(\mathrm{t}, J=7.0 \mathrm{~Hz}, 1 \mathrm{H}), 4.95(\mathrm{dd}, J=9.3,12.1 \mathrm{~Hz}, 1 \mathrm{H}), 6.32(\mathrm{~d}, J$ $=12.1 \mathrm{~Hz}, 1 \mathrm{H}$ ); HRMS, calcd for $\mathrm{C}_{21} \mathrm{H}_{36} \mathrm{O}_{2} \mathrm{Si}$ : 348.2485, found $\mathrm{m} / z$ (relative intensity) $348.2491\left(\mathrm{M}^{+}, 1\right), 305(87), 131$ (100), $107(10), 68(2)$.

(4E)-1,3-anti-5-Methoxy-1-phenyl-3-trimethylsiloxy-4-pentenol (2.21): IR (neat) 3422 (s), 2909 (s), 1655 (s), 1603 (w), 1493 (m), 1454 (s), 1211 (s), 843 (s), 758 (s), 702 (s) $\mathrm{cm}^{-1} ;{ }^{1} \mathrm{H}$ NMR $\left(400 \mathrm{MHz}, \mathrm{CDCl}_{3}\right) \delta 0.13(\mathrm{~s}, 9 \mathrm{H}), 1.94(\mathrm{~m}, 2 \mathrm{H}), 3.54(\mathrm{~s}, 3 \mathrm{H}), 3.55(\mathrm{~d}, J=$ $3.8 \mathrm{~Hz}, 1 \mathrm{H}), 4.36(\mathrm{~m}, 1 \mathrm{H}$, coalescing to $\mathrm{d}, J=8.4 \mathrm{~Hz}$, by irradiation at 1.94$), 4.89(\mathrm{dd}, J=$ 8.4, $12.8 \mathrm{~Hz}, 1 \mathrm{H}), 4.99$ ( $\mathrm{m}, 1 \mathrm{H}$, coalescing to $\mathrm{d}, J=3.8 \mathrm{~Hz}$, by irradiation at 1.94), 6.45 (d, $J=12.8 \mathrm{~Hz}, 1 \mathrm{H}), 7.25-7.35(\mathrm{~m}, 5 \mathrm{H}) ;{ }^{13} \mathrm{C} \mathrm{NMR}\left(100 \mathrm{MHz}, \mathrm{CDCl}_{3}\right), \delta 0.5,47.1,56.0$, 69.6, 71.3, 105.6, 125.7, 127.1, 128.6, 144.8, 149.0; HRMS calcd for $\mathrm{C}_{15} \mathrm{H}_{24} \mathrm{O}_{3} \mathrm{Si}$ : 280.1495, found $m / z$ (relative intensity) $280.1484\left(\mathrm{M}^{+}, 3\right), 262$ (24), 248 (100). 
2-Ethyl-4-(2-methyl-3-butenyl)-4H-benzo[1,3,2]dioxaborinine (2.22): IR (neat) 2881 (s), 1591 (s), 1492 (s), 1460 (s), 1402 (s), 1344 (s), 1240 (s), 1205 (s), 914 (m), 758 (s) cm-1; ${ }^{1} \mathrm{H}$ NMR (400 MHz, $\left.\mathrm{CDCl}_{3}\right) \delta 0.89$ (br q, $\left.J=8.2 \mathrm{~Hz}, 2 \mathrm{H}\right), 1.00$ (br t, $J=8.2 \mathrm{~Hz}, 3 \mathrm{H}$ ), $1.10(\mathrm{~d}, J=7.0 \mathrm{~Hz}, 3 \mathrm{H}), 1.64(\mathrm{ddd}, J=4.6,8.2,13.9 \mathrm{~Hz}, 1 \mathrm{H}), 1.86(\mathrm{ddd}, J=5.7,8.8,13.9$ $\mathrm{Hz}, 1 \mathrm{H}), 2.52$ (m, $1 \mathrm{H}), 4.93$ (br d, $J=10.3 \mathrm{~Hz}, 1 \mathrm{H}), 5.01$ (br d, $J=17.2 \mathrm{~Hz}, 1 \mathrm{H}), 5.08$ $(\mathrm{dd}, J=4.6,8.8 \mathrm{~Hz}, 1 \mathrm{H}), 5.78(\mathrm{ddd}, J=7.3,10.3,17.2 \mathrm{~Hz}, 1 \mathrm{H}), 6.91-7.20$ (m, $4 \mathrm{H})$; HRMS calcd for $\mathrm{C}_{14} \mathrm{H}_{19} \mathrm{BO}_{2}: 230.1478$, found $m / z$ (relative intensity) $230.1452\left(\mathrm{M}^{+}, 100\right)$, 229 (26).

1,3-anti-1-(m-Hydroxyphenyl)-3-methyl-4-penten-1-ol $\quad(\mathbf{2 . 2 3}): \mathrm{mp}=77.5-78.5{ }^{\circ} \mathrm{C}$ ( $\mathrm{CH}_{2} \mathrm{Cl}_{2}$ /hexane); IR (KBr) 3203 (s), 2957 (s), 1595 (s), 1472 (s), 1273 (s), 997 (s), 908 (s), $781(\mathrm{~s}), 696(\mathrm{~s}) \mathrm{cm}^{-1} ;{ }^{1} \mathrm{H} \mathrm{NMR}\left(400 \mathrm{MHz}, \mathrm{CDCl}_{3}\right) \delta 1.02(\mathrm{~d}, J=6.6 \mathrm{~Hz}, 3 \mathrm{H}), 1.63(\mathrm{dt}, J=$ 13.6, 6.2 Hz, $1 \mathrm{H}), 1.83$ (dt, $J=13.6,7.7 \mathrm{~Hz}, 1 \mathrm{H}), 2.13$ (br s, $1 \mathrm{H}), 2.22(\mathrm{~m}, 1 \mathrm{H}), 4.69$ (m, $1 \mathrm{H}), 4.97$ (br d, $J=10.3 \mathrm{~Hz}, 1 \mathrm{H}), 5.00$ (br d, $J=17.2 \mathrm{~Hz}, 1 \mathrm{H}), 5.39$ (s, $1 \mathrm{H}), 5.77$ (ddd, $J$ $=7.3,10.3,17.2 \mathrm{~Hz}, 1 \mathrm{H}), 6.74(\mathrm{dd}, J=2.6,7.9 \mathrm{~Hz}, 1 \mathrm{H}), 6.85(\mathrm{~d}, J=2.6 \mathrm{~Hz}, 1 \mathrm{H}), 6.87(\mathrm{~d}$, $J=7.9 \mathrm{~Hz}, 1 \mathrm{H}), 7.19(\mathrm{t}, J=7.9 \mathrm{~Hz}, 1 \mathrm{H}) ;{ }^{13} \mathrm{C} \mathrm{NMR}\left(100 \mathrm{MHz}, \mathrm{CDCl}_{3}\right) \delta 20.5,35.1,45.8$, 73.0, 112.9, 113.3, 114.7, 118.5, 129.8, 144.5, 146.6, 156.0; HRMS calcd for $\mathrm{C}_{12} \mathrm{H}_{16} \mathrm{O}_{2}$ : 192.1150, found $\mathrm{m} / \mathrm{z}$ (relative intensity) $192.1144\left(\mathrm{M}^{+}, 9\right), 174(1), 123(100)$.

1,3-anti-1-(p-Hydroxyphenyl)-3-methyl-4-penten-1-ol (2.24): $\mathrm{mp}=133.5-134.5^{\circ} \mathrm{C}$; IR (KBr) 3352 (s), 1616 (s), 1600 (s), 1515 (s), 1460 (s), 1375 (m), 1238 (s), 1178 (m), 985 (s), $916(\mathrm{~m}), 839(\mathrm{~s}) \mathrm{cm}^{-1} ;{ }^{1} \mathrm{H} \mathrm{NMR}\left(400 \mathrm{MHz}, \mathrm{CDCl}_{3}\right) \delta 1.01(\mathrm{~d}, J=6.6 \mathrm{~Hz}, 3 \mathrm{H}), 1.64(\mathrm{dt}, J$ $=13.7,6.6 \mathrm{~Hz}, 1 \mathrm{H}), 1.85(\mathrm{dt}, J=13.7,7.5 \mathrm{~Hz}, 1 \mathrm{H}), 1.92(\mathrm{br} \mathrm{s}, 1 \mathrm{H}), 2.17(\mathrm{~m}, 1 \mathrm{H}), 4.67$ (br dd, $J=6.6,7.5 \mathrm{~Hz}, 1 \mathrm{H}), 4.96$ (br d, $J=10.3 \mathrm{~Hz}, 1 \mathrm{H}), 4.98$ (br d, $J=17.2 \mathrm{~Hz}, 1 \mathrm{H}$ ), 5.14 (br s, $1 \mathrm{H}), 5.77$ (ddd, $J=7.7,10.3,17.2 \mathrm{~Hz}, 1 \mathrm{H}), 6.79$ (d, $J=8.6 \mathrm{~Hz}, 2 \mathrm{H}), 7.21$ (d, $J$ $=8.6 \mathrm{~Hz}, 2 \mathrm{H}) ;{ }^{13} \mathrm{C} \mathrm{NMR}\left(100 \mathrm{MHz}, \mathrm{CDCl}_{3}\right) \delta 20.6,35.5,45.8,72.9,113.3,115.4,127.6$, 136.8, 144.6, 155.2; HRMS calcd for $\mathrm{C}_{12} \mathrm{H}_{16} \mathrm{O}_{2}$ : 192.1150, found $m / z$ (relative intensity) 
$192.1145\left(\mathrm{M}^{+}, 64\right), 175(14), 174(100)$.

1,3-anti-1-(2-Furyl)-3-metyl-4-penten-1-ol (2.25): IR (neat) 3350 (s), 3070 (w), 2960 (s), 2930 (s), 2860 (w), 1650 (w), 1510 (m), 1460 (m), 1420 (m), 1370 (m), 1230 (m), 1150 (s), 1070 (s), 1010 (s), 910 (s), 800 (s), 730 (s) cm-1; ${ }^{1} \mathrm{H}$ NMR (400 MHz, $\left.\mathrm{CDCl}_{3}\right) \delta 1.04$ (d, $J$ $=6.6 \mathrm{~Hz}, 3 \mathrm{H}), 1.83(\mathrm{dt}, J=6.8,13.5 \mathrm{~Hz}, 1 \mathrm{H}), 1.89(\mathrm{dt}, J=7.5,13.5,1 \mathrm{H}), 2.23(\mathrm{~m}, 1 \mathrm{H}$, coalescing to br dt, $J=6.8,7.5 \mathrm{~Hz}$ by irradiation at 1.04$), 4.74(\mathrm{br} \mathrm{d}, J=6.8,7.5 \mathrm{~Hz}, 1 \mathrm{H})$, 4.96 (br d, $J=10.3 \mathrm{~Hz}, 1 \mathrm{H}), 4.97$ (br d, $J=17.4 \mathrm{~Hz}, 1 \mathrm{H}$ ), 5.75 (ddd, $J=7.5,10.3,17.4 \mathrm{~Hz}$, $1 \mathrm{H}), 6.23(\mathrm{~d}, J=3.3 \mathrm{~Hz}, 1 \mathrm{H}), 6.33(\mathrm{dd}, J=1.6,3.3 \mathrm{~Hz}, 1 \mathrm{H}), 7.38(\mathrm{~d}, J=1.6 \mathrm{~Hz}, 1 \mathrm{H})$. Anal. calcd for $\mathrm{C}_{10} \mathrm{H}_{14} \mathrm{O}_{2}$ : C, 72.26; $\mathrm{H}, 8.49$, found: C, 72.12; H, 8.56.

(2E)-4,6-anti-6-Methyl-octa-2,7-dien-4-ol (2.26): IR (neat) 3340 (s), 2910 (s), 1650 (w), 1450 (m), 1370 (m), 990 (m), 960 (s), 905 (s) cm-1; ${ }^{1} \mathrm{H} \mathrm{NMR} \mathrm{(400} \mathrm{MHz,} \mathrm{CDCl}_{3}$ ) $\delta 1.02$ (d, $J=6.6 \mathrm{~Hz}, 3 \mathrm{H}), 1.41(\mathrm{dt}, J=6.2,13.4 \mathrm{~Hz}, 1 \mathrm{H}), 1.59(\mathrm{dt}, J=8.1,13.4 \mathrm{~Hz}, 1 \mathrm{H}), 1.70(\mathrm{dd}, J$ $=1.7,6.6 \mathrm{~Hz}, 3 \mathrm{H}), 2.25(\mathrm{~m}, 1 \mathrm{H}), 4.10(\mathrm{~m}, 1 \mathrm{H}), 4.93(\mathrm{br} \mathrm{dd}, J=0.9,10.3 \mathrm{~Hz}, 1 \mathrm{H}), 5.00$ (br dd, $J=0.9,17.2 \mathrm{~Hz}, 1 \mathrm{H}), 5.46(\mathrm{ddq}, J=7.3,15.2,1.7 \mathrm{~Hz}, 1 \mathrm{H}), 5.64(\mathrm{dq}, J=15.2,6.6$ $\mathrm{Hz}, 1 \mathrm{H}), 5.73(\mathrm{ddd}, J=7.7,10.3,17.2 \mathrm{~Hz}, 1 \mathrm{H}) ;{ }^{13} \mathrm{C} \mathrm{NMR}\left(100 \mathrm{MHz}, \mathrm{CDCl}_{3}\right) \delta 17.7,20.5$, 34.9, 44.2, 71.5, 112.9, 126.9, 134.3, 144.7; HRMS calcd for $\mathrm{C}_{9} \mathrm{H}_{16} \mathrm{O}: 140.1201$, found $\mathrm{m} / z$ (relative intensity) $140.1227\left(\mathrm{M}^{+}, 22\right), 139$ (100), $122(64)$.

(1E)-3,5-anti-5-Methyl-1-phenyl-1,6-heptadien-3-ol (2.27): IR (neat) 3350 (s), 3060 (w), 3010 (w), 2950 (s), 2920 (s), 2860 (w), 1640 (w), 1490 (m), 1450 (m), 1370 (s), 1330 (s), 1220 (m), 1060 (m), 990 (s), 960 (s), 910 (s), 740 (s), 690 (s) cm-1; ${ }^{1} \mathrm{H}$ NMR (400 MHz, $\left.\mathrm{CDCl}_{3}\right) \delta 1.05(\mathrm{~d}, J=6.6 \mathrm{~Hz}, 3 \mathrm{H}), 1.55(\mathrm{dt}, J=6.5,13.6 \mathrm{~Hz}, 1 \mathrm{H}), 1.71$ (ddd, $J=7.1,8.3$, 13.6 Hz, $1 \mathrm{H}), 2.34(\mathrm{~m}, 1 \mathrm{H}$, coalescing to br dt, $J=6.6,8.3 \mathrm{~Hz}$ by irradiation at 1.05$), 4.35$ (br dt, $J=6.5,7.1 \mathrm{~Hz}, 1 \mathrm{H}), 4.97$ (br d, $J=10.2 \mathrm{~Hz}, 1 \mathrm{H}$ ), 5.03 (br d, $J=17.2 \mathrm{~Hz}, 1 \mathrm{H}$ ), $5.77(\mathrm{ddd}, J=8.3,10.2,17.2 \mathrm{~Hz}, 1 \mathrm{H}), 6.20(\mathrm{dd}, J=7.1,15.9 \mathrm{~Hz}, 1 \mathrm{H}), 6.56(\mathrm{~d}, J=15.9 \mathrm{~Hz}$, $1 \mathrm{H}), 7.21-7.41(\mathrm{~m}, 5 \mathrm{H}) ;{ }^{13} \mathrm{C} \mathrm{NMR}\left(100 \mathrm{MHz} \mathrm{CDCl}_{3}\right) \delta 20.5,35.0,44.2,71.5,113.1$, 
126.5, 127.7, 128.6, 130.4, 132.5, 136.8, 144.5. Anal. calcd for $\mathrm{C}_{14} \mathrm{H}_{18} \mathrm{O}: \mathrm{C}, 83.12 ; \mathrm{H}$, 8.97, found: C, 82.74; H, 9.16.

4,6-anti-2,6-Dimethyl-2,7-octadien-4-ol (2.28): IR (neat) 3330(s), 2910 (s), 1670 (m), 1650 (s), 1440 (s), 1370 (s), 1290 (s), 990 (s), 900 (s), 830 (m) cm-1' ; ${ }^{1} \mathrm{H}$ NMR (400 MHz, $\left.\mathrm{CDCl}_{3}\right), \delta 1.02(\mathrm{~d}, J=6.6 \mathrm{~Hz}, 3 \mathrm{H}), 1.38(\mathrm{ddd}, J=5.9,6.8,13.6 \mathrm{~Hz}, 3 \mathrm{H}), 1.63$ (ddd, $J=$ 6.8, 8.8, 13.6 Hz, $1 \mathrm{H}), 1.67$ (d, $J=1.5 \mathrm{~Hz}, 3 \mathrm{H}), 1.73(\mathrm{~d}, J=1.5 \mathrm{~Hz}, 3 \mathrm{H}), 2.20(\mathrm{~m}, 1 \mathrm{H})$, 4.39 (dt, $J=6.8,8.8 \mathrm{~Hz}, 1 \mathrm{H}), 4.92(\mathrm{br} \mathrm{d}, J=10.3 \mathrm{~Hz}, 1 \mathrm{H}), 4.96(\mathrm{br} \mathrm{d}, J=17.2 \mathrm{~Hz}, 1 \mathrm{H})$, $5.15(\mathrm{dt}, J=8.8,1.5 \mathrm{~Hz}, 1 \mathrm{H}), 5.74(\mathrm{ddd}, J=8.1,10.3,17.2 \mathrm{~Hz}, 1 \mathrm{H})$; ; HRMS, calcd for $\mathrm{C}_{10} \mathrm{H}_{18} \mathrm{O}: 154.1358$, found $\mathrm{m} / z$ (relative intensity) $154.1360\left(\mathrm{M}^{+}, 26\right), 139$ (100), 137 (16), $136(65)$.

(2E)-4,6-anti-3,6-Dimethyl-2,7-octadien-4-ol (2.29): IR (neat) 3350 (s), 2940 (s), 1650 (m), 1450 (s), 1380 (s), 1340 (m), 1040 (m), 1000 (s), 910 (s), 830 (m) cm-1; ${ }^{1} \mathrm{H}$ NMR (400 $\left.\mathrm{MHz}, \mathrm{CDCl}_{3}\right) \delta 1.02(\mathrm{~d}, J=7.0 \mathrm{~Hz}, 1 \mathrm{H}), 1.44(\mathrm{~d}, J=3.3 \mathrm{~Hz}, 1 \mathrm{H}), 1.47(\mathrm{dt}, J=13.5,6.2$ $\mathrm{Hz}, 1 \mathrm{H}), 1.57(\mathrm{dm}, J=13.5 \mathrm{~Hz}, 1 \mathrm{H}), 1.58(\mathrm{~s}, 3 \mathrm{H}), 1.61$ (s, $3 \mathrm{H}), 2.18$ (m, $1 \mathrm{H}), 4.07$ (m, 1 H), $4.93(\mathrm{dd}, J=1.1,10.3 \mathrm{~Hz}, 1 \mathrm{H}), 4.98(\mathrm{dd}, \mathrm{J}=1.1,17.2 \mathrm{~Hz}, 1 \mathrm{H}), 5.74$ (ddd, $\mathrm{J}=7.7$, 10.3, 17.2 Hz, $1 \mathrm{H}$ ); HRMS, calcd for $\mathrm{C}_{10} \mathrm{H}_{18} \mathrm{O}-\mathrm{H}_{2}: 152.1199$, found $\mathrm{m} / z$ (relative intensity) $152.1241\left(\mathrm{M}^{+}-\mathrm{H}_{2}, 3\right), 139\left(\mathrm{M}^{+}-\mathrm{CH}_{3}, 3\right), 121$ (2), 85 (2), 71 (100).

3,5-anti-5-Methyl-2-triisopropylsiloxy-1,6-heptadien-3-ol (2.30): IR (neat) 3402 (s), 2945 (s), 1631 (m), 1464 (m), 1256 (m), 1016 (s), 912 (m), 883 (s) cm ${ }^{-1} ;{ }^{1} \mathrm{H}$ NMR (400 $\left.\mathrm{MHz}, \mathrm{CDCl}_{3}\right) \delta 1.03(\mathrm{~d}, J=6.6 \mathrm{~Hz}, 3 \mathrm{H}), 1.10(\mathrm{~d}, J=6.6 \mathrm{~Hz}, 18 \mathrm{H}), 1.25(\mathrm{~m}, 3 \mathrm{H}), 1.58(\mathrm{dt}$, $J=13.5,7.0 \mathrm{~Hz}, 1 \mathrm{H}), 1.66(\mathrm{dt}, J=13.5,7.0 \mathrm{~Hz}, 1 \mathrm{H}), 1.88(\mathrm{~d}, J=7.0 \mathrm{~Hz}, 1 \mathrm{H}), 2.35(\mathrm{~m}, 1$ H), 3.98 (q, $J=7.0 \mathrm{~Hz}, 1 \mathrm{H}), 4.12(\mathrm{~d}, J=1.7 \mathrm{~Hz}, 1 \mathrm{H}), 4.23(\mathrm{~d}, J=1.7 \mathrm{~Hz}, 1 \mathrm{H}), 4.97$ (br d, $J=10.3 \mathrm{~Hz}, 1 \mathrm{H}), 5.01(\mathrm{br} \mathrm{d}, J=17.2 \mathrm{~Hz}, 1 \mathrm{H}), 5.76(\mathrm{ddd}, J=7.3,10.3,17.2 \mathrm{~Hz}, 1 \mathrm{H})$; HRMS calcd for $\mathrm{C}_{17} \mathrm{H}_{34} \mathrm{O}_{2} \mathrm{Si}$, found $\mathrm{m} / z$ (relative intensity) $298.2329\left(\mathrm{M}^{+}, 11\right), 255$ (100), $227(10)$. 
3,5-anti-3-Methyl-1decene-5-ol (2.31): IR (neat) 3350 (s), 3100 (w), 2950 (s), 1660 (m), 1470 (s), 1380 (m), 1140 (m), 1000 (s), 920 (s), 680 (m) cm-1; ${ }^{1} \mathrm{H}$ NMR (400 MHz, $\left.\mathrm{CDCl}_{3}\right) \delta 0.89(\mathrm{t}, J=6.7 \mathrm{~Hz}, 3 \mathrm{H}), 1.01(\mathrm{~d}, J=7.0 \mathrm{~Hz}, 3 \mathrm{H}), 1.29-1.52(\mathrm{~m}, 11 \mathrm{H}), 2.33(\mathrm{~m}$, $1 \mathrm{H}), 3.67(\mathrm{~m}, 1 \mathrm{H}), 4.93(\mathrm{~d}, J=10.3 \mathrm{~Hz}, 1 \mathrm{H}), 5.02$ (d, $J=17.4 \mathrm{~Hz}, 1 \mathrm{H}), 5.78$ (ddd, $J=8.1$, 10.3, $17.4 \mathrm{~Hz}, 1 \mathrm{H}$ ); HRMS calcd for $\mathrm{C}_{11} \mathrm{H}_{22} \mathrm{O}$ : 170.1671 , found $m / z$ (relative intensity) $170.1636\left(\mathrm{M}^{+}, 13\right), 169$ (100), 168 (24), 155 (5), 152 (4).

3,5-anti-3,7,11-Trimethyl-1,10-dodecadiene-5-ol (2.32): $1: 1$ diastereomeric mixture regarding C7 stereocenter, IR (neat) 3330 (s), 2920 (s), 1640 (m), 1450 (s), 1370 (s), 1110 (m), 1020 (m), 990 (s), 900 (s), 660 (m) cm-1; ${ }^{1} \mathrm{H}$ NMR (400 MHz, $\left.\mathrm{CDCl}_{3}\right) \delta 0.92$ (d, J= $6.6 \mathrm{~Hz}, 3 \mathrm{H}), 1.02(\mathrm{~d}, J=6.6 \mathrm{~Hz}, 3 \mathrm{H}), 1.11-1.66(\mathrm{~m}, 8 \mathrm{H}), 1.60$ (s, $3 \mathrm{H}), 1.68(\mathrm{~s}, 3 \mathrm{H}), 1.91$ (m, $2 \mathrm{H}), 2.33(\mathrm{~m}, 1 \mathrm{H}), 3.78(\mathrm{~m}, 1 \mathrm{H}), 4.94$ (br d, $J=10.3 \mathrm{~Hz}, 1 \mathrm{H}), 5.03$ (br d, $J=17.1 \mathrm{~Hz}$, $1 \mathrm{H}), 5.78$ (ddd, $J=6.2,10.3,17.1 \mathrm{~Hz}, 1 \mathrm{H}$ ); HRMS, calcd for $\mathrm{C}_{15} \mathrm{H}_{28} \mathrm{O}: 224.2140$, found $m / z$ (relative intensity) $224.2122\left(\mathrm{M}^{+}, 100\right), 209$ (9), 206 (21), 191 (14).

3,5-anti-5-Methyl-1-phenyl-6-hepten-3-ol (2.33): IR (neat) 3350 (s), 3100 (s), 3050 (s), 2930 (s), 2880 (s), 1650 (s), 1610 (s), 1500 (s), 1450 (s), 1420 (s), 1370 (s), 1090 (s), 1030 (s), $1000(\mathrm{~s}), 910(\mathrm{~s}), 860(\mathrm{~m}), 740(\mathrm{~s}), 690(\mathrm{~s}) \mathrm{cm}^{-1} ;{ }^{1} \mathrm{H} \mathrm{NMR}\left(400 \mathrm{MHz}, \mathrm{CDCl}_{3}\right) \delta 1.01(\mathrm{~d}$, $J=6.6 \mathrm{~Hz}, 3 \mathrm{H}), 1.43(\mathrm{ddd}, J=4.0,6.6,13.7 \mathrm{~Hz}, 1 \mathrm{H}), 1.53(\mathrm{dt}, J=13.7,7.8 \mathrm{~Hz}, 1 \mathrm{H}), 1.59$ (br s, $1 \mathrm{H}), 1.68-1.82(\mathrm{~m}, 2 \mathrm{H}), 2.32(\mathrm{~m}, 1 \mathrm{H}), 2.67$ (ddd, $J=7.0,9.5,13.6 \mathrm{~Hz}, 1 \mathrm{H}), 2.79$ $(\mathrm{ddd}, J=5.9,9.5,13.6 \mathrm{~Hz}, 1 \mathrm{H}), 3.72(\mathrm{~m}, 1 \mathrm{H}), 4.93($ br d, $J=10.3 \mathrm{~Hz}, 1 \mathrm{H}), 5.02$ (br d, $J=$ 17.4 Hz, $1 \mathrm{H}), 5.77$ (ddd, $J=8.1,10.3,17.4 \mathrm{~Hz}, 1 \mathrm{H}), 7.16-7.30(\mathrm{~m}, 5 \mathrm{H})$. Anal. calcd for $\mathrm{C}_{14} \mathrm{H}_{20} \mathrm{O}: \mathrm{C}, 82.30 ; \mathrm{H}, 9.87$, found: C, 82.16; H, 9.97.

3,5-anti-2-Benzyloxy-5-methyl-6-hepten-3-ol $\quad(\mathbf{2 . 3 4 )}: \quad 1: 1.2$ diastereomeric mixture regarding C2 stereocenter, IR (neat) 3430 (s), 3070 (m), 3040 (w), 2980 (m), 2940 (m), 1740 (w), 1650 (w), 1500 (w), 1450 (m), 1380 (m), 1070 (s) cm-1; ${ }^{1} \mathrm{H}$ NMR (400 MHz, $\left.\mathrm{CDCl}_{3}\right) \delta 1.01(\mathrm{~d}, J=6.6 \mathrm{~Hz}, 3 \mathrm{H}), 1.19(\mathrm{~d}, J=5.9 \mathrm{~Hz}, 3 \mathrm{H}), 1.51(\mathrm{dd}, J=5.5,9.5 \mathrm{~Hz}, 2 \mathrm{H})$, 
2.37-2.42 (m, $1 \mathrm{H}), 2.51$ (br s, $1 \mathrm{H}), 3.39$ (quin, $J=5.9 \mathrm{~Hz}, 1 \mathrm{H}$ ), 4.44 (d, $J=11.7 \mathrm{~Hz}, 1 \mathrm{H}$ ), $4.67(\mathrm{~d}, J=11.7 \mathrm{~Hz}, 1 \mathrm{H}), 4.92(\mathrm{br} \mathrm{d}, J=10.3 \mathrm{~Hz}, 1 \mathrm{H}), 4.99($ br d, $J=17.2 \mathrm{~Hz}, 1 \mathrm{H}), 5.80$ $(\mathrm{ddd}, J=7.0,10.3,17.2 \mathrm{~Hz}, 1 \mathrm{H}), 7.27-7.38(\mathrm{~m}, 5 \mathrm{H})$; HRMS calcd for $\mathrm{C}_{15} \mathrm{H}_{22} \mathrm{O}_{2}$ : 234.1620, found $m / z$ (relative intensity) $234.1629\left(\mathrm{M}^{+}, 100\right), 216$ (23). Anal. calcd for $\mathrm{C}_{15} \mathrm{H}_{22} \mathrm{O}_{2}$ : C, 76.88; H, 9.46; found, C, 76.90, H, 9.50.

3,5-anti-1-(2,2-Dimethyl-[1,3]dioxolane-4-yl)-3-methyl-4-pentenol (2.35): $1: 1.6$ diastereomeric mixture regarding C2 stereocenter, IR (neat) 3470 (s), 3060 (w), 2980 (m), 1650 (w), 1450 (m), 1370 (m), 1250 (m), 1210 (m), 1160 (s) cm ${ }^{-1}$; ${ }^{1} \mathrm{H}$ NMR (400 MHz, $\left.\mathrm{CDCl}_{3}\right) \delta 1.03(\mathrm{~d}, J=7.0 \mathrm{~Hz}, 3 \mathrm{H}), 1.37(\mathrm{~s}, 3 \mathrm{H}), 1.40-1.46(\mathrm{~m}, 4 \mathrm{H}), 1.59$ (ddd, $J=6.0$ 9.5, 13.9 Hz, 1 H), 2.05 (br d, $J=2.6 \mathrm{~Hz}, 1 \mathrm{H}), 2.41(\mathrm{~m}, 1 \mathrm{H}), 3.87(\mathrm{~m}, 1 \mathrm{H}), 3.92(\mathrm{t}, J=6.6$ $\mathrm{Hz}, 1 \mathrm{H}), 3.96$ (t, $J=6.6 \mathrm{~Hz}, 1 \mathrm{H}), 4.00(\mathrm{dt}, J=11.0,6.6 \mathrm{~Hz}, 1 \mathrm{H}), 4.95(\mathrm{br} \mathrm{d}, J=10.3 \mathrm{~Hz}$, $1 \mathrm{H}), 5.04$ (br d, $J=17.2 \mathrm{~Hz}, 1 \mathrm{H}), 5.79$ (ddd, $J=7.7,10.3,17.2 \mathrm{~Hz}, 1 \mathrm{H})$; HRMS calcd for $\mathrm{C}_{11} \mathrm{H}_{20} \mathrm{O}_{3}: 200.1412$, found $m / z$ (relative intensity) $200.1435\left(\mathrm{M}^{+}, 4\right), 185$ (100), 182 (1), 144 (7). Anal. calcd for $\mathrm{C}_{11} \mathrm{H}_{20} \mathrm{O}_{3}$ : C, 65.97; H, 10.07; found, C, 65.88, H, 9.98.

1,3-anti-1-Cyclohexyl-3-metyl-4-pentenol (2.36) : IR (neat) 3360 (s), 3090 (w), 2950 (s), $2860(\mathrm{~s}), 1650(\mathrm{w}), 1450(\mathrm{~m}), 1420(\mathrm{w}), 1380(\mathrm{w}), 1090(\mathrm{w}), 1060(\mathrm{w}), 1000(\mathrm{w}), 910(\mathrm{~m})$ $\mathrm{cm}^{-1} ;{ }^{1} \mathrm{H}$ NMR $\left(400 \mathrm{MHz}, \mathrm{CDCl}_{3}\right) \delta 1.01(\mathrm{~d}, J=6.6 \mathrm{~Hz}, 3 \mathrm{H}), 1.03-1.57(\mathrm{~m}, 12 \mathrm{H})$, 1.62-1.67 (m, $1 \mathrm{H}), 1.73-1.79(\mathrm{~m}, 1 \mathrm{H}), 2.34$ (m, $1 \mathrm{H}), 3.47$ (dt, J = 8.8, $4.4 \mathrm{~Hz}, 1 \mathrm{H}), 4.94$ (br d, $J=10.3 \mathrm{~Hz}, 1 \mathrm{H}), 5.04$ (br d, $J=17.4 \mathrm{~Hz}, 1 \mathrm{H}$ ), 5.81 (ddd, $J=7.7,10.3,17.4 \mathrm{~Hz}, 1$ $\mathrm{H}$ ); HRMS, calcd for $\mathrm{C}_{12} \mathrm{H}_{22} \mathrm{O}: 182.1671$, found $\mathrm{m} / z$ (relative intensity) $182.1655\left(\mathrm{M}^{+}\right.$, 12), 126 (38), 99 (72), 95 (100), 81 (81), 70 (97). Anal. calcd for $\mathrm{C}_{12} \mathrm{H}_{22} \mathrm{O}$ : C, 79.06; H, 12.16, found: C, 79.03; H, 12.22.

3,5-anti-2,2,5-Trimethyl-6-hepten-3-ol (2.37): IR (neat) 3402 (s), 2959 (s), 1639 (m), 1466 (m), 1366 (s), 1076 (s), 997 (s), 910 (s), 856 (m) cm-1; ${ }^{1} \mathrm{H}$ NMR (400 MHz, CDCl 3 ) $\delta$ 0.89 (s, $9 \mathrm{H}), 1.02$ (d, $J=6.6 \mathrm{~Hz}, 3 \mathrm{H}), 1.37$ (ddd, $J=5.9,9.4,13.9 \mathrm{~Hz}, 1 \mathrm{H}), 1.43$ (ddd, $J=$ 
2.6, 8.1, $13.9 \mathrm{~Hz}, 1 \mathrm{H}), 1.53(\mathrm{~d}, J=4.8 \mathrm{~Hz}, 1 \mathrm{H}), 2.35(\mathrm{~m}, 1 \mathrm{H}), 3.31$ (ddd, $J=2.6,4.8,9.4$ $\mathrm{Hz}, 1 \mathrm{H}), 4.94$ (br d, $J=10.3 \mathrm{~Hz}, 1 \mathrm{H}), 5.04$ (br d, $J=17.6 \mathrm{~Hz}, 1 \mathrm{H}), 5.84$ (ddd, $J=7.3$, 10.3, $17.6 \mathrm{~Hz}, 1 \mathrm{H}) ;{ }^{13} \mathrm{C} \mathrm{NMR}\left(100 \mathrm{MHz}, \mathrm{CDCl}_{3}\right) \delta 19.5,25.7,34.9,35.7,38.8,78.2$, 112.4, 145.8; HRMS calcd for $\mathrm{C}_{10} \mathrm{H}_{20} \mathrm{O}-\mathrm{H}_{2} \mathrm{O}: 139.1487$, found $\mathrm{m} / z$ (relative intensity) $139.1499\left(\mathrm{M}^{+}-\mathrm{OH}, 17\right), 138$ (100), 123 (69), 109 (28). Anal. calcd for $\mathrm{C}_{10} \mathrm{H}_{20} \mathrm{O}: \mathrm{C}$, 79.86; H, 12.90, found: C, 79.58; H, 13.01.

3,5-anti-2-(t-Butyldimethylsiloxy)-5-methyl-6-hepten-3-ol $\quad(\mathbf{2 . 3 8}): \quad 1: 1 \quad$ diastereomeric mixture regarding C2 stereocenter, IR (neat) 3450 (m), 3080 (w), 2950 (s), 2920 (s), 2850 (m), 1760 (w), 1650 (w), 1460 (m), 1370 (m), 1250 (s), 1070 (s) cm-1; ${ }^{1} \mathrm{H}$ NMR (400 MHz, $\left.\mathrm{CDCl}_{3}\right) \delta 0.06$ (s, $3 \mathrm{H}$, major), 0.07 (s, $3 \mathrm{H}$, major), 0.08 (s, $3 \mathrm{H}$, minor), 0.09 (s, $3 \mathrm{H}$, minor), 0.89 (s, $9 \mathrm{H}$, major), 0.90 (s, $9 \mathrm{H}$, minor), 1.01 (d, J=2.6 Hz, $3 \mathrm{H}$, minor), 1.03 (d, $J=2.2 \mathrm{~Hz}, 3 \mathrm{H}$, major), 1.07 (d, $J=6.6 \mathrm{~Hz}, 3 \mathrm{H}$, minor), 1.14 (d, $J=6.2 \mathrm{~Hz}, 3 \mathrm{H}$, major), 1.24-1.32 (m, $2 \mathrm{H}$, major + minor), 1.45-1.53 (m, $2 \mathrm{H}$, major + minor), $2.16(\mathrm{br} \mathrm{d}, J=2.9 \mathrm{H}$ z, 1H), 2.28-2.42 (m, $3 \mathrm{H}), 3.39(\mathrm{~m}, 1 \mathrm{H}), 3.61(\mathrm{~m}, 1 \mathrm{H}), 3.61(\mathrm{~m}, 1 \mathrm{H}$, minor) 3.64 (dt, $J=$ 6.2, 11.4 Hz, $1 \mathrm{H}$, minor), 3.77 (dq, $J=3.3,6.2 \mathrm{~Hz}, 1 \mathrm{H}$, major), 4.91 (br d, $J=10.6 \mathrm{~Hz}, 1$ H, minor), 4.93 (br d, $J=10.3 \mathrm{~Hz}, 1 \mathrm{H}$, major), 4.99 (br d, $J=17.2 \mathrm{~Hz}, 1 \mathrm{H}$, minor), 5.00 (br d, $J=17.2 \mathrm{~Hz}, 1 \mathrm{H}$, major), 5.97 (ddd, $J=7.0,10.6,17.2 \mathrm{~Hz}, 1 \mathrm{H}$, major), 5.80 (ddd, $J$ $=7.0,10.6,17.2 \mathrm{~Hz}, 1 \mathrm{H}$ ); HRMS, calcd for $\mathrm{C}_{14} \mathrm{H}_{30} \mathrm{O}_{2} \mathrm{Si}: 258.2015$, found $\mathrm{m} / z$ (relative intensity) $258.1935\left(\mathrm{M}^{+}, 25\right), 240$ (17), 225 (100). Anal. calcd for $\mathrm{C}_{14} \mathrm{H}_{30} \mathrm{O}_{2} \mathrm{Si}$ : C, 65.06; H, 11.70; found C, 65.18; H, 11.77 .

3,5-anti-2-Benzyloxy-5-(triisopropylsiloxy)-6-hepten-3-ol (2.39): $\quad 1: 1 \quad$ diastereomeric mixture regarding C2 stereocenter, IR (neat) 3473 (s), 2943 (s), 1069 (s), 679 (s) cm¹-1 ${ }^{1} \mathrm{H}$ NMR (400 MHz, $\mathrm{CDCl}_{3}$, one isomer) $\delta 1.05-1.12(\mathrm{~m}, 21 \mathrm{H}), 1.18(\mathrm{~d}, J=6.2 \mathrm{~Hz}, 3 \mathrm{H})$, $1.64(\mathrm{dm}, J=14.3 \mathrm{~Hz}, 1 \mathrm{H}), 1.70-1.80(\mathrm{~m}, 3 \mathrm{H}), 3.23$ (br d, $J=2.0 \mathrm{~Hz}, 1 \mathrm{H}), 3.44$ (qm, $J=$ $6.2 \mathrm{~Hz}, 1 \mathrm{H}), 3.89(\mathrm{dm}, J=6.2 \mathrm{~Hz}, 1 \mathrm{H}), 4.47(\mathrm{~d}, J=11.7 \mathrm{~Hz}, 1 \mathrm{H}), 4.62(\mathrm{~d}, J=11.7 \mathrm{~Hz}, 1$ 
H), $4.63(\mathrm{~m}, 1 \mathrm{H}), 5.12(\mathrm{dm}, J=10.6 \mathrm{~Hz}, 1 \mathrm{H}), 5.23(\mathrm{~d}, J=17.2 \mathrm{~Hz}, 1 \mathrm{H}), 5.90$ (ddd, $J=$ 6.2, 10.6, $17.2 \mathrm{~Hz}, 1 \mathrm{H}), 7.26-7.33(\mathrm{~m}, 5 \mathrm{H}) ;{ }^{13} \mathrm{C} \mathrm{NMR}\left(100 \mathrm{MHz}, \mathrm{CDCl}_{3}\right.$, one isomer) $\delta$ $12.4,15.1,18.1,40.0,71.0,72.5,72.9,78.2,114.5,127.7,127.8,128.4,138.9,141.2 ;{ }^{1} \mathrm{H}$ NMR (400 MHz, $\mathrm{CDCl}_{3}$, the other isomer) $\delta 1.05-1.12(\mathrm{~m}, 21 \mathrm{H}), 1.19(\mathrm{~d}, J=6.2 \mathrm{~Hz}, 3 \mathrm{H})$, 1.70-1.80 (m, $3 \mathrm{H}), 1.76(\mathrm{dm}, J=14.3 \mathrm{~Hz}, 1 \mathrm{H}), 3.29$ (br d, $J=2.0 \mathrm{~Hz}, 1 \mathrm{H}), 3.44$ (qm, $J=$ $6.2 \mathrm{~Hz}, 1 \mathrm{H}), 3.96(\mathrm{dm}, J=6.2 \mathrm{~Hz}, 1 \mathrm{H}), 4.54(\mathrm{~d}, J=11.7 \mathrm{~Hz}, 1 \mathrm{H}), 4.63(\mathrm{~m}, 1 \mathrm{H}), 4.64$ (d, $J=11.7 \mathrm{~Hz}, 1 \mathrm{H}), 5.14(\mathrm{dm}, J=10.6 \mathrm{~Hz}, 1 \mathrm{H}), 5.24(\mathrm{~d}, J=17.2 \mathrm{~Hz}, 1 \mathrm{H}), 5.90$ (ddd, $J=$ 6.2, 10.6, 17.2 Hz, $1 \mathrm{H}), 7.26-7.33(\mathrm{~m}, 5 \mathrm{H}) ;{ }^{13} \mathrm{C} \mathrm{NMR} \mathrm{(100} \mathrm{MHz}, \mathrm{CDCl}_{3}$, the other isomer) $\delta 12.4,14.9,18.1,39.4,71.0,72.5,73.0,78.0,114.3,127.7,127.8,128.4,138.8$, 140.7; HRMS, calcd for $\mathrm{C}_{23} \mathrm{H}_{40} \mathrm{O}_{3} \mathrm{Si}-i \operatorname{Pr}$ : 349.2199, found $\mathrm{m} / z$ (relative intensity) $349.2187\left(\mathrm{M}^{+}-i \operatorname{Pr}, 100\right), 332(6), 331$ (24).

3,5-anti-4-Methoxycarbonyl-2-(t-butyldimethylsiloxy)-6-octen-3-ol (2.40): $1: 1$ diastereomeric mixture regarding C2 stereocenter, IR (neat) 3560 (s), 2932 (s), 1736 (s), 966 (m) $\mathrm{cm}^{-1} ;{ }^{1} \mathrm{H} \mathrm{NMR}\left(400 \mathrm{MHz}, \mathrm{CDCl}_{3}\right.$, one isomer) $\delta 0.07(\mathrm{~s}, 6 \mathrm{H}), 0.89(\mathrm{~s}, 9 \mathrm{H}), 1.13(\mathrm{~d}, J=$ $6.1 \mathrm{~Hz}, 3 \mathrm{H}), 2.28(\mathrm{~d}, J=9.1 \mathrm{~Hz}, 1 \mathrm{H}), 2.35(\mathrm{dm}, J=9.1 \mathrm{~Hz}, 1 \mathrm{H}), 2.48-2.56(\mathrm{~m}, 2 \mathrm{H}), 3.48$ $(\mathrm{dt}, J=2.0,9.1 \mathrm{~Hz}, 1 \mathrm{H}), 3.67(\mathrm{~s}, 3 \mathrm{H}), 3.71(\mathrm{dm}, J=6.1 \mathrm{~Hz}, 1 \mathrm{H}), 5.37(\mathrm{dtm}, J=13.6,6.1$ $\mathrm{Hz}, 1 \mathrm{H}), 5.48(\mathrm{dq}, J=13.6,6.1 \mathrm{~Hz}, 1 \mathrm{H}) ;{ }^{13} \mathrm{C} \mathrm{NMR}\left(100 \mathrm{MHz}, \mathrm{CDCl}_{3}\right.$, one isomer) $\delta-$ $4.9,-4.4,17.5,18.0,21.0,25.8,31.6,47.8,50.9,69.2,75.2,127.6,127.8,174.7 ;{ }^{1} \mathrm{H}$ NMR (400 $\mathrm{MHz}, \mathrm{CDCl}_{3}$, the other isomer) $\delta 0.07(\mathrm{~s}, 6 \mathrm{H}), 0.90(\mathrm{~s}, 9 \mathrm{H}), 1.20(\mathrm{~d}, J=6.3 \mathrm{~Hz}, 3 \mathrm{H})$, $1.63(\mathrm{dm}, J=6.1 \mathrm{~Hz}, 3 \mathrm{H}), 2.28(\mathrm{~d}, J=9.1 \mathrm{~Hz}, 1 \mathrm{H}), 2.35(\mathrm{~d}, J=9.1 \mathrm{~Hz}, 1 \mathrm{H}), 2.48-2.56$ $(\mathrm{m}, 2 \mathrm{H}), 3.48(\mathrm{dt}, J=2.0,9.1 \mathrm{~Hz}, 1 \mathrm{H}), 3.66(\mathrm{~s}, 3 \mathrm{H}), 3.77(\mathrm{dq}, J=2.0,6.3 \mathrm{~Hz}, 1 \mathrm{H}), 5.37$ $(\mathrm{dtm}, J=13.6,6.1 \mathrm{~Hz}, 1 \mathrm{H}), 5.48(\mathrm{dq}, J=13.6,6.1 \mathrm{~Hz}, 1 \mathrm{H}) ;{ }^{13} \mathrm{C} \mathrm{NMR}\left(100 \mathrm{MHz}, \mathrm{CDCl}_{3}\right.$, the other isomer) $\delta-5.0,-4.1,17.9,18.0,21.0,25.8,32.1,49.9,51.4,69.2,75.2,127.3$, 127.5, 174.7; HRMS, calcd for $\mathrm{C}_{16} \mathrm{H}_{32} \mathrm{O}_{4} \mathrm{Si}$ : 316.2070 , found $\mathrm{m} / z$ (relative intensity) $316.2057\left(\mathrm{M}^{+}, 100\right), 301$ (15), 287 (75), 285 (73). Anal. calcd for $\mathrm{C}_{16} \mathrm{H}_{32} \mathrm{O}_{4} \mathrm{Si}$ : C, 60.72; 
H, 10.19, found: C, 61.05; H, 9.88 .

2,4-Dimethyl-5-hexen-2-ol (2.41): IR (neat) 3350 (s), 3070 (w), 2970 (s), 2930 (s), 1650 (w), $1370(\mathrm{~m}), 1150(\mathrm{~m}), 900(\mathrm{~m}) \mathrm{cm}^{-1} ;{ }^{1} \mathrm{H}$ NMR (400 MHz, $\left.\mathrm{CDCl}_{3}\right) \delta 1.03(\mathrm{~d}, J=6.6 \mathrm{~Hz}$ $3 \mathrm{H}), 1.20(\mathrm{~s}, 3 \mathrm{H}), 1.25$ (s, $3 \mathrm{H}), 1.49(\mathrm{dd}, J=4.0,13.9 \mathrm{~Hz}, 1 \mathrm{H}), 1.63(\mathrm{dd}, J=7.7,13.9 \mathrm{~Hz}$, $1 \mathrm{H}), 1.79(\mathrm{~s}, 1 \mathrm{H}), 2.44(\mathrm{~m}, 1 \mathrm{H}), 4.96(\mathrm{dd}, J=1.8,10.3 \mathrm{~Hz}, 1 \mathrm{H}), 5.07(\mathrm{dd}, J=1.8,17.2$ $\mathrm{Hz}, 1 \mathrm{H}), 5.81$ (ddd, $J=8.8,10.3,17.2 \mathrm{~Hz}, 1 \mathrm{H}$ ); HRMS, calcd for $\mathrm{C}_{8} \mathrm{H}_{16} \mathrm{O}: 128.1201$, found $m / z$ (relative intensity) $128.1160\left(\mathrm{M}^{+}, 100\right)$.

1-(2-Methyl-3-butenyl)cyclohexanol (2.42): IR (neat) 3440 (m), 2920 (s), 1450 (s), 1420 (m), 1370 (m), 1260 (m), 1170 (m), 970 (s), 900 (s), 830 (m) cm ${ }^{-1}$; ${ }^{1} \mathrm{H}$ NMR (400 MHz, $\left.\mathrm{CDCl}_{3}\right) \delta 1.02(\mathrm{~d}, J=6.9 \mathrm{~Hz}, 3 \mathrm{H}), 1.40-1.60(\mathrm{~m}, 12 \mathrm{H}), 2.47(\mathrm{~m}, 1 \mathrm{H}), 4.94(\mathrm{dd}, J=1.4$, $10.3 \mathrm{~Hz}, 1 \mathrm{H}), 5.05$ (dd, $J=1.4,17.2 \mathrm{~Hz}, 1 \mathrm{H}), 5.82$ (ddd, $J=8.8,10.3,17.2 \mathrm{~Hz}, 1 \mathrm{H})$; HRMS, calcd for $\mathrm{C}_{11} \mathrm{H}_{20} \mathrm{O}: 168.1514$, found $\mathrm{m} / \mathrm{z}$ (relative intensity) $168.1506\left(\mathrm{M}^{+}, 24\right)$, $151(9), 135$ (28), 125 (100), $121(21)$.

cis-1-(2,3-Dimethyl-3-butenyl)-4-methylcyclohexanol (2.43eq): IR (neat) 3474 (s), 2924 (s), 1643 (m), 1443 (s), 1373 (s), 989 (s), 887 (s) cm-1; ${ }^{1} \mathrm{H}$ NMR (400 MHz, CDCl 3 ) $\delta 0.92$ (br d, $J=5.6 \mathrm{~Hz}, 3 \mathrm{H}), 1.03(\mathrm{~d}, J=6.7 \mathrm{~Hz}, 3 \mathrm{H}), 1.24-1.49(\mathrm{~m}, 8 \mathrm{H}), 1.63-1.77(\mathrm{~m}, 4 \mathrm{H})$, 1.73 (br s, $3 \mathrm{H}), 2.60(\mathrm{~m}, 1 \mathrm{H}), 4.72$ (br s, $1 \mathrm{H}), 4.83$ (br s, $1 \mathrm{H}) ;{ }^{13} \mathrm{C}$ NMR $(75 \mathrm{MHz}$ $\left.\mathrm{CDCl}_{3}\right) \delta 17.9,21.9,22.4,30.1,30.2,32.3,36.5,36.8,38.1,48.1,71.3,110.5,152.4$; HRMS calcd for $\mathrm{C}_{13} \mathrm{H}_{24} \mathrm{O}-\mathrm{OH}: 179.1436$. found $m / z$ (relative intensity) $179.1351\left(\mathrm{M}^{+}{ }_{-}\right.$ $\mathrm{OH}, 15), 178$ (100).

trans-1-(2,3-Dimethyl-3-butebyl)-4-methyl-cyclohexanol (2.43ax): IR (neat) 3445 (s), 2926 (s), 1643 (m), 1456 (s), 1375 (m), 1099 (s), 1003 (s), 887 (s), cm ${ }^{-1}$; ${ }^{1} \mathrm{H}$ NMR (400 $\left.\mathrm{MHz}, \mathrm{CDCl}_{3}\right) \delta 0.91-1.11(\mathrm{~m}, 2 \mathrm{H}), 0.91(\mathrm{~d}, J=6.6 \mathrm{~Hz}, 3 \mathrm{H}), 1.07(\mathrm{~d}, J=6.4 \mathrm{~Hz}, 3 \mathrm{H})$, 1.36-1.59 (m, $3 \mathrm{H}), 1.61-1.72$ (m, $5 \mathrm{H}), 1.76$ (br s, $3 \mathrm{H}), 1.85$ (m, $1 \mathrm{H}), 2.05$ (br s, $1 \mathrm{H})$, $2.53(\mathrm{~m}, 1 \mathrm{H}), 4.75(\mathrm{~d}, J=1.7 \mathrm{~Hz}, 1 \mathrm{H}), 4.87(\mathrm{~d}, J=1.7 \mathrm{~Hz}, 1 \mathrm{H}) ;{ }^{13} \mathrm{C}$ NMR $(75 \mathrm{MHz}$ 
$\left.\mathrm{CDCl}_{3}\right) \delta 18.0,21.3,21.8,31.4,31.5,31.6,36.7,36.8,38.2,41.8,72.7,110.6,152.5$.

cis-1-(2,3-Dimethyl-3-butenyl)-4-phenylcyclohexanol (2.44eq): IR (neat) 3470 (s), 2860 (s), 1643 (m), 1600 (m), 1493 (s), 1445 (s), 1375 (s), 1219 (s), 976 (s), 889 (s), 758 (s), 700

(s) $\mathrm{cm}^{-1} ;{ }^{1} \mathrm{H}$ NMR $\left(400 \mathrm{MHz}, \mathrm{CDCl}_{3}\right) \delta 1.05$ (d, J=7.0 Hz, $\left.3 \mathrm{H}\right), 1.35-1.91(\mathrm{~m}, 11 \mathrm{H})$, 1.75 (br s, $3 \mathrm{H}), 2.44$ (tt, $J=3.3,12.1 \mathrm{~Hz}, 1 \mathrm{H}), 2.63$ (m, $1 \mathrm{H}), 4.75$ (br s, $1 \mathrm{H}), 4.87$ (br s, 1 H), 7.14-7.30 (m, $5 \mathrm{H}) ;{ }^{13} \mathrm{C} \mathrm{NMR}\left(100 \mathrm{MHz}, \mathrm{CDCl}_{3}\right) \delta 18.1,22.0,29.2,36.9,37.1$, 38.6, 44.3, 48.4, 71.1, 110.6, 125.9, 126.9, 128.3, 147.4, 152.3; HRMS calcd for $\mathrm{C}_{18} \mathrm{H}_{26} \mathrm{O}$ : 258.1984, found $\mathrm{m} / z$ (relative intensity) 258.1992 (M+14), 240 (100), 225 (18).

trans-1-(2,3-Dimethyl-3-butenyl)-4-phenylcyclohexanol (2.44ax): IR (neat) 3458 (s), 2932 (s), 1495 (m), 1452 (s), 1020 (m), 889 (m), 758 (m), 700 (s) cm-1; ${ }^{1} \mathrm{H}$ NMR (400 $\left.\mathrm{MHz} \mathrm{CDCl}_{3}\right) \delta 1.09(\mathrm{~d}, J=7.0 \mathrm{~Hz}, 3 \mathrm{H}), 1.52-2.26(\mathrm{~m}, 7 \mathrm{H}), 1.77$ (br s, $\left.3 \mathrm{H}\right), 2.47-2.60$ (m, $4 \mathrm{H}), 3.03$ (dt, $J=\mathrm{dt}, J=12.1,3.5 \mathrm{~Hz}, 1 \mathrm{H}), 4.77$ (br s, $1 \mathrm{H}), 4.88$ (br s, $1 \mathrm{H}), 7.17-7.35$ $(\mathrm{m}, 5 \mathrm{H})$.

cis-4-t-Butyl-1-(2,3-dimethyl-3-butenyl)cyclohexanol (2.45eq): IR (neat) 3471 (s), 2941 (s), 1643 (m), 1443 (s), 1366 (s), 1232 (m), 955 (s), 887 (s) cm-1; ${ }^{1} \mathrm{H}$ NMR (400 MHz, $\left.\mathrm{CDCl}_{3}\right) \delta 0.85(\mathrm{~s}, 9 \mathrm{H}), 0.87-0.95(\mathrm{~m}, 2 \mathrm{H}), 1.02(\mathrm{~d}, J=7.0 \mathrm{~Hz}, 3 \mathrm{H}), 1.21-1.39(\mathrm{~m}, 4 \mathrm{H})$, 1.52-1.82 (m, $6 \mathrm{H}), 1.72$ (br s, $3 \mathrm{H}), 2.59$ (m, $1 \mathrm{H}), 4.71$ (br s, $1 \mathrm{H}), 4.82$ (br s, $1 \mathrm{H}) ;{ }^{13} \mathrm{C}$ NMR (100 MHz, $\left.\mathrm{CDCl}_{3}\right) \delta 18.1,22.0,22.4,22.7,27.6,32.4,36.9,37.4,38.7,48.1,48.5$, 71.3, 110.3, 152.5; HRMS calcd for $\mathrm{C}_{16} \mathrm{H}_{30} \mathrm{O}$ : 238.2297, found $\mathrm{m} / z$ (relative intensity) $238.2309\left(\mathrm{M}^{+}, 8\right), 220$ (100), 205 (43), 148 (25).

trans-4-t-Butyl-1-(2,3-dimethyl-3-butenyl)cyclohexanol (2.45ax): IR (neat) 3437 (s), 2941 (s), 1643 (m), 1456 (s), 1366 (s), 1078 (m), 1030 (m), 988 (m), 887 (s) cm-1; ${ }^{1} \mathrm{H}$ NMR (400 MHz, $\left.\mathrm{CDCl}_{3}\right) \delta 0.85(\mathrm{~s}, 9 \mathrm{H}), 1.01(\mathrm{~m}, 2 \mathrm{H}), 1.06(\mathrm{~d}, J=7.0 \mathrm{~Hz}, 3 \mathrm{H})$, 1.31-1.39 (m, 2 H), 1.55-1.76 (m, $6 \mathrm{H}), 1.74$ (br s, $3 \mathrm{H}), 1.94$ (dd, J = 2.9, $12.8 \mathrm{~Hz}, 1 \mathrm{H})$, $2.01(\mathrm{~s}, 1 \mathrm{H}), 2.50(\mathrm{~m}, 1 \mathrm{H}), 4.74(\mathrm{~s}, 1 \mathrm{H}), 4.84(\mathrm{~s}, 1 \mathrm{H}) ;{ }^{13} \mathrm{C} \mathrm{NMR}\left(100 \mathrm{MHz}, \mathrm{CDCl}_{3}\right) \delta$ 
$18.3,21.9,24.4,24.5,27.7,32.3,36.8,38.3,39.5,40.9,47.6,72.9,110.4,152.5$.

5-(But-3-enyl)-2,2-dimethyl-1,3-dioxan-5-ol (2.46): a mixture with 5-(but-2-enyl)-2,2dimethyl-1,3-dioxan-5-ol (3.2) in a ratio of 1.3:1; IR (neat) 3456 (s), 2939 (s), 1373 (s), $1088(\mathrm{~s}), 833(\mathrm{~m}) \mathrm{cm}^{-1} ;{ }^{1} \mathrm{H}$ NMR (400 MHz, $\left.\mathrm{CDCl}_{3}\right) \delta 1.44-1.49$ (m, $\left.2 \mathrm{H}\right), 1.46(\mathrm{~s}, 3 \mathrm{H})$, 1.47 (s, $3 \mathrm{H}), 2.17(\mathrm{dm}, J=6.6 \mathrm{~Hz}, 2 \mathrm{H}), 3.20(\mathrm{~s}, 1 \mathrm{H}), 3.57(\mathrm{dm}, J=10.7 \mathrm{~Hz}, 2 \mathrm{H}), 3.80$ (br d, $J=10.7 \mathrm{~Hz}, 2 \mathrm{H}), 4.99(\mathrm{dm}, J=10.2 \mathrm{~Hz}, 1 \mathrm{H}), 5.07$ (d, $J=17.0 \mathrm{~Hz}, 1 \mathrm{H}), 5.82$ (ddt, $J=10.2,17.0,6.6 \mathrm{~Hz}, 1 \mathrm{H}) ;{ }^{13} \mathrm{C} \mathrm{NMR}\left(100 \mathrm{MHz}, \mathrm{CDCl}_{3}\right) \delta 17.0,17.9,25.0,31.8,65.4$, 67.1, 67.3, 96.9, 113.5, 137.0; HRMS, calcd for $\mathrm{C}_{10} \mathrm{H}_{18} \mathrm{O}_{3}: 186.1256$, found $\mathrm{m} / z$ (relative intensity) $186.1234\left(\mathrm{M}^{+}, 1\right), 171(61), 155$ (100).

5-(But-2-enyl)-2,2-dimethyl-1,3-dioxan-5-ol (3.2): ${ }^{1} \mathrm{H}$ NMR (400 $\left.\mathrm{MHz}, \mathrm{CDCl}_{3}\right) \delta 1.46$ (br s, $3 \mathrm{H}), 1.47$ (br s, $3 \mathrm{H}), 1.71$ (dm, $J=6.6 \mathrm{~Hz}, 3 \mathrm{H}), 2.12-2.20$ (m, $2 \mathrm{H}), 3.06$ (br s, 1 H), 3.59 (br d, $J=11.7 \mathrm{~Hz}, 2 \mathrm{H}), 3.84$ (br d, $J=11.7 \mathrm{~Hz}, 2 \mathrm{H}), 5.49$ (dqm, $J=15.1,6.6 \mathrm{~Hz}$, $1 \mathrm{H}), 5.57(\mathrm{dm}, J=15.1 \mathrm{~Hz}, 1 \mathrm{H}) ;{ }^{13} \mathrm{C} \mathrm{NMR}\left(100 \mathrm{MHz}, \mathrm{CDCl}_{3}\right) \delta 16.7,26.4,27.2,36.3$, $67.1,67.3,97.0,122.5,128.3$.

2,2-Dimethyl-5-(2-methylbut-3-enyl)-1,3-dioxan-5-ol (2.47): a mixture with 2,2-dimethyl-5-(3-methylbut-3-enyl)-1,3-dioxan-5-ol (4.9) in a ratio of 4.7:1; IR (neat) 3472 (s), 2962 (s), 1103 (s), 833 (m) cm-1; ${ }^{1} \mathrm{H}$ NMR (400 MHz, CDCl 3 ) $\delta 1.04(\mathrm{~d}, J=6.8 \mathrm{~Hz}, 3 \mathrm{H})$, $1.35-1.54(\mathrm{~m}, 2 \mathrm{H}), 1.42(\mathrm{~s}, 3 \mathrm{H}), 1.43(\mathrm{~s}, 3 \mathrm{H}), 2.47$ (dqm, J = 8.2, $6.8 \mathrm{~Hz}, 1 \mathrm{H}), 3.15$ (s, 1 H), $3.50(\mathrm{dm}, J=11.8 \mathrm{~Hz}, 1 \mathrm{H}), 3.67(\mathrm{dm}, J=11.8 \mathrm{~Hz}, 1 \mathrm{H}), 3.75(\mathrm{br} \mathrm{d}, J=11.8 \mathrm{~Hz}, 1 \mathrm{H})$, 3.82 (br d, $J=11.8 \mathrm{~Hz}, 1 \mathrm{H}), 4.95$ (br d, $J=10.1 \mathrm{~Hz}, 1 \mathrm{H}), 5.06(\mathrm{~d}, J=17.0 \mathrm{~Hz}, 1 \mathrm{H}), 5.75$ $(\mathrm{ddd}, J=8.2,10.1,17.0 \mathrm{~Hz}, 1 \mathrm{H}) ;{ }^{13} \mathrm{C} \mathrm{NMR}\left(100 \mathrm{MHz}, \mathrm{CDCl}_{3}\right) \delta$ 18.5, 22.5, 28.0, 32.7, 40.7, 68.5, 68.7, 98.0, 112.8, 145.1; HRMS, calcd for $\mathrm{C}_{11} \mathrm{H}_{20} \mathrm{O}_{3}: 200.1412$, found $\mathrm{m} / z$ (relative intensity) $200.1391\left(\mathrm{M}^{+}, 3\right), 185$ (67), 169 (100).

2,2-Dimethyl-5-(3-methylbut-3-enyl)-1,3-dioxan-5-ol (4.9): ${ }^{1} \mathrm{H} \mathrm{NMR}\left(400 \mathrm{MHz}, \mathrm{CDCl}_{3}\right)$ $\delta 1.40-1.54(\mathrm{~m}, 2 \mathrm{H}), 1.44(\mathrm{~s}, 3 \mathrm{H}), 1.45(\mathrm{~s}, 3 \mathrm{H}), 1.73($ br s, $3 \mathrm{H}), 2.05-2.17(\mathrm{~m}, 2 \mathrm{H})$, 
3.17 (br s, $1 \mathrm{H}$ ), 3.57 (br d, $J=11.7 \mathrm{~Hz}, 1 \mathrm{H}$ ), 3.82 (br d, $J=11.7 \mathrm{~Hz}, 1 \mathrm{H}), 4.69$ (br s, $1 \mathrm{H}$ ), 4.72 (br s, $1 \mathrm{H}) ;{ }^{13} \mathrm{C}$ NMR $\left(100 \mathrm{MHz}, \mathrm{CDCl}_{3}\right) \delta 18.1,22.3,28.4,29.7,31.8,67.1,68.5$, 98.1, 109.7, 145.3.

2,2-Dimethyl-5-(6-methyl-2-vinylhept-5-enyl)-1,3-dioxan-5-ol (2.48): IR (neat) 3472 (s), 2916 (s), 1373 (s), 1103 (s), 833 (m) cm ${ }^{-1} ;{ }^{1} \mathrm{H}$ NMR (400 MHz, CDCl 3 ) $\delta$ 1.22-1.42 (m, 3 H), 1.44 (s, $3 \mathrm{H}), 1.46$ (s, $3 \mathrm{H}), 1.56$ (m, $1 \mathrm{H}), 1.61$ (br s, $3 \mathrm{H}), 1.70$ (br s, $3 \mathrm{H}), 1.85$ - 2.10 $(\mathrm{dm}, J=7.4 \mathrm{~Hz}, 2 \mathrm{H}), 2.33(\mathrm{dm}, J=9.1 \mathrm{~Hz}, 1 \mathrm{H}), 3.16(\mathrm{~s}, 1 \mathrm{H}), 3.51(\mathrm{dd}, J=2.2,11.8 \mathrm{~Hz}$, $1 \mathrm{H}), 3.71(\mathrm{dd}, J=2.2,11.8 \mathrm{~Hz}, 1 \mathrm{H}), 3.77(\mathrm{~d}, J=11.8 \mathrm{~Hz}, 1 \mathrm{H}), 3.86(\mathrm{~d}, J=11.8 \mathrm{~Hz}, 1 \mathrm{H})$, $5.05($ br d, $J=10.8 \mathrm{~Hz}, 1 \mathrm{H}), 5.06(\mathrm{~d}, J=16.5 \mathrm{~Hz}, 1 \mathrm{H}), 5.10(\mathrm{tm}, J=7.4 \mathrm{~Hz}, 1 \mathrm{H}), 5.62$ $(\mathrm{ddd}, J=9.1,10.8,16.5 \mathrm{~Hz}, 1 \mathrm{H}) ;{ }^{13} \mathrm{C} \mathrm{NMR}\left(100 \mathrm{MHz}, \mathrm{CDCl}_{3}\right) \delta 16.7,18.0,24.4,24.7$, 27.0, 35.7, 37.4, 38.7, 66.5, 67.8, 67.9, 97.2, 114.1, 123.2. 130.6, 142.8; HRMS, calcd for $\mathrm{C}_{16} \mathrm{H}_{28} \mathrm{O}_{3}: 268.2038$, found $\mathrm{m} / \mathrm{z}$ (relative intensity) $268.2035\left(\mathrm{M}^{+}, 48\right), 253$ (100), 237 (26), $210(55)$.

2,2-Dimethyl-5-(2,3-dimethylbut-3-enyl)-1,3-dioxan-5-ol (2.49): IR (neat) 3472 (s), 2963 (s), 1373 (s), 1103 (s), $833(\mathrm{~m}) \mathrm{cm}^{-1} ;{ }^{1} \mathrm{H}$ NMR (400 MHz, $\left.\mathrm{CDCl}_{3}\right) \delta 1.08$ (d, J=6.9 Hz, 3 H), $1.42(\mathrm{dm}, J=14.5 \mathrm{~Hz}, 1 \mathrm{H}), 1.44$ (s, $3 \mathrm{H}), 1.45$ (s, $3 \mathrm{H}), 1.58(\mathrm{dm}, J=14.5 \mathrm{~Hz}, 1 \mathrm{H})$, 1.73 (br s, $3 \mathrm{H}), 2.55$ (qm, $J=6.9 \mathrm{~Hz}, 1 \mathrm{H}), 3.18(\mathrm{~s}, 3 \mathrm{H}), 3.53(\mathrm{dm}, J=11.8 \mathrm{~Hz}, 1 \mathrm{H}), 3.67$ $(\mathrm{dm}, J=11.8 \mathrm{~Hz}, 1 \mathrm{H}), 3.80(\mathrm{~d}, J=11.8 \mathrm{~Hz}, 1 \mathrm{H}), 3.85(\mathrm{~d}, J=11.8 \mathrm{~Hz}, 1 \mathrm{H}), 4.73(\mathrm{~s}, 1 \mathrm{H})$, $4.81(\mathrm{~s}, 1 \mathrm{H}) ;{ }^{13} \mathrm{C} \mathrm{NMR}\left(100 \mathrm{MHz}, \mathrm{CDCl}_{3}\right) \delta 17.3,17.6,20.7,26.7,34.5,37.7,66.0,67.0$, 67.5, 96.8, 108.8, 149.6; HRMS, calcd for $\mathrm{C}_{12} \mathrm{H}_{22} \mathrm{O}_{3}$ : 214.1569, found $\mathrm{m} / \mathrm{z}$ (relative intensity) $214.1539\left(\mathrm{M}^{+}, 48\right), 199(100), 183(90)$.

1-(2-Cyclohexenyl)-1-phenylmethanol (2.50): a mixture of syn and anti = 4:1; IR (neat) 3391 (s), 3025 (s), 2928 (s), 1454 (s), 1016 (s), 710 (m) cm-1; ${ }^{1} \mathrm{H}$ NMR (400 MHz, CDCl 3 , syn isomer) $\delta 1.45-1.59(\mathrm{~m}, 2 \mathrm{H}), 1.65-1.79(\mathrm{~m}, 2 \mathrm{H}), 1.82(\mathrm{~d}, J=2.5 \mathrm{~Hz}, 1 \mathrm{H}), 1.98-2.01$ 
(m, $2 \mathrm{H}), 2.50(\mathrm{ddm}, J=2.2,6.6 \mathrm{~Hz}, 1 \mathrm{H}), 4.59(\mathrm{dd}, J=2.5,6.6 \mathrm{~Hz}, 1 \mathrm{H}), 5.38(\mathrm{dd}, J=2.2$, $10.0 \mathrm{~Hz}, 1 \mathrm{H}), 5.81$ (ddd, $J=3.7,6.4,10.0 \mathrm{~Hz}, 1 \mathrm{H}), 7.25-7.37(\mathrm{~m}, 5 \mathrm{H}) ;{ }^{13} \mathrm{C} \mathrm{NMR}(100$ $\mathrm{MHz}, \mathrm{CDCl}_{3}$, syn isomer) $\delta 21.1,23.9,25.3,43.0,77.3,126.4,127.2,127.9,128.0,130.2$, $142.7 ;{ }^{1} \mathrm{H} \mathrm{NMR}\left(400 \mathrm{MHz}, \mathrm{CDCl}_{3}\right.$, anti isomer) $\delta 1.20-1.39(\mathrm{~m}, 1 \mathrm{H}), 1.43-1.79(\mathrm{~m}, 3 \mathrm{H})$, 1.84 (br d, $J=3.4 \mathrm{~Hz}, 1 \mathrm{H}), 1.98-2.01(\mathrm{~m}, 2 \mathrm{H}), 2.50(\mathrm{dm}, J=6.6 \mathrm{~Hz}, 1 \mathrm{H}), 4.47$ (dd, $J=$ 3.4, 6.6 Hz, $1 \mathrm{H}), 5.81-5.87$ (m, $2 \mathrm{H}), 7.25-7.37$ (m, $5 \mathrm{H}) ;{ }^{13} \mathrm{C} \mathrm{NMR}\left(100 \mathrm{MHz}, \mathrm{CDCl}_{3}\right.$, anti isomer) $\delta 21.5,23.9,26.3,42.8,77.9,126.1,126.9,127.9,128.0,129.7,143.4$; HRMS, calcd for $\mathrm{C}_{13} \mathrm{H}_{16} \mathrm{O}$ : 188.1201, found $\mathrm{m} / \mathrm{z}$ (relative intensity) $188.1075\left(\mathrm{M}^{+}, 100\right), 187$ (10), $171(8)$.

(6E)-3,5-anti-7-Methoxy-1-phenyl-5-trimethylsiloxy-6-hepten-3-ol (2.51): a mixture of anti and syn = 5:1; IR (neat) 3379 (s), 2932 (s), 1207 (s), 1030 (s), 934 (s), $698(\mathrm{~s}) \mathrm{cm}^{-1} ;{ }^{1} \mathrm{H}$ NMR (400 MHz, $\mathrm{CDCl}_{3}$, anti isomer) $\delta 0.13(\mathrm{~s}, 9 \mathrm{H}), 1.83-1.55(\mathrm{~m}, 4 \mathrm{H}), 2.66(\mathrm{ddm}, J=$ 10.0, 13.7 Hz, $1 \mathrm{H}), 2.80$ (ddm, $J=10.0,13.7 \mathrm{~Hz}, 1 \mathrm{H}), 3.23$ (d, J = 3.2 Hz, $1 \mathrm{H}), 3.52$ (s, 3 H), $3.94(\mathrm{dm}, J=3.2 \mathrm{~Hz}, 1 \mathrm{H}), 4.46(\mathrm{dm}, J=8.1 \mathrm{~Hz}, 1 \mathrm{H}), 4.85(\mathrm{dd}, J=8.1,12.7 \mathrm{~Hz}, 1 \mathrm{H})$, $6.45(\mathrm{~d}, J=12.7 \mathrm{~Hz}, 1 \mathrm{H}), 7.15-7.29(\mathrm{~m}, 5 \mathrm{H}) ;{ }^{13} \mathrm{C} \mathrm{NMR}\left(100 \mathrm{MHz}, \mathrm{CDCl}_{3}\right.$, anti isomer) $\delta$ $0.4,32.0,39.3,44.9,55.9,68.1,69.8,105.4,125.6,128.2,128.3,142.2,148.6 ;{ }^{13} \mathrm{C} \mathrm{NMR}$ (100 $\mathrm{MHz}, \mathrm{CDCl}_{3}$, syn isomer) $\delta 9.8,30.3,38.6,44.9,55.9,68.1,72.6,105.4,125.6,128.2$, 128.3, 142.2, 148.6; HRMS, calcd for $\mathrm{C}_{17} \mathrm{H}_{28} \mathrm{O}_{3} \mathrm{Si}$ : 308.1808 , found $m / z$ (relative intensity) $308.1811\left(\mathrm{M}^{+}, 57\right), 309(28), 291$ (28), 290 (100), 276 (71).

1-Phenyl-3-pentenol (3.1): IR (neat) 3400 (s), 2945 (m), 1650 (w), 1455 (s), 1060 (s), $1030(\mathrm{~m}), 962(\mathrm{~m}), 750(\mathrm{~m}), 698(\mathrm{~s}) \mathrm{cm}^{-1} ;{ }^{1} \mathrm{H}$ NMR $\left(400 \mathrm{MHz}, \mathrm{CDCl}_{3}\right) \delta 1.69$ (br d, $J=$ $6.6 \mathrm{~Hz}, 3 \mathrm{H}), 2.05(\mathrm{~d}, J=2.9 \mathrm{~Hz}, 1 \mathrm{H}), 2.39(\mathrm{~m}, 1 \mathrm{H}$, coalescing to $\mathrm{dd}, J=7.7,14.1 \mathrm{~Hz}$ by irradiation at 4.67), 2.47 (dddm, $J=4.4,6.2,14.1 \mathrm{~Hz}, 1 \mathrm{H}$, coalescing to br dd, $J=6.2,14.1$ $\mathrm{Hz}$ by irradiation at 4.67), $4.67(\mathrm{ddd}, J=2.9,4.4,7.7 \mathrm{~Hz}, 1 \mathrm{H}), 5.43(\mathrm{~m}, 1 \mathrm{H}$, coalescing to ddd, $J=6.2,7.7,15.2 \mathrm{~Hz}$ by irradiation at 1.69$), 5.61(\mathrm{~m}, 1 \mathrm{H}$, coalescing to br $\mathrm{d}, J=15.2$ 
$\mathrm{Hz}$ by irradiation at 1.69), 7.22-7.40 (m, $5 \mathrm{H})$. Anal. calcd for $\mathrm{C}_{11} \mathrm{H}_{14} \mathrm{O}: \mathrm{C}, 81.44 ; \mathrm{H}$, 8.70, found: $\mathrm{C}, 81.05 ; \mathrm{H}, 8.82$.

1,4-Diphenyl-4-penten-1-ol (4.1): IR (neat) 3371 (s), 1057 (s), 903 (m), 702 (s) cm-1; ${ }^{1} \mathrm{H}$ NMR (400 MHz, $\left.\mathrm{CDCl}_{3}\right) \delta 1.83(\mathrm{~d}, J=3.3 \mathrm{~Hz}, 1 \mathrm{H}), 1.87(\mathrm{ddt}, J=9.8,15.3,5.3 \mathrm{~Hz}, 1 \mathrm{H})$, 1.95 (dddd, $J=5.3,8.1,9.8,15.3 \mathrm{~Hz}, 1 \mathrm{H}), 2.54$ (ddd, $J=5.3,9.8,15.2 \mathrm{~Hz}, 1 \mathrm{H}), 2.66$ (ddd, $J=5.3,9.8,15.2 \mathrm{~Hz}, 1 \mathrm{H}), 4.72(\mathrm{ddd}, J=3.3,5.3,8.1 \mathrm{~Hz}, 1 \mathrm{H}), 5.08$ (br s, $1 \mathrm{H}), 5.28$ (br s, $1 \mathrm{H}), 7.24-7.38(\mathrm{~m}, 10 \mathrm{H}) ;{ }^{13} \mathrm{C} \mathrm{NMR}\left(100 \mathrm{MHz} \mathrm{CDCl}_{3}\right) \delta 31.6,37.5,74.0,112.4,126.0$, 127.2, 127.4, 128.2, 128.3, 140.9, 144.4, 147.9; HRMS, calcd for $\mathrm{C}_{17} \mathrm{H}_{18} \mathrm{O}: 238.1358$, found $\mathrm{m} / \mathrm{z}$ (relative intensity) $238.1333\left(\mathrm{M}^{+}, 100\right), 239$ (19), 221 (3), 220 (13).

2-Methyl-1-phenyl-4-pentenol (4.2): a mixture of 1,2-anti/syn = 5.2:1; IR (neat) 3390 (s), 2990 (s), 1650 (m), 1450 (s), 1370 (m), 1010 (s), 990 (s), 910 (s), 750 (s), 690 (s) cm-1; ${ }^{1} \mathrm{H}$ NMR (400 MHz, $\mathrm{CDCl}_{3}, 1,2$-anti-isomer): $\delta 0.75(\mathrm{~d}, J=7.0 \mathrm{~Hz}, 3 \mathrm{H}), 1.91(\mathrm{~d}, J=2.9 \mathrm{~Hz}$, $1 \mathrm{H}), 1.94$ (br ddd, $J=4.0,6.6,13.4 \mathrm{~Hz}, 1 \mathrm{H}), 1.99$ (br dt, $J=13.4,7.9 \mathrm{~Hz}, 1 \mathrm{H}), 2.41$ (m, 1 $\mathrm{H}$, coalescing to br ddd, $J=4.0,7.1,7.9 \mathrm{~Hz}$ by irradiation at 0.75$), 4.44(\mathrm{dd}, J=2.9,7.1 \mathrm{~Hz}$, $1 \mathrm{H}), 5.04(\mathrm{br} \mathrm{d}, J=10.3 \mathrm{~Hz}, 1 \mathrm{H}), 5.07$ (br d, $J=16.7 \mathrm{~Hz}, 1 \mathrm{H}), 5.84$ (dddd, $J=6.6,7.9$, 10.3, 16.7 Hz, $1 \mathrm{H}), 7.25-7.38(\mathrm{~m}, 5 \mathrm{H}) ;{ }^{13} \mathrm{C} \mathrm{NMR}\left(100 \mathrm{MHz}, \mathrm{CDCl}_{3}\right) \delta 15.7,37.0,40.0$, 78.7, 116.2, 126.7, 127.5, 128.3, 137.2, 143.4; HRMS calcd for $\mathrm{C}_{12} \mathrm{H}_{16} \mathrm{O}$ : 176.1201, found $m / z$ (relative intensity) $176.1203\left(\mathrm{M}^{+}, 14\right), 134(17), 107$ (100), 79 (27). Anal calcd for $\mathrm{C}_{12} \mathrm{H}_{16} \mathrm{O}: \mathrm{C}, 81.77 ; \mathrm{H}, 9.15$, found $\mathrm{C}, 81.38 ; \mathrm{H}, 9.23$.

1,2-anti-1,2-Diphenyl-4-pentenol (4.3): IR (neat) 3440 (s), 3060 (m), 3030 (m), 2910 (m), 1950 (w), 1870 (w), 1810 (w), 1640 (m), 1600 (w), 1490 (m), 1450 (m), 1030 (m), 910 (m), 750 (s), 690 (s) cm ${ }^{-1} ;{ }^{1} \mathrm{H}$ NMR (400 MHz, $\left.\mathrm{CDCl}_{3}\right) \delta 1.98$ (d, J=3.3 Hz, $1 \mathrm{H}$ ), 2.56 (dddd, $J=1.1,7.0,10.6,14.7 \mathrm{~Hz}, 1 \mathrm{H}), 2.68$ (dddd, $J=1.1,4.8,7.0,14.7 \mathrm{~Hz}, 1 \mathrm{H}), 3.05$ (ddd, $J=$ 4.8, 7.0, 14.7 Hz, $1 \mathrm{H}), 4.84(\mathrm{dd}, J=3.3,6.6 \mathrm{~Hz}, 1 \mathrm{H}), 4.88(\mathrm{ddt}, J=2.0,10.2,1.1 \mathrm{~Hz}, 1 \mathrm{H})$, 4.97 (ddt, $J=2.0,17.1,1.1 \mathrm{~Hz}, 1 \mathrm{H}), 5.62$ (ddt, $J=10.2,17.1,7.0 \mathrm{~Hz}, 1 \mathrm{H}), 7.12-7.38$ (m, 
$10 \mathrm{H}$ ); HRMS calcd for $\mathrm{C}_{17} \mathrm{H}_{18} \mathrm{O}-\mathrm{H}_{2} \mathrm{O}: 220.1252$, found $\mathrm{m} / z$ (relative intensity) 220.1282 $\left(\mathrm{M}^{+}-\mathrm{H}_{2} \mathrm{O}, 32\right), 132(76), 107$ (100), 91 (47), 77 (30).

(4E)-1,2-anti-6-Methoxy-2-methyl-1-phenyl-4-hexen-1-ol (4.4): IR (neat) 3400 (s), 3070 (m), 3050 (m), 2980 (s), 2950 (s), 1720 (s), 1690 (s), 1600 (w), 1450 (s), 1380 (m), 1100 (s), 1020 (s), 980 (s), 760 (s), 700 (s) cm-1; ${ }^{1} \mathrm{H}$ NMR (400 MHz, $\left.\mathrm{CDCl}_{3}\right) \delta 0.74$ (d, J=6.6 Hz, $3 \mathrm{H}), 1.99(\mathrm{~m}, 2 \mathrm{H}), 2.42(\mathrm{~m}, 1 \mathrm{H}), 3.31(\mathrm{~s}, 3 \mathrm{H}), 3.88(\mathrm{~d}, J=6.2 \mathrm{~Hz}, 1 \mathrm{H}), 4.42(\mathrm{~d}, J=7.0$ $\mathrm{Hz}, 1 \mathrm{H}), 5.59$ (dt, $J=15.4,6.2 \mathrm{~Hz}, 1 \mathrm{H}), 5.72(\mathrm{dt}, J=15.4,7.3 \mathrm{~Hz}, 1 \mathrm{H}), 7.31(\mathrm{~m}, 5 \mathrm{H})$; HRMS, calcd for $\mathrm{C}_{14} \mathrm{H}_{20} \mathrm{O}_{2}: 220.1463$, found $m / z$ (relative intensity) $220.1441\left(\mathrm{M}^{+}, 4\right)$, 188 (10), 105 (100), 82 (49), 77 (21), 67 (22).

(2E)-5,6-anti-5-Metyl-6-phenyl-2-hexen-1,6-diol (4.5): IR (neat) 3380 (s), 3050 (m), 2980 (s), 2950 (s), 2890 (s), 1680 (w), 1500 (m), 1460 (s), 1350 (s), 1230 (s), 1090 (s), 1020 (s), 980 (s), 760 (s), 700 (s) cm ${ }^{-1} ;{ }^{1} \mathrm{H}$ NMR (400 MHz, C $\left.6 \mathrm{D}_{6}\right) \delta 0.71(\mathrm{~d}, J=7.0 \mathrm{~Hz}, 3 \mathrm{H}), 1.43$ (br s, $1 \mathrm{H}), 1.80$ (m, $1 \mathrm{H}), 1.95$ (br ddd, $J=5.9,8.4,12.8 \mathrm{~Hz}, 1 \mathrm{H}), 2.39$ (br dt, $J=12.8,4.2$ $\mathrm{Hz}, 1 \mathrm{H}), 3.83$ (d, $J=3.7 \mathrm{~Hz}, 2 \mathrm{H}), 4.14$ (d, $J=7.0 \mathrm{~Hz}, 1 \mathrm{H}), 5.46-5.57$ (m, $2 \mathrm{H}), 7.14-7.22$ (m, $5 \mathrm{H}$ ); HRMS, calcd for $\mathrm{C}_{13} \mathrm{H}_{18} \mathrm{O}_{2}$ : 206.1307, found $\mathrm{m} / z$ (relative intensity) 206.1260 $\left(\mathrm{M}^{+}, 2\right), 188$ (16), 144 (17), 134 (11), 107 (100).

1-(1-Allylcyclohexyl)-1-phenylmetanol (4.6): IR (neat) 3450 (s), 2940 (s), 1650 (w), 1500 (w), 1460 (s), 1350 (w), 1020 (m), 910 (m), 760 (m), 720 (m), 700 (s) cm-1; ${ }^{1}$ H NMR (400 $\left.\mathrm{MHz}, \mathrm{CDCl}_{3}\right) \delta 1.14-1.64(\mathrm{~m}, 10 \mathrm{H}), 2.15(\mathrm{dd}, J=7.5,14.5 \mathrm{~Hz}, 1 \mathrm{H}), 2.30$ (dd, $J=7.5$, $14.5 \mathrm{~Hz}, 1 \mathrm{H}), 4.64(\mathrm{~s}, 1 \mathrm{H}), 5.10$ (br d, $J=10.3 \mathrm{~Hz}, 1 \mathrm{H}), 5.11$ (br d, $J=16.9 \mathrm{~Hz}, 1 \mathrm{H})$, 5.97 (ddt, $J=10.3,16.9,7.5 \mathrm{~Hz}, 1 \mathrm{H}), 7.27-7.33(\mathrm{~m}, 5 \mathrm{H})$; HRMS, calcd for $\mathrm{C}_{16} \mathrm{H}_{22} \mathrm{O}$ : 230.1671, found $m / z$ (relative intensity) $230.1671\left(\mathrm{M}^{+}, 10\right), 189$ (3), 124 (35), 122 (100), 107 (71).

2,5-Dimethylhex-5-en-2-ol (4.7): IR (neat) 3441 (s), 2970 (s), 1643 (s), 1049 (s) cm-1; ${ }^{1} \mathrm{H}$ NMR (400 MHz, $\left.\mathrm{CDCl}_{3}\right) \delta 1.24(\mathrm{~s}, 6 \mathrm{H}), 1.62(\mathrm{dd}, J=5.5,7.9 \mathrm{~Hz}, 2 \mathrm{H}), 1.75(\mathrm{~s}, 3 \mathrm{H}), 2.09$ 
$(\mathrm{dd}, J=5.5,7.9 \mathrm{~Hz}, 2 \mathrm{H}), 4.71$ (br s, $2 \mathrm{H}) ;{ }^{13} \mathrm{C} \mathrm{NMR}\left(100 \mathrm{MHz}, \mathrm{CDCl}_{3}\right) \delta 22.6,29.2,32.6$, $41.7,70.9,109.6,146.1$.

1-(3-Methylbut-3-enyl)cyclohexanol (4.8): IR (neat) 3380 (s), 1650 (m), 1040 (m), 960 (s), $920(\mathrm{~s}) \mathrm{cm}^{-1} ;{ }^{1} \mathrm{H}$ NMR (400 MHz, $\left.\mathrm{CDCl}_{3}\right) \delta 1.40-1.63(\mathrm{~m}, 12 \mathrm{H}), 1.75(\mathrm{~s}, 3 \mathrm{H}), 2.06-2.14$ (m, $2 \mathrm{H}), 4.71$ (br s, $2 \mathrm{H}) ;{ }^{13} \mathrm{C}$ NMR (100 MHz, $\left.\mathrm{CDCl}_{3}\right) \delta 22.4,22.6,25.9,31.2,37.4,40.2$, 71.3, 109.5, 146.4; HRMS calcd for $\mathrm{C}_{11} \mathrm{H}_{20} \mathrm{O}$ : 168.1514, found $m / z$ (relative intensity) $168.1509\left(\mathrm{M}^{+}, 100\right)$.

6-Methoxy-2-Phenyl-2,3-dihydropyran-4-one (5): ${ }^{2}$ IR (neat) 1661 (s), 1582 (s), 1446 (s), 1398 (s), 1231 (s), 989 (s), 765 (m), 731 (m), 700 (s) cm ${ }^{-1} ;{ }^{1} \mathrm{H}$ NMR (400 MHz, CDCl 3 ) $\delta$ $2.62(\mathrm{dd}, J=3.1,16.9 \mathrm{~Hz}, 1 \mathrm{H}), 2.84(\mathrm{dd}, J=13.6,16.9 \mathrm{~Hz}, 1 \mathrm{H}), 3.82(\mathrm{~s}, 3 \mathrm{H}), 4.94(\mathrm{~s}, 1$ $\mathrm{H}), 5.51(\mathrm{dd}, J=3.1,13.6 \mathrm{~Hz}, 1 \mathrm{H}), 7.37-7.42(\mathrm{~m}, 5 \mathrm{H}) ;{ }^{13} \mathrm{C} \mathrm{NMR}\left(100 \mathrm{MHz}, \mathrm{CDCl}_{3}\right) \delta$ $42.2,55.9,81.5,82.8,126.1,128.9,137.4,174.1,191.8$.

2-Phenyl-4-methyltetrahedropyran (7.1): Into a solution of 2.2 (176.2 $\mathrm{mg}, 1 \mathrm{mmol}$, anti/syn $=15: 1)$ in THF $(10 \mathrm{~mL})$ was added diborane $(10 \mathrm{~mL}, 10 \mathrm{mmol} ; 1 \mathrm{M}$ THF solution $)$ dropwise at $0{ }^{\circ} \mathrm{C}$ for $15 \mathrm{~min}$ and the mixture was stirred at room temperature for $2 \mathrm{~h}$. To the mixture were added $30 \% \mathrm{H}_{2} \mathrm{O}_{2}(2 \mathrm{~mL})$ and $2 \mathrm{M} \mathrm{NaOH}(2 \mathrm{~mL})$ and stirred for $12 \mathrm{~h}$. The mixture was extracted with ethyl acetate $(2 \times 10 \mathrm{~mL})$. The organic phase was dried $\left(\mathrm{MgSO}_{4}\right)$ and concentrated in vacuo. To a solution of the residue in THF $(2 \mathrm{~mL})$, p-toluenesulfonyl chloride $(101 \mathrm{mg}, 0.53 \mathrm{mmol})$ and pyridine $(1 \mathrm{~mL})$ were added at $0{ }^{\circ} \mathrm{C}$ and the mixture was stirred for $5 \mathrm{~h}$. The mixture was diluted with EtOAc and washed with $2 \mathrm{M} \mathrm{HCl}(10 \mathrm{~mL})$ and with brine. The organic phase was dried $\left(\mathrm{MgSO}_{4}\right)$ and concentrated in vacuo. The residue was purified by column chromatography over silica gel (hexane-ethyl acetate, 8/1, v/v) to provide 7.1 (23\% overall) $(1,3-$ cis/1,3-trans $=4.6: 1)$. IR (neat) 2950 (s), 1450 (s), 1278 (s), 1090 (s), 750 (m), 700 (s) cm-1; ${ }^{1} \mathrm{H}$ NMR (400 MHz, $\mathrm{CDCl}_{3}, 1,3$-cis isomer): $\delta 0.98(\mathrm{~d} J=6.4 \mathrm{~Hz}, 3 \mathrm{H}), 1.24(\mathrm{dt}, J=12.8,11.2 \mathrm{~Hz}, 1 \mathrm{H}), 1.33$ 
(br dq, $J=4.8,12.8 \mathrm{~Hz}, 1 \mathrm{H}$ ), 1.61 (br ddd, $J=1.8,3.0,12.8 \mathrm{~Hz}, 1 \mathrm{H}$ ), 1.77 (m, $1 \mathrm{H}$, coalescing to $\mathrm{tt}, J=3.0,11.2 \mathrm{~Hz}$ by irradiation at 0.98$), 1.84(\mathrm{br} \mathrm{ddt}, J=3.0,12.8,2.0 \mathrm{~Hz}, 1$ H), $3.59(\mathrm{ddd}, J=2.0,11.2,12.8 \mathrm{~Hz}, 1 \mathrm{H}), 4.15(\mathrm{ddd}, J=2.0,4.8,11.2 \mathrm{~Hz}, 1 \mathrm{H}), 4.30$ (dd, $J=1.8,11.2 \mathrm{~Hz}, 1 \mathrm{H}), 7.20-7.39(\mathrm{~m}, 5 \mathrm{H}) ;{ }^{13} \mathrm{C} \mathrm{NMR}\left(100 \mathrm{MHz}, \mathrm{CDCl}_{3}, 1,3\right.$-cis isomer) $\delta$ 22.3, 30.8, 34.5, 42.7, 68.5, 79.9, 125.8, 127.3, 128.3, 143.3; ${ }^{13} \mathrm{C} \mathrm{NMR}\left(100 \mathrm{MHz}, \mathrm{CDCl}_{3}\right.$, 1,3-trans isomer) $\delta 18.6,25.4,32.1,38.9,63.5,74.0,126.1,127.1,128.3,142.9$; HRMS calcd for $\mathrm{C}_{12} \mathrm{H}_{16} \mathrm{O}: 176.1201$, found $\mathrm{m} / \mathrm{z}$ (relative intensity) $176.1191\left(\mathrm{M}^{+}, 100\right), 175$ (57), 105 (46), 91 (13), 77 (8).

trans- $\boldsymbol{\beta}$-Methyl- $\boldsymbol{\gamma}$-phenyl- $\boldsymbol{\gamma}$-butyrolactone (7.2): A solution of 4.2 (24.4 mg, $0.14 \mathrm{mmol}$, anti/syn $=5.5: 1)$ in $\mathrm{CH}_{2} \mathrm{Cl}_{2}(3 \mathrm{~mL})$ was cooled to $-78^{\circ} \mathrm{C}$, and ozone was bubbled through until a blue color appeared (ca. $10 \mathrm{~min}$ ). The excess of ozone was removed by a flow of $\mathrm{N}_{2}$. The mixture was allowed to warm to room temperature. Into this solution were added $\mathrm{AcOH}(1 \mathrm{~mL}), \mathrm{H}_{2} \mathrm{O}(0.1 \mathrm{~mL})$, conc. $\mathrm{H}_{2} \mathrm{SO}_{4}(0.1 \mathrm{~mL}), 30 \% \mathrm{H}_{2} \mathrm{O}_{2}(0.5 \mathrm{~mL})$ and this mixture was stirred at room temperature for $0.5 \mathrm{~h}$ and then heated at $60{ }^{\circ} \mathrm{C}$ for $4 \mathrm{~h}$. The mixture was diluted with ethyl acetate, washed with sat. $\mathrm{NaHCO}_{3}$ and with brine. The organic phase was dried $\left(\mathrm{MgSO}_{4}\right)$ and concentrated in vacuo. The residue was purified by column chromatography over silica gel (hexane-EtOAc, 8/1, v/v) to provide $7.2(60 \%$ overall). IR (neat) $3050(\mathrm{w}), 2950$ (m), 1780 (s), 1450 (m), 1270 (s), 1210 (s), 1140 (s), 1000 (s), $940(\mathrm{~m}), 750(\mathrm{~m}), 690(\mathrm{~s}) \mathrm{cm}^{-1} ;{ }^{1} \mathrm{H}$ NMR (400 MHz, $\left.\mathrm{CDCl}_{3}\right) \delta 1.20$ (d, J=6.2 Hz, $3 \mathrm{H}), 2.34(\mathrm{dd}, J=10.4,16.9 \mathrm{~Hz}, 1 \mathrm{H}), 2.48(\mathrm{~m}, 1 \mathrm{H}$, coalescing to dt, $J=10.4,8.1 \mathrm{~Hz}$ by irradiation at 1.20), $2.80(\mathrm{dd}, J=8.1,16.9 \mathrm{~Hz}, 1 \mathrm{H}), 4.94(\mathrm{~d}, J=8.1 \mathrm{~Hz}, 1 \mathrm{H}), 7.22-7.44(\mathrm{~m}$, $5 \mathrm{H}) ;{ }^{13} \mathrm{C} \mathrm{NMR}\left(100 \mathrm{MHz}, \mathrm{CDCl}_{3}\right)$ 4,5-trans isomer $: \delta$ 16.5, 37.2, 39.8, 125.9, 128.7, 138.0, 176.0; 4,5-cis isomer: $\delta$ 15.2, 30.8, 34.9, 125.4, 128.1, 138.0, 176.0; HRMS calcd for $\mathrm{C}_{11} \mathrm{H}_{12} \mathrm{O}_{2}: 176.0837$, found $m / z$ (relative intensity) $176.0838\left(\mathrm{M}^{+}, 85\right), 117$ (9), 107 (100), 105 (60), 77 (11). 
$\boldsymbol{\alpha}, \boldsymbol{\beta}$-Dimethyl- $\boldsymbol{\gamma}$-phenyl- $\boldsymbol{\gamma}$-butyrolactone (7.3): The same procedure for the synthesis of 7.2 was followed, and 7.3 (cis,cis/trans,trans $=5.3: 1$ ) was isolated in $60 \%$ overall yield from 2.9 (syn,anti/anti,anti = 8:1). IR (neat) $2975(\mathrm{~m}), 1780(\mathrm{~s}), 1454(\mathrm{~m}), 1180(\mathrm{~s}), 1055$ (s), $980(\mathrm{~m}), 704(\mathrm{~s}) \mathrm{cm}^{-1} ;{ }^{1} \mathrm{H}$ NMR (400 MHz, $\left.\mathrm{CDCl}_{3}\right)$ cis,cis-7.3 $\delta 0.55(\mathrm{~d}, J=7.2 \mathrm{~Hz}, 3$ $\mathrm{H}), 1.23(\mathrm{~d}, J=7.2 \mathrm{~Hz}, 3 \mathrm{H}$ ), 2.79 (dquint, $J=5.2,7.2 \mathrm{~Hz}, 1 \mathrm{H}$ ), 3.02 (quint, $J=7.2 \mathrm{~Hz}, 1$ $\mathrm{H}), 5.53(\mathrm{~d}, J=5.2 \mathrm{~Hz}, 1 \mathrm{H}), 7.22-7.43(\mathrm{~m}, 5 \mathrm{H}) ;{ }^{13} \mathrm{C} \mathrm{NMR}\left(100 \mathrm{MHz}, \mathrm{CDCl}_{3}\right) \delta 9.5,10.1$, 40.0, 44.1, 82.3, 125.3, 127.9, 128.5, 136.3, 178.9; trans,trans-7.3: ${ }^{1} \mathrm{H}$ NMR (400 MHz, $\left.\mathrm{CDCl}_{3}\right) \delta 1.15(\mathrm{~d}, J=7.2 \mathrm{~Hz}, 3 \mathrm{H}), 1.31(\mathrm{~d}, J=7.2 \mathrm{~Hz}, 3 \mathrm{H}), 2.00(\mathrm{ddq}, J=10.2,12.0,7.2$ $\mathrm{Hz}, 1 \mathrm{H}), 2.36(\mathrm{dq}, J=12.0,7.2 \mathrm{~Hz}, 1 \mathrm{H}), 4.82$ (d, $J=10.2 \mathrm{~Hz}, 1 \mathrm{H}), 7.22-7.43$ (m, $5 \mathrm{H})$; ${ }^{13} \mathrm{C}$ NMR $\left(100 \mathrm{MHz}, \mathrm{CDCl}_{3}\right) \delta 12.9,14.4,43.4,47.9,86.2,126.3,128.7,128.8,137.6$, 178.5; HRMS calcd for $\mathrm{C}_{12} \mathrm{H}_{14} \mathrm{O}_{2}: 190.0994$, found $\mathrm{m} / z$ (relative intensity) 190.1003 (M+, 40), 146 (9), 107 (100), 91 (5), 77 (10).

cis-5-((2E)-Butenyl)-2,2-dimethyl-4-phenyl-1,3-dioxane (7.4): Into a solution of 2.12 $(123.3 \mathrm{mg}, 0.53 \mathrm{mmol})$ in THF $(3 \mathrm{~mL})$ was added $\mathrm{LiAlH}_{4}(25 \mathrm{mg}, 0.66 \mathrm{mg})$ at $0{ }^{\circ} \mathrm{C}$. After stirring at the same temperature for $4 \mathrm{~h}$ and then at room temperature $2 \mathrm{~h}$, the mixture was quenched by addition of a mixture of $\mathrm{THF} / \mathrm{H}_{2} \mathrm{O}(5: 1,6 \mathrm{~mL})$ and then sat. $\mathrm{NH}_{4} \mathrm{Cl}(30$ $\mathrm{mL})$. The mixture was extracted with ethyl acetate $(2 \times 20 \mathrm{~mL})$, and the combined extracts were washed with brine, dried $\left(\mathrm{MgSO}_{4}\right)$. The solvent was removed in vacuo, and the residue was purified by flash chromatography over silica gel (hexane/EtOAc, 8/1, v/v) to provide 2-hydroxymethyl-1-phenyl-4-hexenol (75\%). A solution of the diol (77.9 $\mathrm{mg}$, $0.38 \mathrm{mmol})$ and $p$-toluenesulfonic acid monohydrate $(10 \mathrm{mg}, 0.05 \mathrm{mmol})$ in acetone dimethyl acetal ( $3 \mathrm{~mL}, 24.4 \mathrm{mmol}$ ) was stirred at room temperature for $1 \mathrm{~h}$. After dilution with ethyl acetate $(10 \mathrm{~mL})$, the mixture was washed with sat. $\mathrm{NaHCO}_{3}$ and brine. The organic phase was dried $\left(\mathrm{MgSO}_{4}\right)$ and concentrated in vacuo to give 7.4 (87\%): IR (neat) 2930 (s), 2860 (s), 1710 (w), 1640 (w), 1580 (w), 1510 (m), 1460 (m), 1380 (m), 1300 (m), 
$1240(\mathrm{~m}), 1050(\mathrm{~s}), 1010(\mathrm{~m}), 880(\mathrm{~s}), 670(\mathrm{~s}) \mathrm{cm}^{-1} ;{ }^{1} \mathrm{H}$ NMR (400 MHz, $\left.\mathrm{C}_{6} \mathrm{D}_{6}\right) \delta 1.32(\mathrm{~m}$, $1 \mathrm{H}$, coalescing to $\mathrm{dm}, J=9.9 \mathrm{~Hz}$ by irradiation at 1.80$), 1.34(\mathrm{~s}, 3 \mathrm{H}), 1.48(\mathrm{br} \mathrm{d}, J=6.2$ $\mathrm{Hz}, 3 \mathrm{H}), 1.60(\mathrm{~s}, 3 \mathrm{H}), 1.80(\mathrm{~m}, 1 \mathrm{H}$, coalescing to br $\mathrm{d}, J=13.4 \mathrm{~Hz}$ by irradiation at 5.14), $2.54(\mathrm{~m}, 1 \mathrm{H}$, coalescing to $\mathrm{dd} J=8.6,9.9 \mathrm{~Hz}$ by irradiation at 1.80$), 3.84(\mathrm{dm}, J=11.5 \mathrm{~Hz}$, coalescing to dd, $J=2.6,11.5 \mathrm{~Hz}$ by irradiation at 1.80$), 3.90(\mathrm{dd}, J=1.4,11.5 \mathrm{~Hz}, 1 \mathrm{H})$, $5.01(\mathrm{~d}, J=2.6 \mathrm{~Hz}, 1 \mathrm{H}), 5.14(\mathrm{~m}, 1 \mathrm{H}$, coalescing to ddd, $J=5.9,8.6,15.0 \mathrm{~Hz}$ by irradiation at 1.48), $5.32(\mathrm{~m}, 1 \mathrm{H}$, coalescing to $\mathrm{d}, J=15.0 \mathrm{~Hz}$ by irradiation at 1.48$)$, 7.05-7.35 (m, $5 \mathrm{H}$ ); HRMS calcd for $\mathrm{C}_{16} \mathrm{H}_{22} \mathrm{O}_{2}$ : 246.1620, found $m / z$ (relative intensity) $246.1628\left(\mathrm{M}^{+}, 18\right), 188(62), 165$ (39), 107 (100), 67 (63).

trans-2,2-Dimethyl-4-phenyl-6-vinyl-1,3-dioxane (7.5): Into a solution of $2.16(287.2 \mathrm{mg}$, $0.86 \mathrm{mmol})$ in THF $(4 \mathrm{~mL})$ was added $\mathrm{Bu}_{4} \mathrm{~N}^{+} \mathrm{F}^{-}(1 \mathrm{~mL}, 1 \mathrm{M} \mathrm{THF})$ at room temperature. After stirring for $1 \mathrm{~h}$, the mixture was diluted with ethyl acetate $(20 \mathrm{~mL})$ and washed with brine. The organic phase was dried $\left(\mathrm{MgSO}_{4}\right)$ and concentrated in vacuo. The residue was purified by column chromatography over silica gel (hexane/ethyl acetate, 2/1, v/v) to provide anit-1-phenyl-4-penten-1,3-diol in 90\% yield. A solution of the diol $(28.5 \mathrm{mg}$, $0.16 \mathrm{mmol})$ and $p$-toluenesulfonic acid monohydrate $(4 \mathrm{mg}, 0.02 \mathrm{mmol})$ in 2,2-dimethoxypropane $(3 \mathrm{~mL}, 24.4 \mathrm{mmol})$ was stirred at room temperature for $1 \mathrm{~h}$. After dilution with ethyl acetate $(10 \mathrm{~mL})$, the mixture was washed with sat. $\mathrm{NaHCO}_{3}$ and brine. The organic phase was dried $\left(\mathrm{MgSO}_{4}\right)$ and concentrated in vacuo to give $7.5(87 \%)$. IR (neat) $2940(\mathrm{~s}), 2910(\mathrm{~s}), 1650(\mathrm{w}), 1600(\mathrm{w}), 1450(\mathrm{~s}), 1280$ (s), 1120 (s), 1060 (s), $920(\mathrm{~s})$, 750 (s), 700 (s) cm ${ }^{-1} ;{ }^{1} \mathrm{H}$ NMR (400 MHz, $\mathrm{CDCl}_{3}$ ) $\delta 1.48$ (s $\left.3 \mathrm{H}\right), 1.49$ (s, $\left.3 \mathrm{H}\right), 2.07$ (dd, J $=7.5,7.7 \mathrm{~Hz}, 2 \mathrm{H}), 4.51(\mathrm{br} \mathrm{dt}, J=6.0,7.5 \mathrm{~Hz}, 1 \mathrm{H}$, coalescing to br $\mathrm{t}, J=7.5 \mathrm{~Hz}$ by irradiation at 5.97), $4.93(\mathrm{t}, J=7.7 \mathrm{~Hz}, 1 \mathrm{H}), 5.17(\mathrm{dd}, J=1.5,10.4 \mathrm{~Hz}, 1 \mathrm{H}), 5.27(\mathrm{dd}, J=$ 1.5, 17.2 Hz, $1 \mathrm{H}), 5.97$ (ddd, $J=6.0,10.4,17.2 \mathrm{~Hz}, 1 \mathrm{H}), 7.21-7.43$ (m, $5 \mathrm{H})$; HRMS calcd for $\mathrm{C}_{14} \mathrm{H}_{18} \mathrm{O}_{2}: 218.1307$, found $\mathrm{m} / z$. (relative intensity) $218.1367\left(\mathrm{M}^{+}, 12\right), 203(72)$, 
160 (90), 143 (90), 104 (100).

2,2,4-Trimethyl-5-(2-methylbut-3-enyl)-1,3-dioxolane (7.6): Into a solution of 2.38 (129 $\mathrm{mg}, 0.5 \mathrm{mmol})$ in THF $(4 \mathrm{~mL})$ was added $\mathrm{Bu}_{4} \mathrm{~N}^{+} \mathrm{F}^{-}(1 \mathrm{~mL}, 1 \mathrm{mmol}$ in $1 \mathrm{M} \mathrm{THF})$ at room temperature. After stirring for $2 \mathrm{~h}$, the mixture was diluted with ethyl acetate $(20 \mathrm{~mL})$ and washed with brine. The organic phase was dried $\left(\mathrm{MgSO}_{4}\right)$ and concentrated in vacuo to give a colorless oil. Into a solution of the residual oil in 2,2-dimethoxypropane (5 mL, 40 mmol) was added $p$-toluenesulfonic acid monohydrate $(10 \mathrm{mg}, 0.05 \mathrm{mmol})$ and the reaction mixture was stirred at room temperature for $12 \mathrm{~h}$. After dilution with ethyl acetate (10 $\mathrm{mL}$ ), the mixture was washed with sat. $\mathrm{NaHCO}_{3}$ and brine. The organic phase was dried $\left(\mathrm{MgSO}_{4}\right)$ and concentrated in vacuo, and the residue was purified by column chromatography over silica gel (hexane/ethyl acetate $=12 / 1, \mathrm{v} / \mathrm{v}$ ) to give 1,3-dioxolane 7.6 in $89 \%$ yield as a mixture of a 1:1 ratio. IR (neat) $2934(\mathrm{~s}), 1641(\mathrm{~m}), 1094(\mathrm{~s}) \mathrm{cm}^{-1} ;{ }^{1} \mathrm{H}$ NMR (400 MHz, $\mathrm{CDCl}_{3}$, one isomer) $\delta 1.05(\mathrm{~d}, J=6.2 \mathrm{~Hz}, 3 \mathrm{H}), 1.14(\mathrm{~d}, J=6.2 \mathrm{~Hz}, 3 \mathrm{H})$, $1.33(\mathrm{~s}, 3 \mathrm{H}), 1.38(\mathrm{~s}, 3 \mathrm{H}), 1.64(\mathrm{ddm}, J=4.3,14.1 \mathrm{~Hz}, 1 \mathrm{H}), 1.67$ (ddm, $J=8.3,14.1 \mathrm{~Hz}$, $1 \mathrm{H}), 2.31(\mathrm{dqm}, J=7.1,6.2 \mathrm{~Hz}, 1 \mathrm{H}), 4.12(\mathrm{dt}, J=8.3,6.2 \mathrm{~Hz}, 1 \mathrm{H}), 4.23$ (quint, $J=6.2$ $\mathrm{Hz}, 1 \mathrm{H}), 4.93$ (br d, $J=10.2 \mathrm{~Hz}, 1 \mathrm{H}), 5.00$ (br d, $J=16.9 \mathrm{~Hz}, 1 \mathrm{H}), 5.78$ (ddd, $J=7.1$, 10.2, $16.9 \mathrm{~Hz}, 1 \mathrm{H}) ;{ }^{13} \mathrm{C} \mathrm{NMR}\left(100 \mathrm{MHz}, \mathrm{CDCl}_{3}\right.$, one isomer) $\delta 15.6,19.3,26.0,27.3$, 34.2, 36.2, 75.6, 80.1, 107.3, 112.5, 144.6; ${ }^{1} \mathrm{H} \mathrm{NMR}\left(400 \mathrm{MHz}, \mathrm{CDCl}_{3}\right.$, the other isomer) $\delta$ $1.05(\mathrm{~d}, J=6.2 \mathrm{~Hz}, 3 \mathrm{H}), 1.25(\mathrm{~d}, J=6.2 \mathrm{~Hz}, 3 \mathrm{H}), 1.39$ (s, $3 \mathrm{H}), 1.44(\mathrm{~s}, 3 \mathrm{H}), 1.63$ (ddm, $J$ $=8.3,14.1 \mathrm{~Hz}, 1 \mathrm{H}), 1.66(\mathrm{ddm}, J=6.2,14.1 \mathrm{~Hz}, 1 \mathrm{H}), 2.38(\mathrm{dqm}, J=7.1,6.2 \mathrm{~Hz}, 1 \mathrm{H})$, $3.60(\mathrm{dt}, J=4.3,8.3 \mathrm{~Hz}, 1 \mathrm{H}), 3.71(\mathrm{dq}, J=8.3,6.2 \mathrm{~Hz}, 1 \mathrm{H}), 4.93(\mathrm{br} \mathrm{d}, J=10.2 \mathrm{~Hz}, 1 \mathrm{H})$, 5.00 (br d, $J=16.9 \mathrm{~Hz}, 1 \mathrm{H}), 5.78(\mathrm{ddd}, J=7.1,10.2,16.9 \mathrm{~Hz}, 1 \mathrm{H}) ;{ }^{13} \mathrm{C} \mathrm{NMR}(100 \mathrm{MHz}$, $\mathrm{CDCl}_{3}$, the other isomer) $\delta 17.6,19.5,27.3,28.6,34.2,39.2,73.8,77.3,107.7,112.5$, 144.4; HRMS, calcd for $\mathrm{C}_{11} \mathrm{H}_{20} \mathrm{O}_{2}-\mathrm{CH}_{3}$ : 169.1229, found $\mathrm{m} / z$ (relative intensity) $169.1229\left(\mathrm{M}^{+}-\mathrm{CH}_{3}, 37\right), 116$ (11), 115 (100), 109 (30), 107 (23). 
$\boldsymbol{\alpha}$-((E)-2-Butenyl)- $\boldsymbol{\beta}$-hydroxy- $\boldsymbol{\gamma}$-methyl- $\boldsymbol{\gamma}$-butyrolactone (7.7): Into a round-bottom flask was placed $2.40(158 \mathrm{mg}, 0.5 \mathrm{mmol})$ and a solution of trifluoroacetic acid (15 mL, TFA/THF/water $=1: 1: 1, \mathrm{v} / \mathrm{v}$ ) was introduced via a syringe. The reaction mixture was stirred at room temperature for $1 \mathrm{~h}$. The reaction mixture was diluted with ether $(15 \mathrm{~mL})$ and was washed with sat. $\mathrm{NaHCO}_{3}$ and brine. The organic phase was dried $\left(\mathrm{MgSO}_{4}\right)$ and concentrated in vacuo. The residue was purified by means of column chromatography over silica gel (hexane/ethyl acetate $=12 / 1, \mathrm{v} / \mathrm{v}$ ) to give $\gamma$-butyrolactone 7.7 in $89 \%$ as a mixture of diastereomers in a 1:1 ratio. IR (neat) 3439 (s), 1759 (s), $1059(\mathrm{~s}) \mathrm{cm}^{-1} ;{ }^{1} \mathrm{H}$ NMR (400 MHz, $\mathrm{CDCl}_{3}$, one isomer) $\delta 1.39(\mathrm{~d}, J=6.3 \mathrm{~Hz}, 3 \mathrm{H}), 1.68(\mathrm{~d}, J=6.3 \mathrm{~Hz}, 3 \mathrm{H})$, $2.29(\mathrm{~m}, 1 \mathrm{H}), 2.45-2.68(\mathrm{~m}, 2 \mathrm{H}), 3.85(\mathrm{dd}, J=4.6,13.1 \mathrm{~Hz}, 1 \mathrm{H}), 4.63(\mathrm{dq}, J=13.1,6.3$ $\mathrm{Hz}, 1 \mathrm{H}), 5.51(\mathrm{dm}, J=14.4 \mathrm{~Hz}, 1 \mathrm{H}), 5.62(\mathrm{dq}, J=14.4,6.3 \mathrm{~Hz}, 1 \mathrm{H}) ;{ }^{13} \mathrm{C}$ NMR $(100$ $\mathrm{MHz}, \mathrm{CDCl}_{3}$, one isomer) $\delta 17.9,31.2,48.8,73.1,77.4,78.2,126.6,129.2,175.3 ;{ }^{1} \mathrm{H}$ NMR (400 MHz, $\mathrm{CDCl}_{3}$, the other isomer) $\delta 1.45(\mathrm{~d}, J=6.3 \mathrm{~Hz}, 3 \mathrm{H}), 1.68(\mathrm{~d}, J=6.3 \mathrm{~Hz}$, $3 \mathrm{H}), 2.29(\mathrm{~m}, 1 \mathrm{H}), 2.45-2.68(\mathrm{~m}, 2 \mathrm{H}), 4.18-4.27(\mathrm{~m}, 2 \mathrm{H}), 5.51(\mathrm{dm}, J=14.4 \mathrm{~Hz}, 1 \mathrm{H})$, $5.62(\mathrm{dq}, J=14.4,6.3 \mathrm{~Hz}, 1 \mathrm{H}) ;{ }^{13} \mathrm{C} \mathrm{NMR}\left(100 \mathrm{MHz}, \mathrm{CDCl}_{3}\right.$, the other isomer) $\delta 18.2$, 31.2, 48.8, 73.1, 78.1, 79.7, 126.5, 129.2, 177.0; HRMS, calcd for $\mathrm{C}_{9} \mathrm{H}_{14} \mathrm{O}_{3}: 170.0943$, found $m / z$ (relative intensity) $170.0957\left(\mathrm{M}^{+}, 100\right), 153(2), 152(5), 141$ (4).

5-(6,6-Dimethyl-5,7-dioxaoctyl)-2,2-dimethyl-4-phenyl-1,3-dioxane (7.8): A solution of 1-(2-cyclohexenyl)-1-phenylmethanol (2.46) (225 mg, $1.2 \mathrm{mmol})$ in dichloromethane (10 $\mathrm{mL}$ ) was cooled to $-78{ }^{\circ} \mathrm{C}$, and ozone was bubbled through for $20 \mathrm{~min}$ until a blue color appeared. The excess of ozone was removed by a flow of nitrogen and the solvent was removed by a rotary evaporator. The residue was dissolved in $\mathrm{EtOH}(3 \mathrm{~mL})$ and treated with $\mathrm{NaBH}_{4}(152 \mathrm{mg}, 4 \mathrm{mmol})$ at $0{ }^{\circ} \mathrm{C}$ and then at room temperature for $6 \mathrm{~h}$. The reaction mixture was concentrated in vacuo and the residue was diluted with ethyl acetate $(30 \mathrm{~mL})$ and washed with $2 \mathrm{M} \mathrm{HCl}$, sat. $\mathrm{NaHCO}_{3}$, and brine. The organic phase was dried $\left(\mathrm{MgSO}_{4}\right)$ and 
concentrated in vacuo. Into a solution of the residue dissolved in 2,2-dimethoxypropane (10 $\mathrm{mL}, 80 \mathrm{mmol}$ ) was added $p$-toluenesulfonic acid monohydrate $(20 \mathrm{mg}, 0.1 \mathrm{mmol})$, and the reaction mixture was stirred at room temperature for $12 \mathrm{~h}$. After dilution with ethyl acetate $(10 \mathrm{~mL})$, the mixture was washed with sat. $\mathrm{NaHCO}_{3}$ and brine. The organic phase was dried $\left(\mathrm{MgSO}_{4}\right)$ and concentrated in vacuo. The residue was purified by means of column chromatography over silica gel (hexane/ethyl acetate $=12 / 1, \mathrm{v} / \mathrm{v}$ ) to provide 1,3-dioxane 7.8 in 60\% yield as a mixture of cis and trans $=4: 1 . \quad$ IR (neat) 2939 (s), 1049 (s), $849(\mathrm{~m}), 702(\mathrm{~m}) \mathrm{cm}^{-1} ;{ }^{1} \mathrm{H} \mathrm{NMR}\left(400 \mathrm{MHz}, \mathrm{CDCl}_{3}\right.$, cis isomer) $\delta 0.96-1.41(\mathrm{~m}, 6 \mathrm{H})$, 1.27 (s, $6 \mathrm{H}), 1.51$ (s, $3 \mathrm{H}), 1.54$ (s, $3 \mathrm{H}), 1.64$ (dm, $J=2.5 \mathrm{~Hz}, 1 \mathrm{H}), 3.12$ (s, $3 \mathrm{H}), 3.23$ (t, $J$ $=6.0 \mathrm{~Hz}, 2 \mathrm{H}), 3.88(\operatorname{brd}, J=11.7 \mathrm{~Hz}, 1 \mathrm{H}), 4.18(\mathrm{br} \mathrm{d}, J=11.7 \mathrm{~Hz}, 1 \mathrm{H}), 5.18(\mathrm{~d}, J=2.5$ $\mathrm{Hz}, 1 \mathrm{H}), 7.22-7.34(\mathrm{~m}, 5 \mathrm{H}) ;{ }^{13} \mathrm{C} \mathrm{NMR}\left(100 \mathrm{MHz}, \mathrm{CDCl}_{3}\right.$, cis isomer) $\delta$ 19.0, 23.1, 24.2, $24.4,29.7,29.9,39.2,48.3,60.3,63.0,73.4,98.9,99.5,125.3,126.6,127.9,140.7 ;{ }^{1} \mathrm{H}$ NMR (400 MHz, $\mathrm{CDCl}_{3}$, trans isomer) $\delta 0.96-1.41(\mathrm{~m}, 6 \mathrm{H}), 1.27(\mathrm{~s}, 6 \mathrm{H}), 1.55(\mathrm{~s}, 3 \mathrm{H})$, 1.56 (s, $3 \mathrm{H}), 1.89$ (ddm, $J=4.9,10.2 \mathrm{~Hz}, 1 \mathrm{H}), 3.09$ (s, $3 \mathrm{H}), 3.23$ (t, $J=6.0 \mathrm{~Hz}, 2 \mathrm{H}), 3.72$ $(\mathrm{t}, J=11.5 \mathrm{~Hz}, 1 \mathrm{H}), 3.97(\mathrm{dd}, J=4.9,11.5 \mathrm{~Hz}, 1 \mathrm{H}), 4.46(\mathrm{~d}, J=10.2 \mathrm{~Hz}, 1 \mathrm{H}), 7.22-7.34$ $(\mathrm{m}, 5 \mathrm{H}) ;{ }^{13} \mathrm{C} \mathrm{NMR}\left(100 \mathrm{MHz}, \mathrm{CDCl}_{3}\right.$, trans isomer) $\delta 19.1,23.1,24.2,24.4,27.6,29.8$, 40.6, 48.3, 60.1, 64.8, 77.8, 98.5, 99.6, 127.5, 127.9, 128.2, 140.2. Anal. calcd for $\mathrm{C}_{20} \mathrm{H}_{32} \mathrm{O}_{4}$ : C, 71.39; H, 9.59, found: C, 71.27; H, 9.78.

(1) (a) Müller, S. N.; Batra, R.; Senn, M.; Giese, B.; Kisel, M.; Shadyro, O. J. Am. Chem. Soc. 1997, 119, 2795-2803; (b) Harmata, M.; Sharma, U. Org. Lett. 2000, 2, 2703-2705.

(2) Danishefsky, M.; Harvey, D. F.; Quallich, G.; Uang, B. J. J. Org. Chem. 1984, 49, 393. 
NMR Spectra 


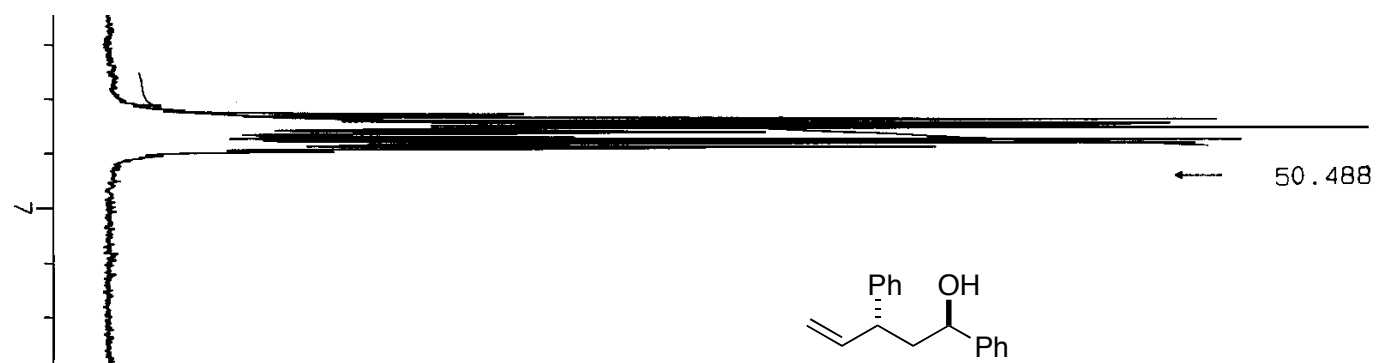

anti-1,3-Diphenyl-4-pentenol (2.4)

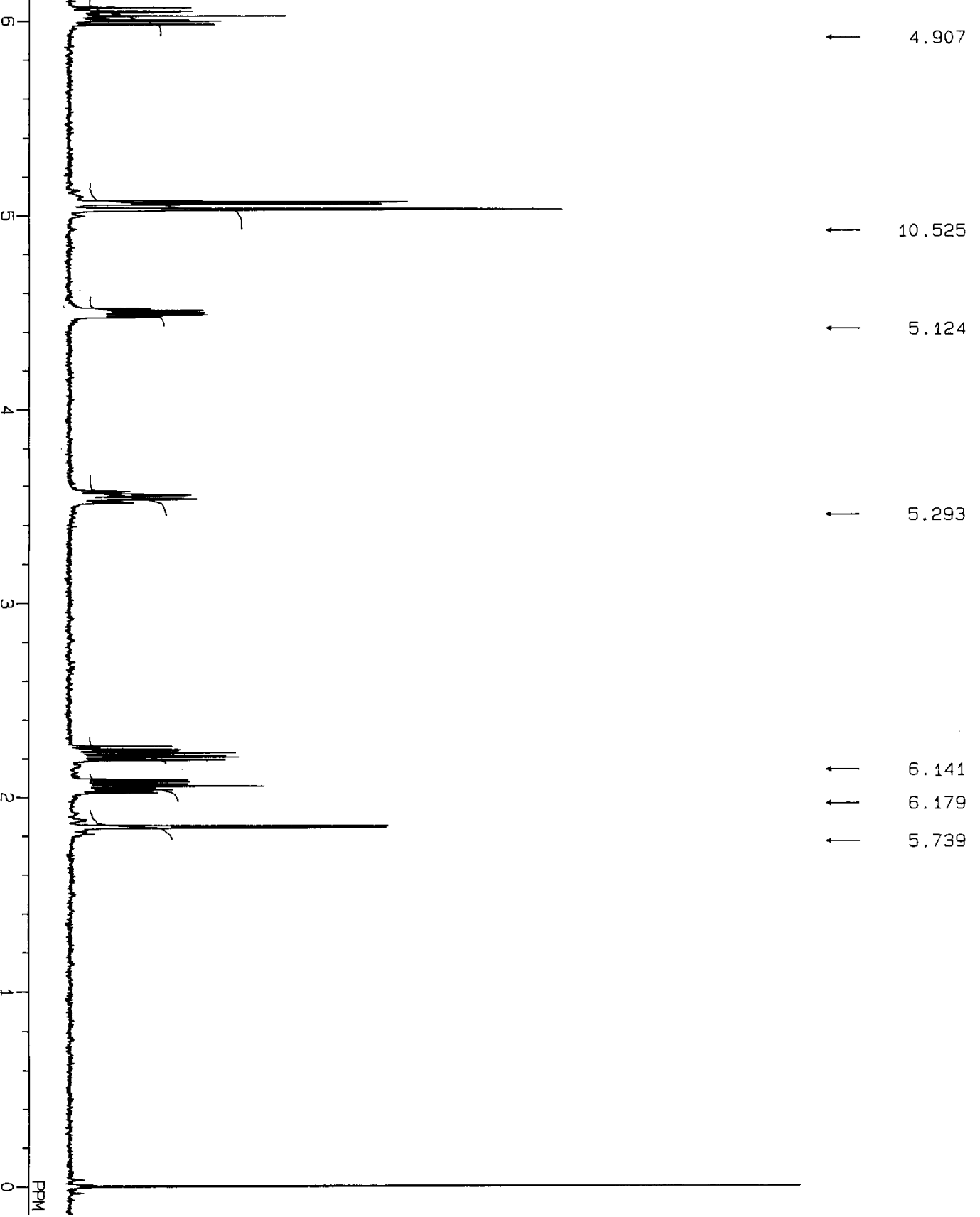




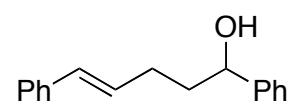

(4E)-1,5-Diphenyl-4-pentenol (2.7)

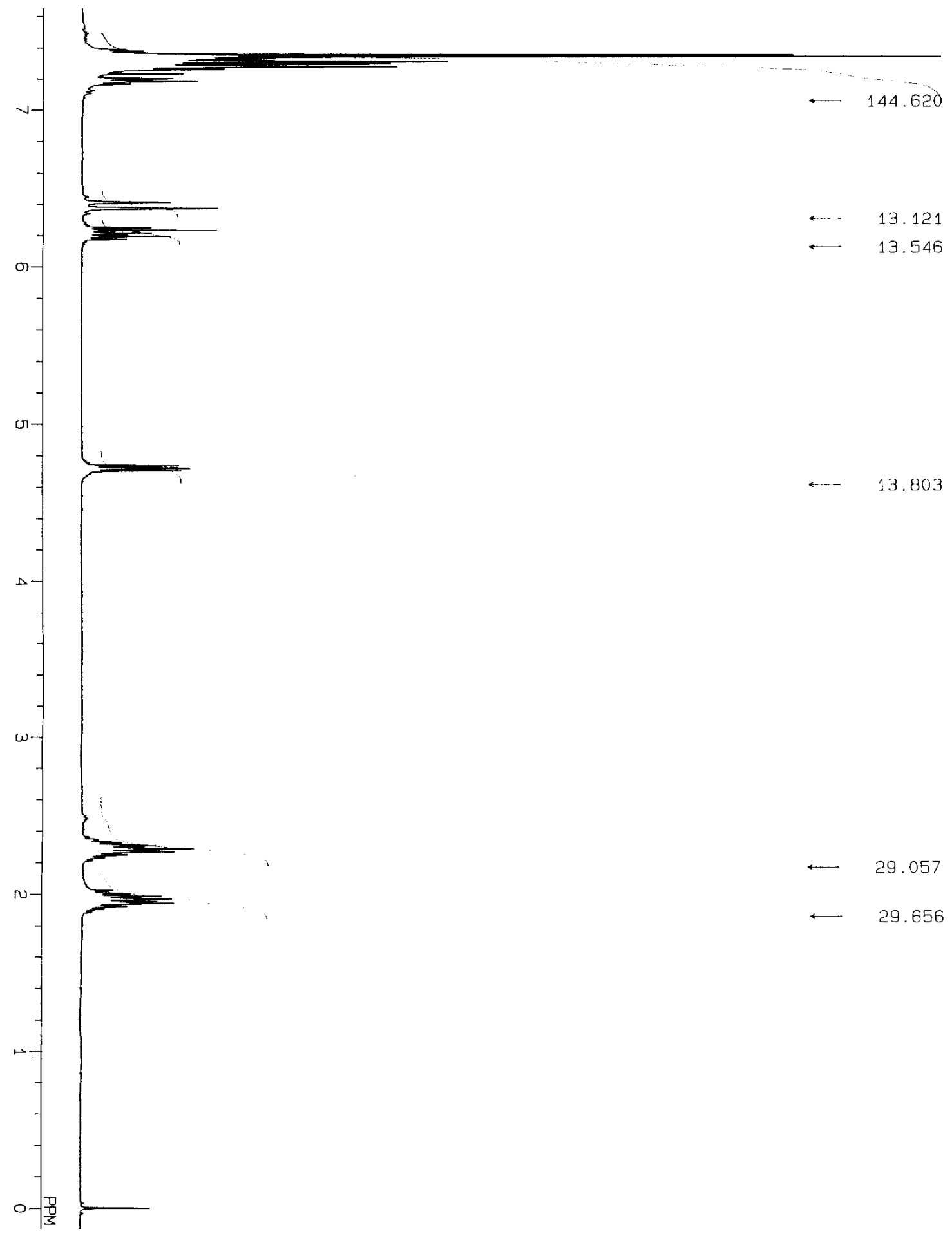




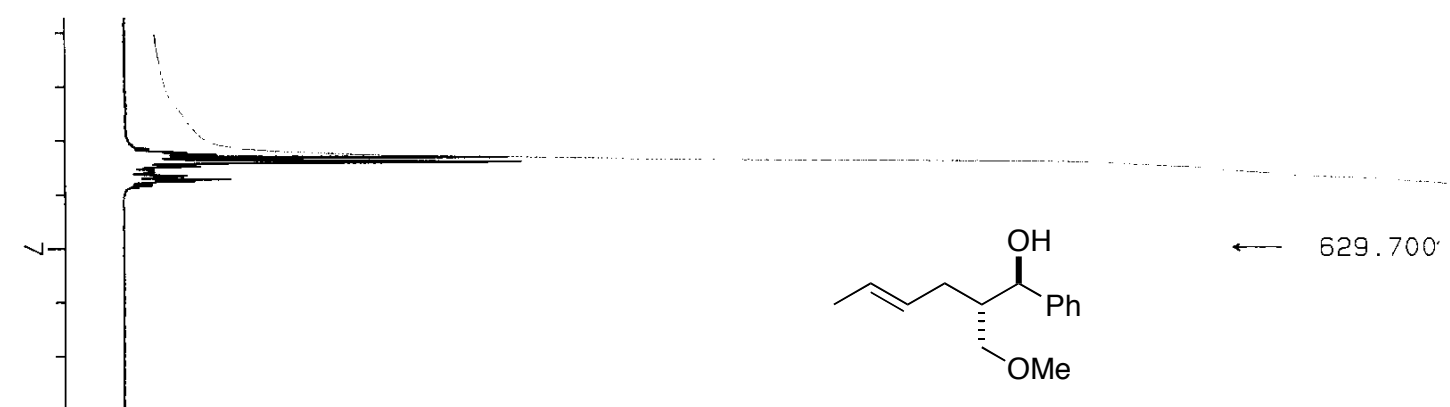

(4E)-1,2-anti-1-Phenyl-2-Methoxymethyl-4-hexen-1-ol (2.13)

$\sigma$

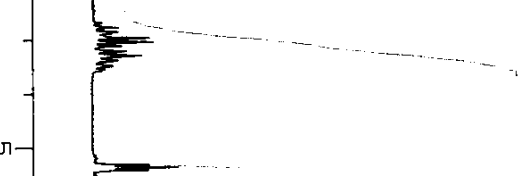

$\longleftarrow \quad 193.850$

$\longleftarrow \quad 92.850$

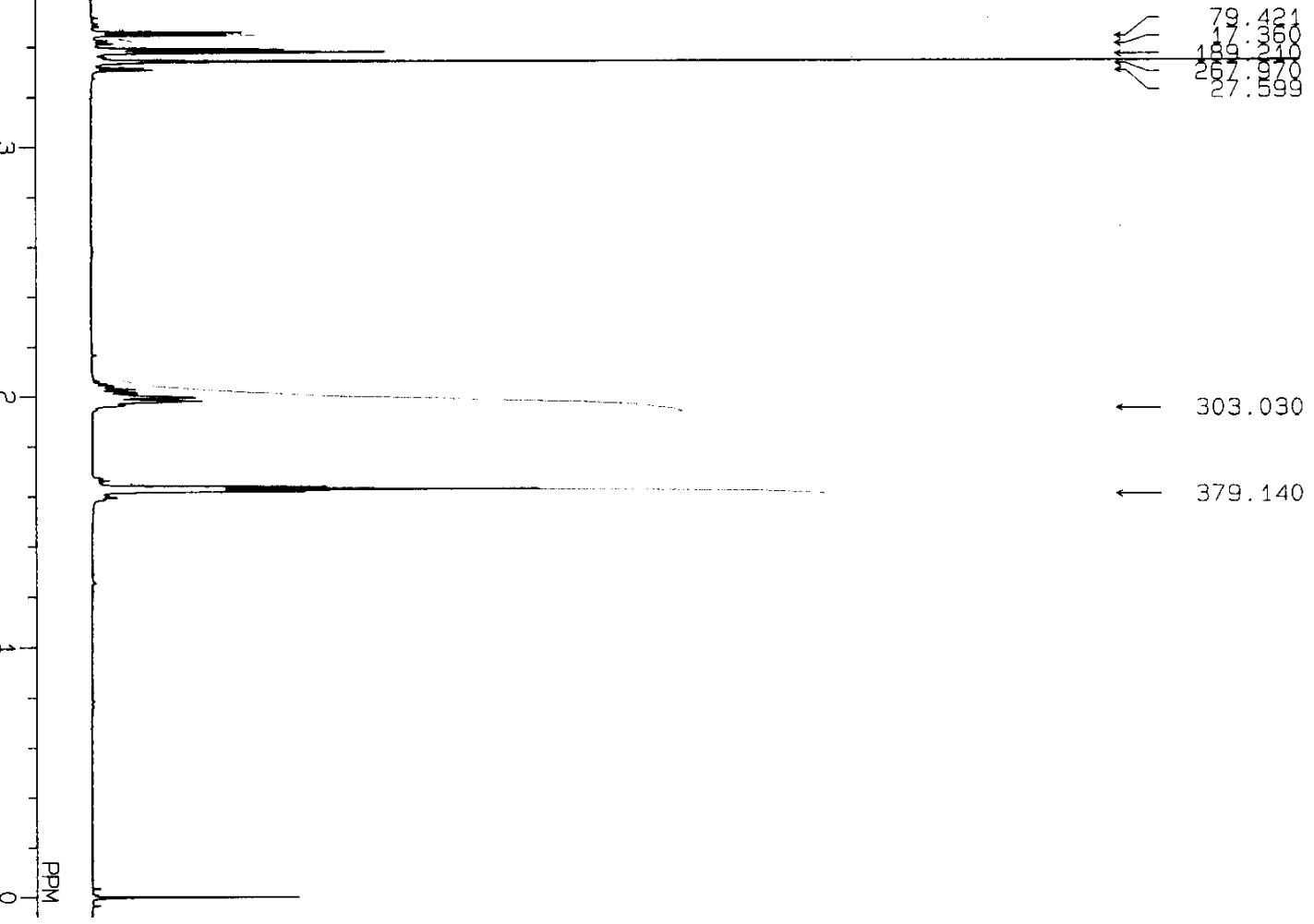




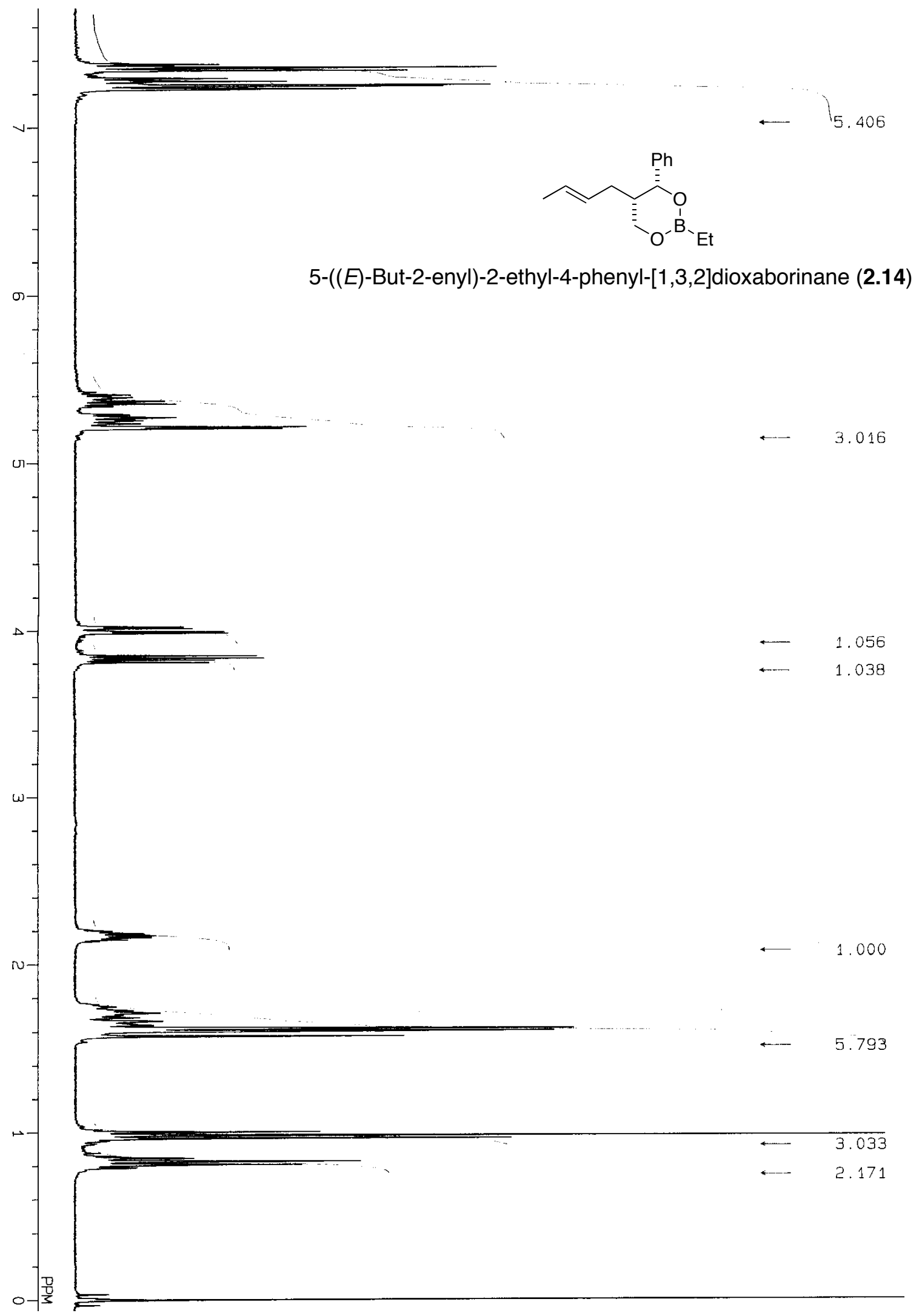




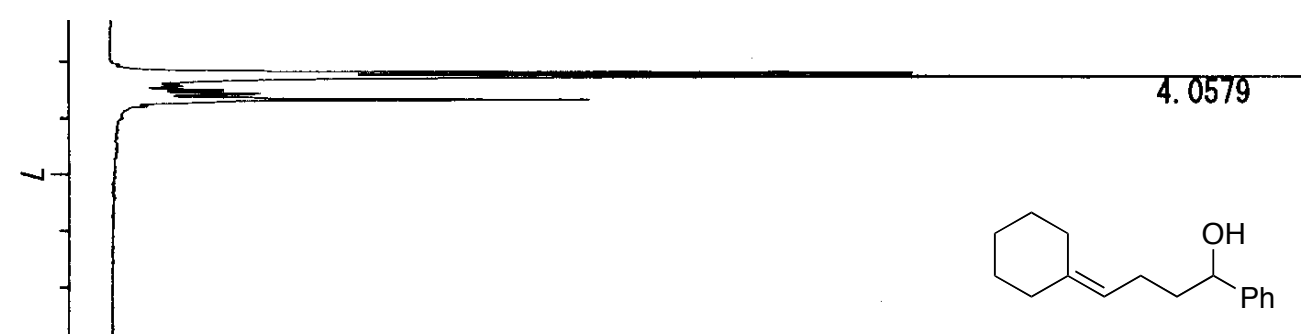

4-Cyclohexylidene-1-phenylbutan-1-ol (2.15)

$$
1.0
$$

1. 042

5.082

2. 8562

6. 3121 


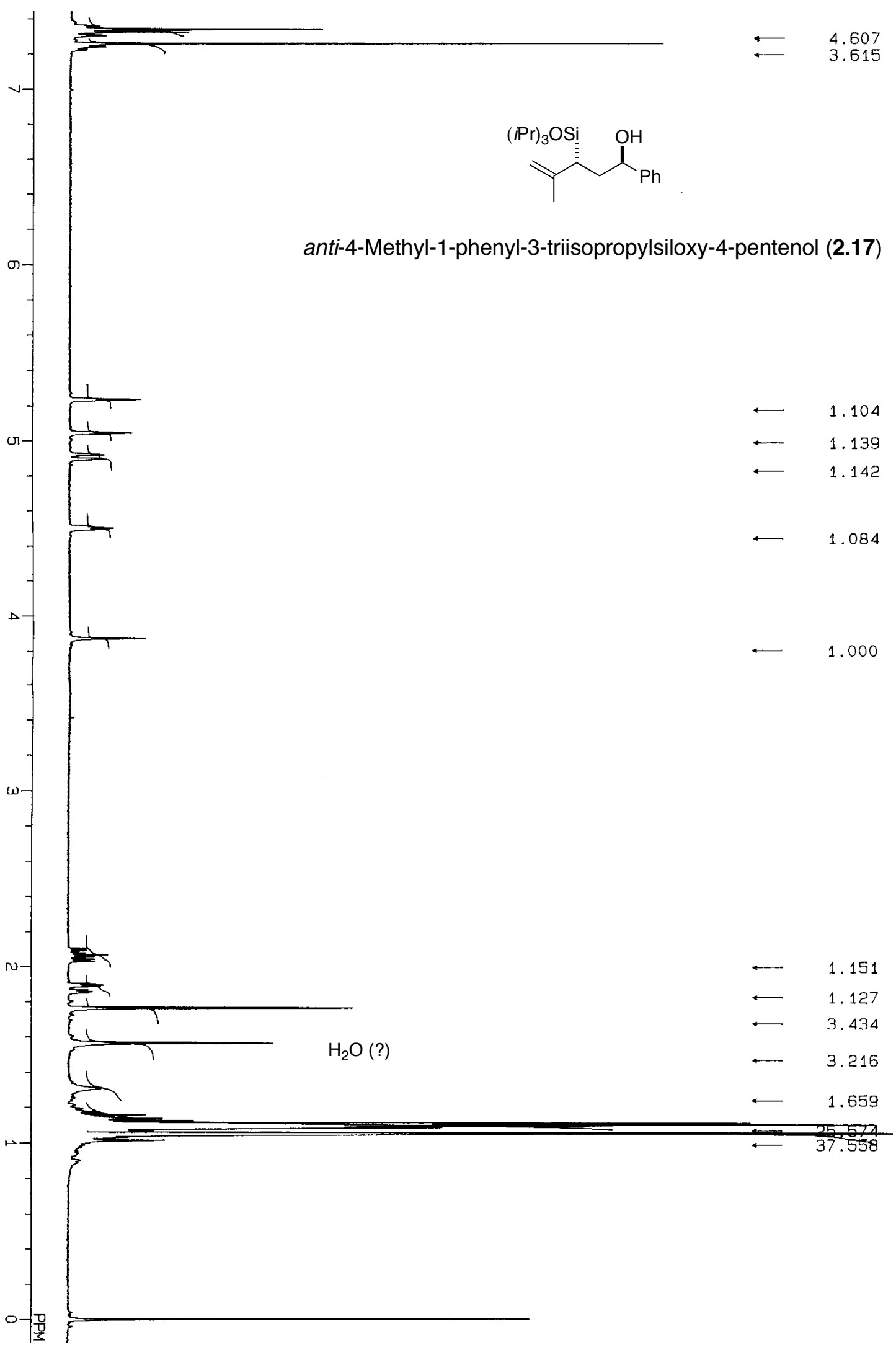




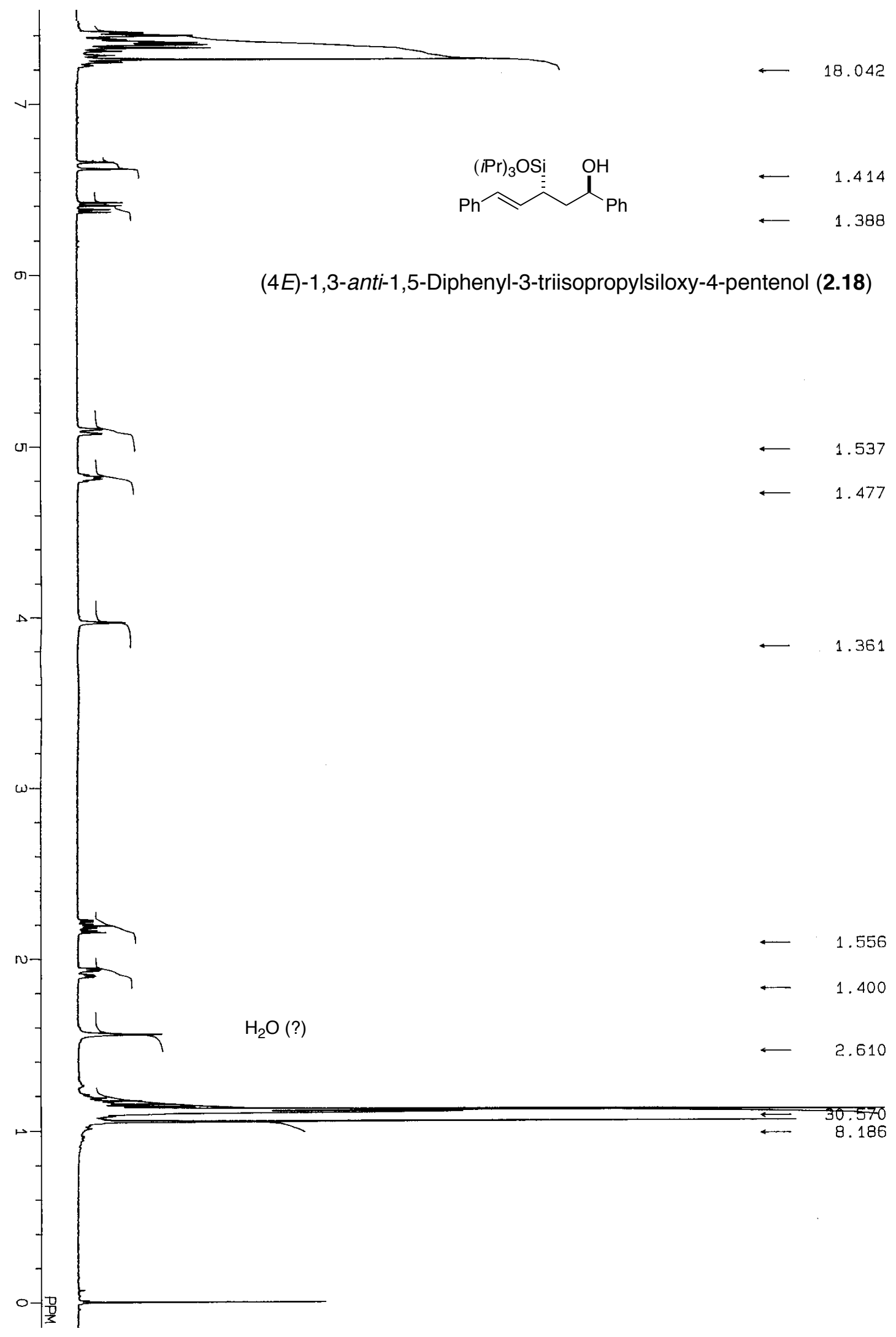




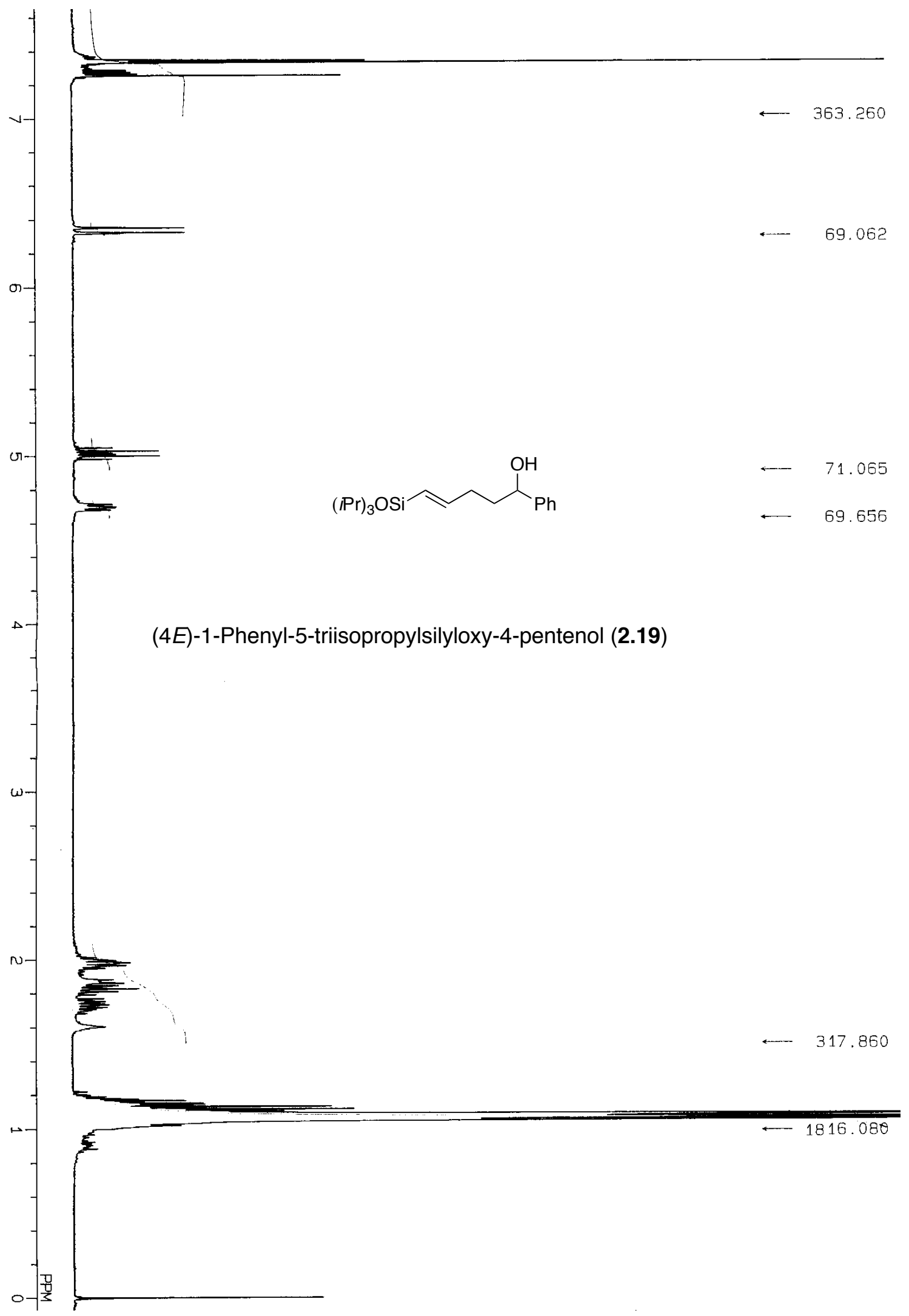




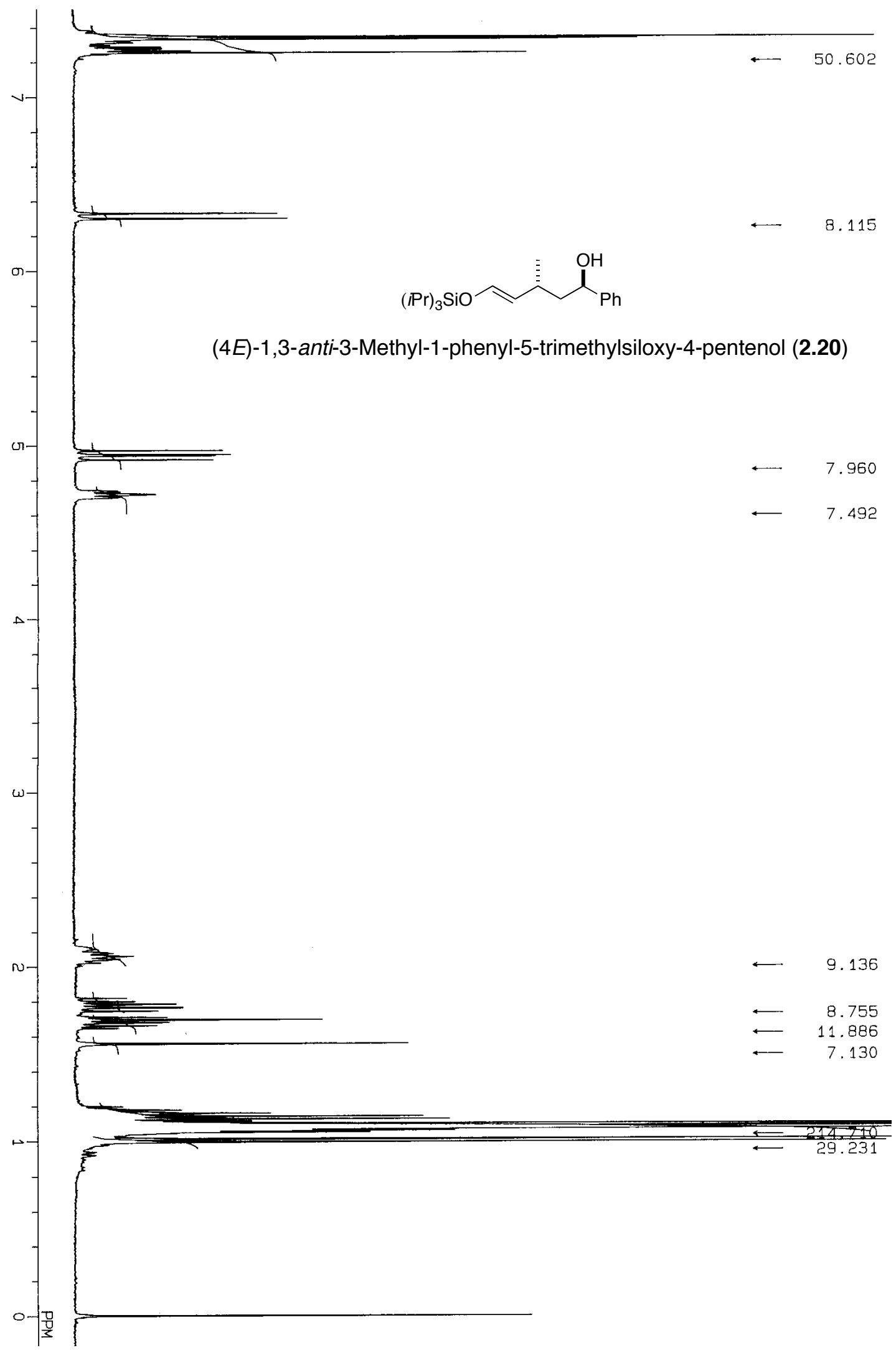




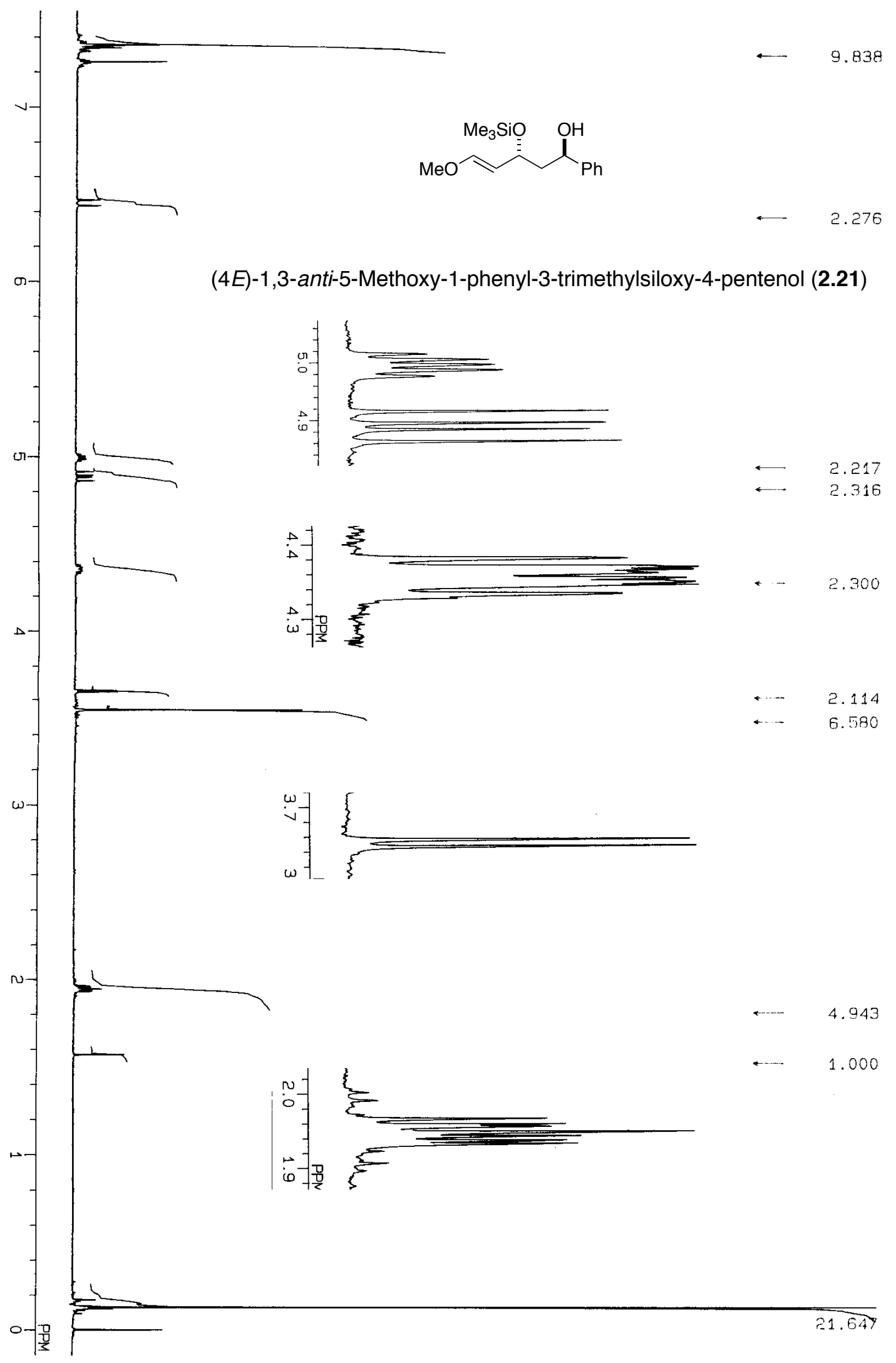




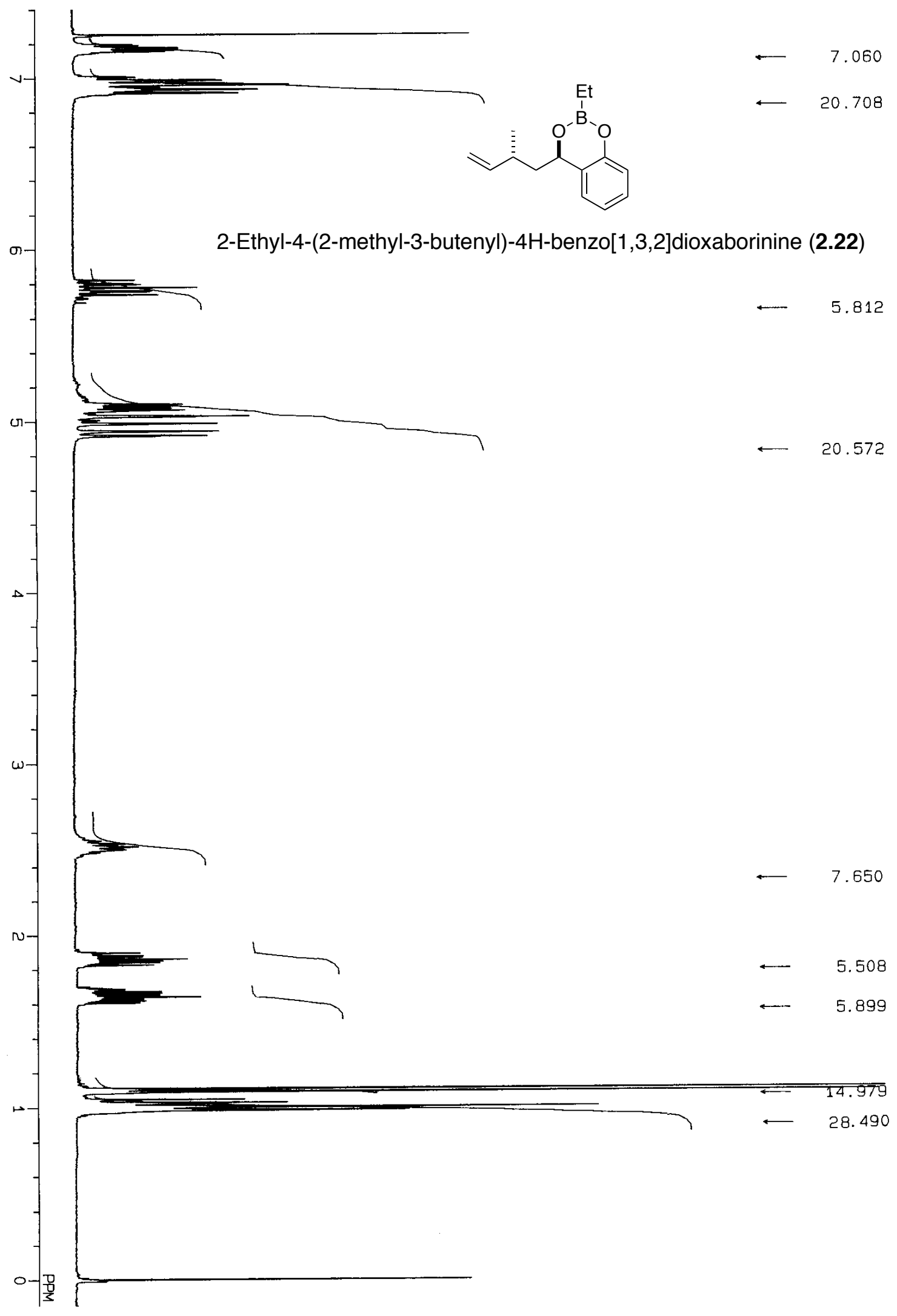

SI-45

45 


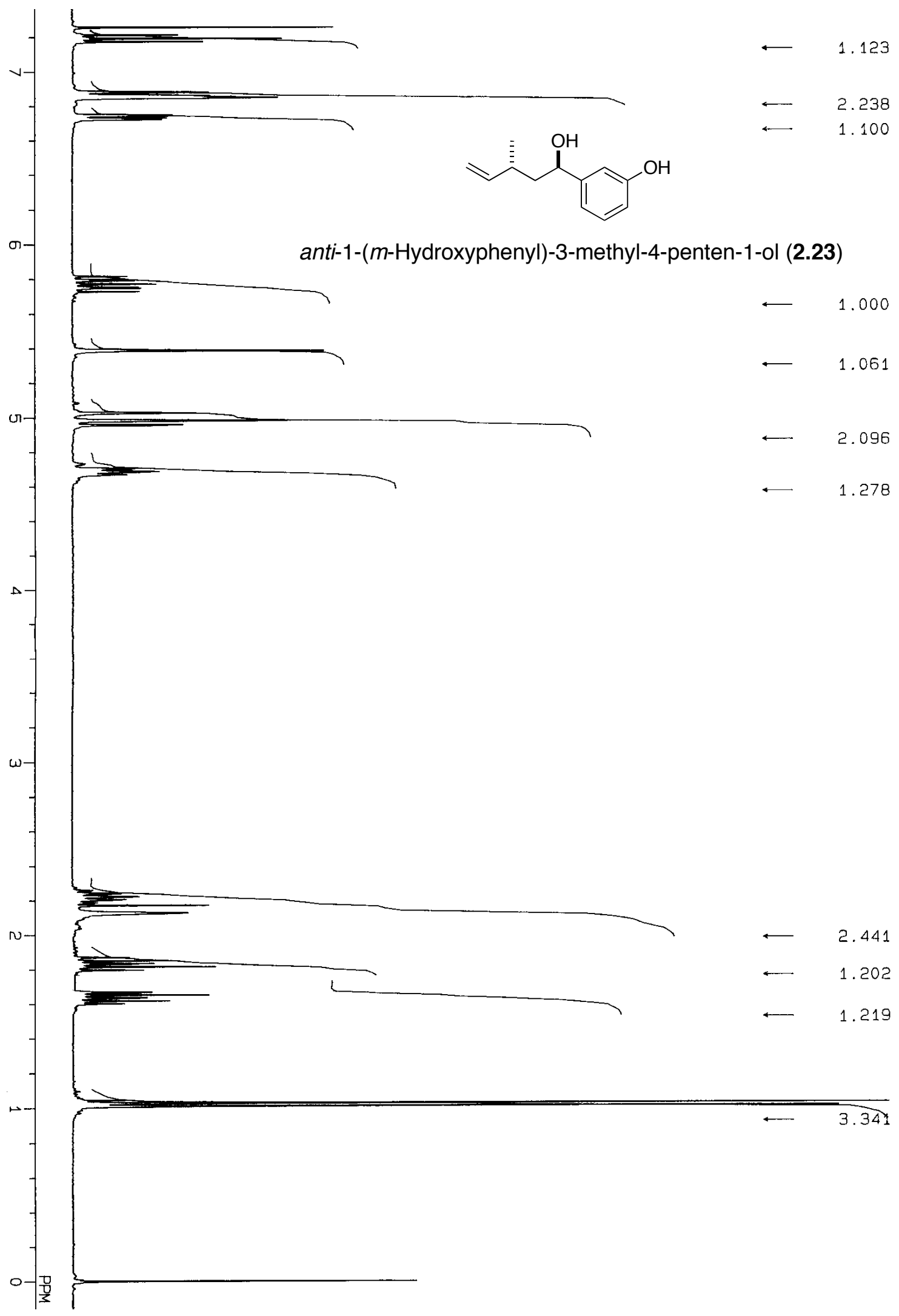




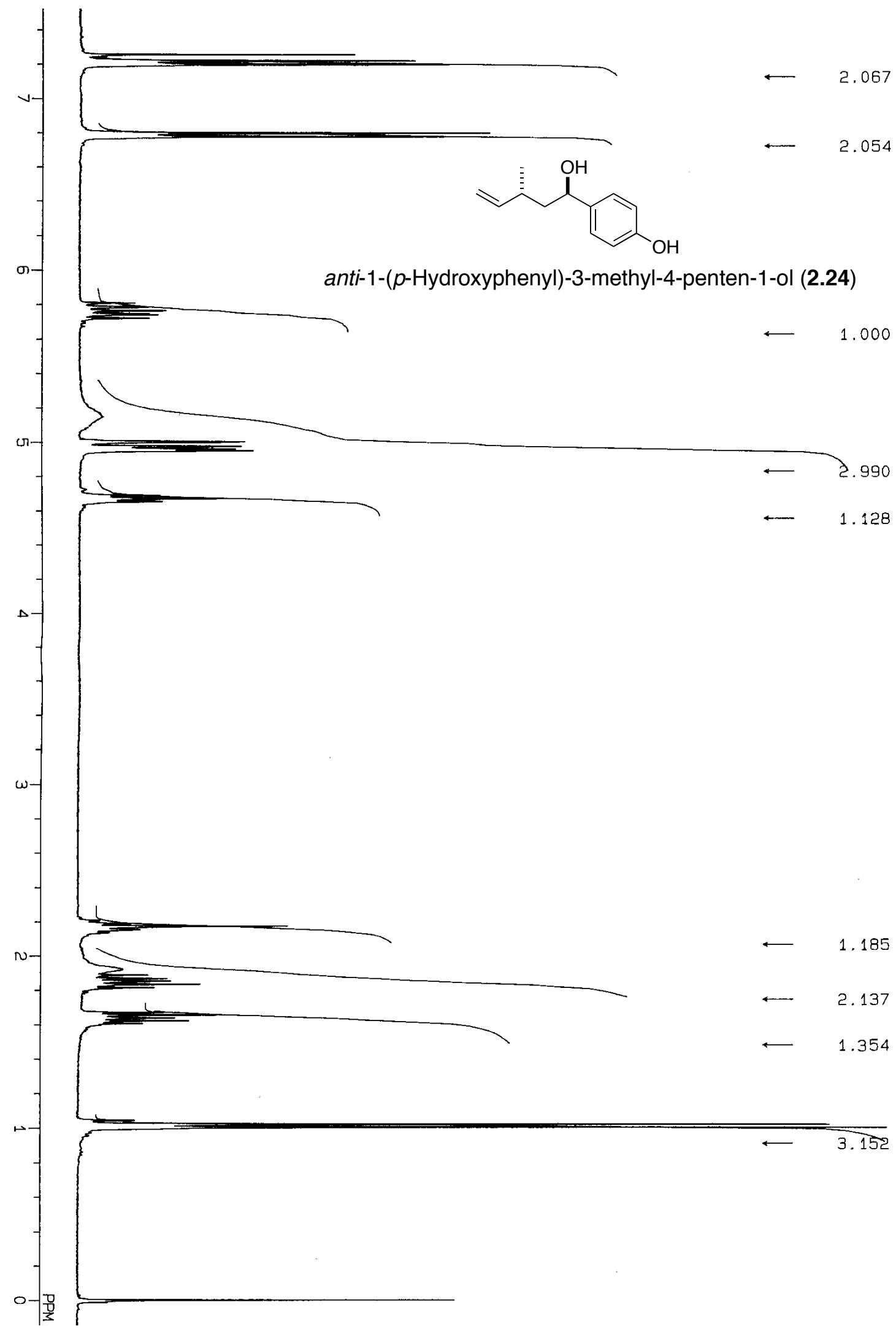




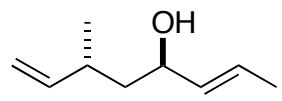

(2E)-4,6-anti-6-Methyl-octa-2,7-dien-4-ol (2.26)

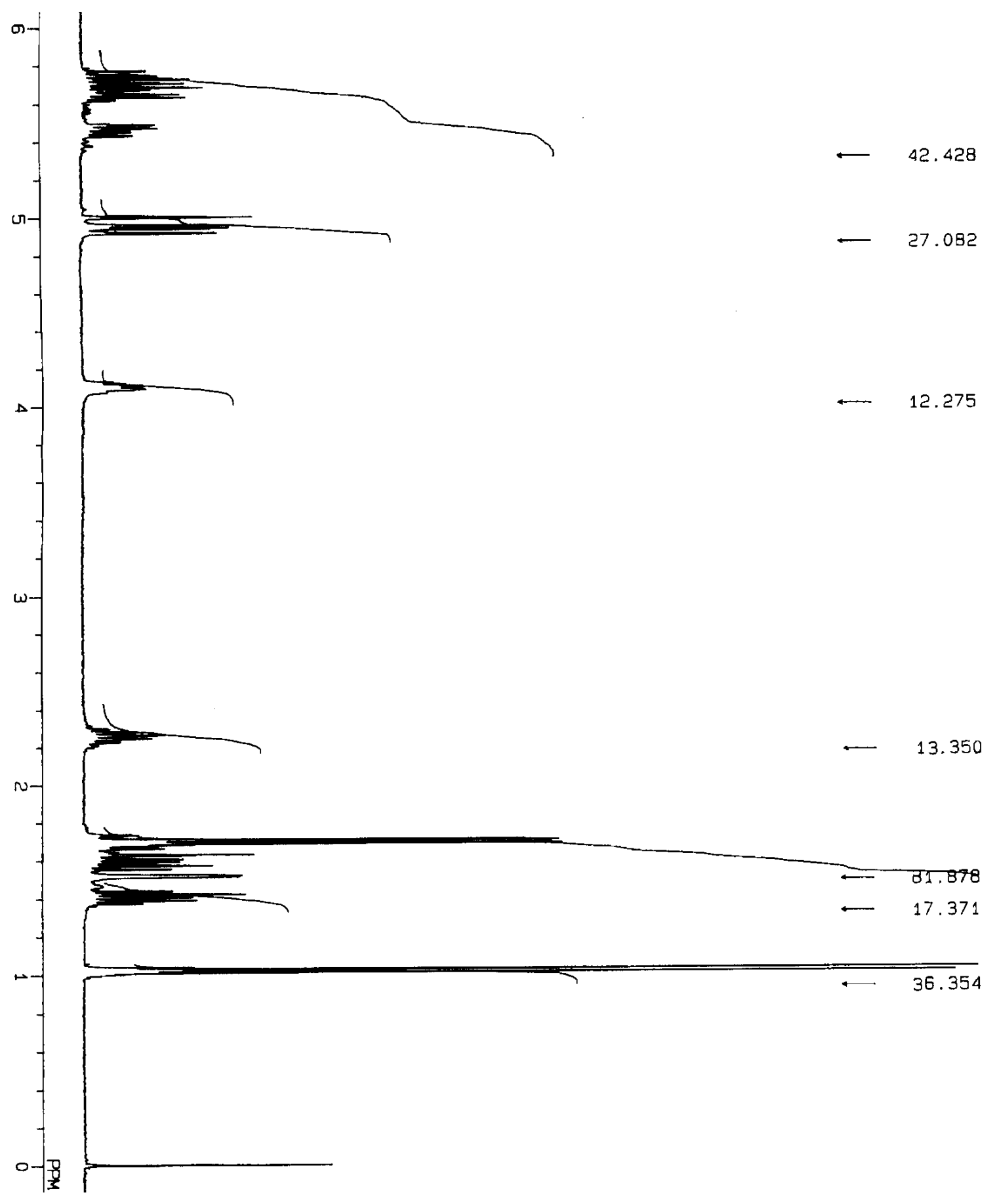




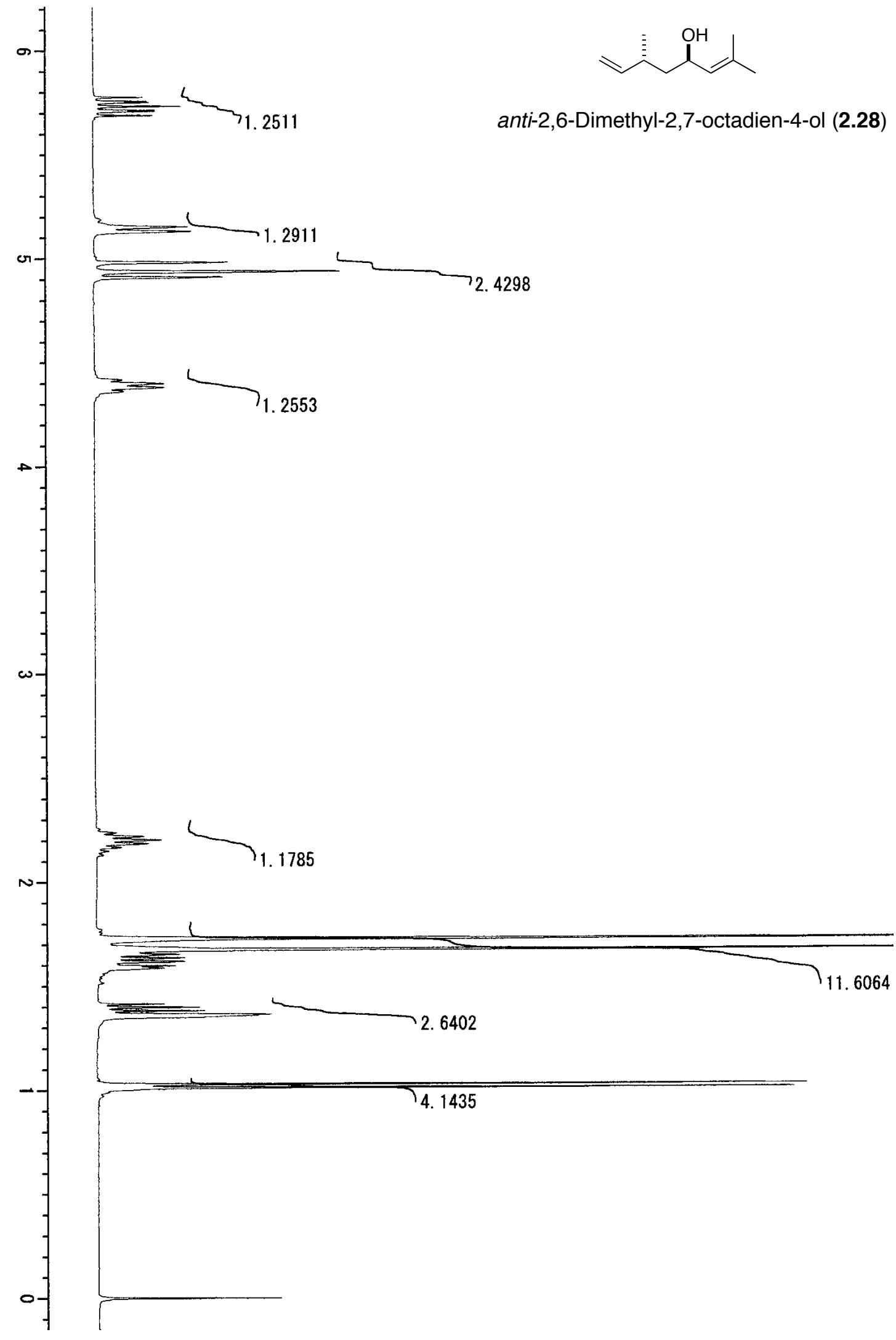




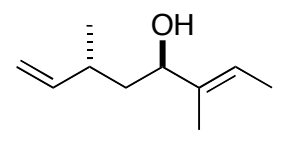

(2E)-4,6-anti-3,6-Dimethyl-2,7-octadien-4-ol (2.29)

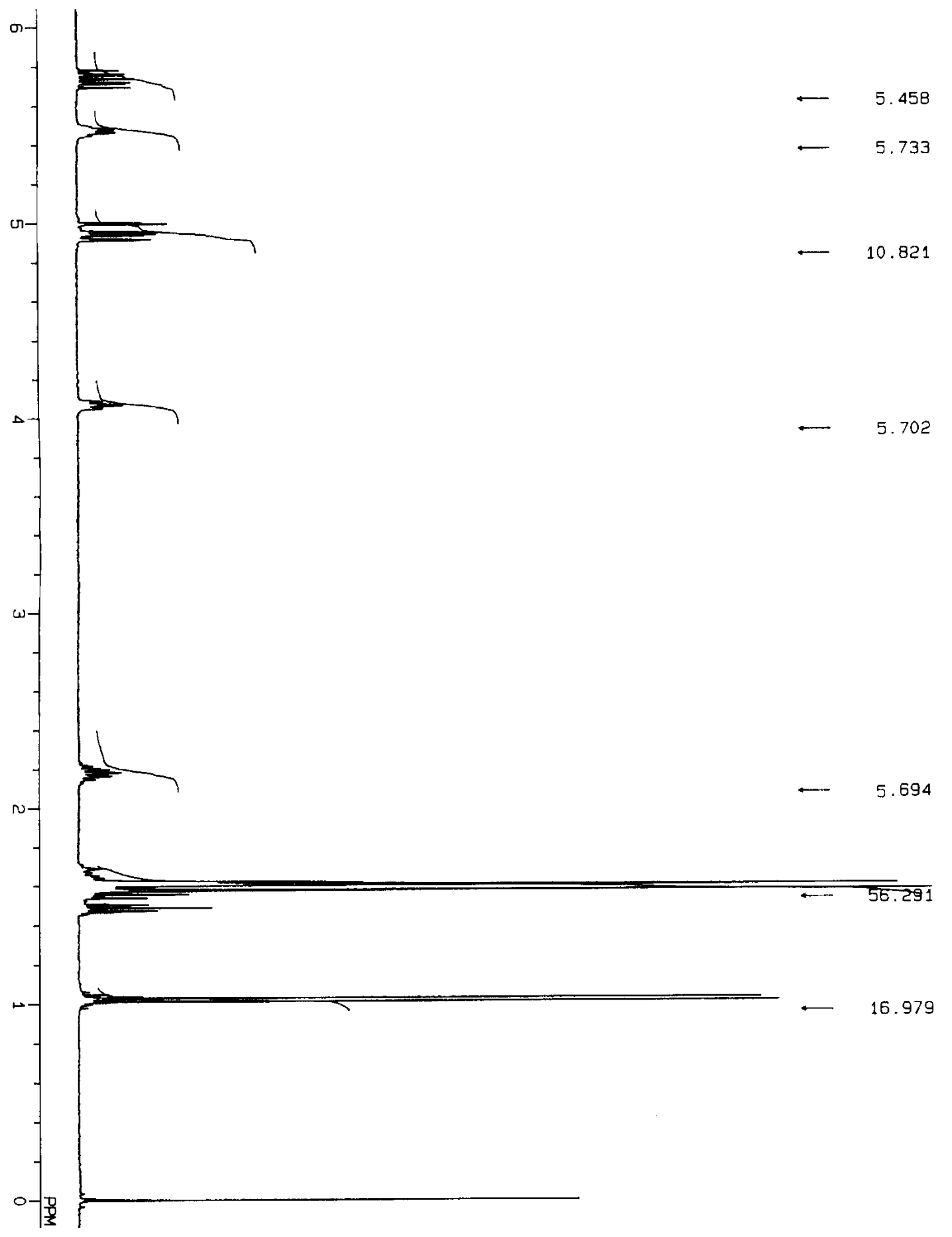




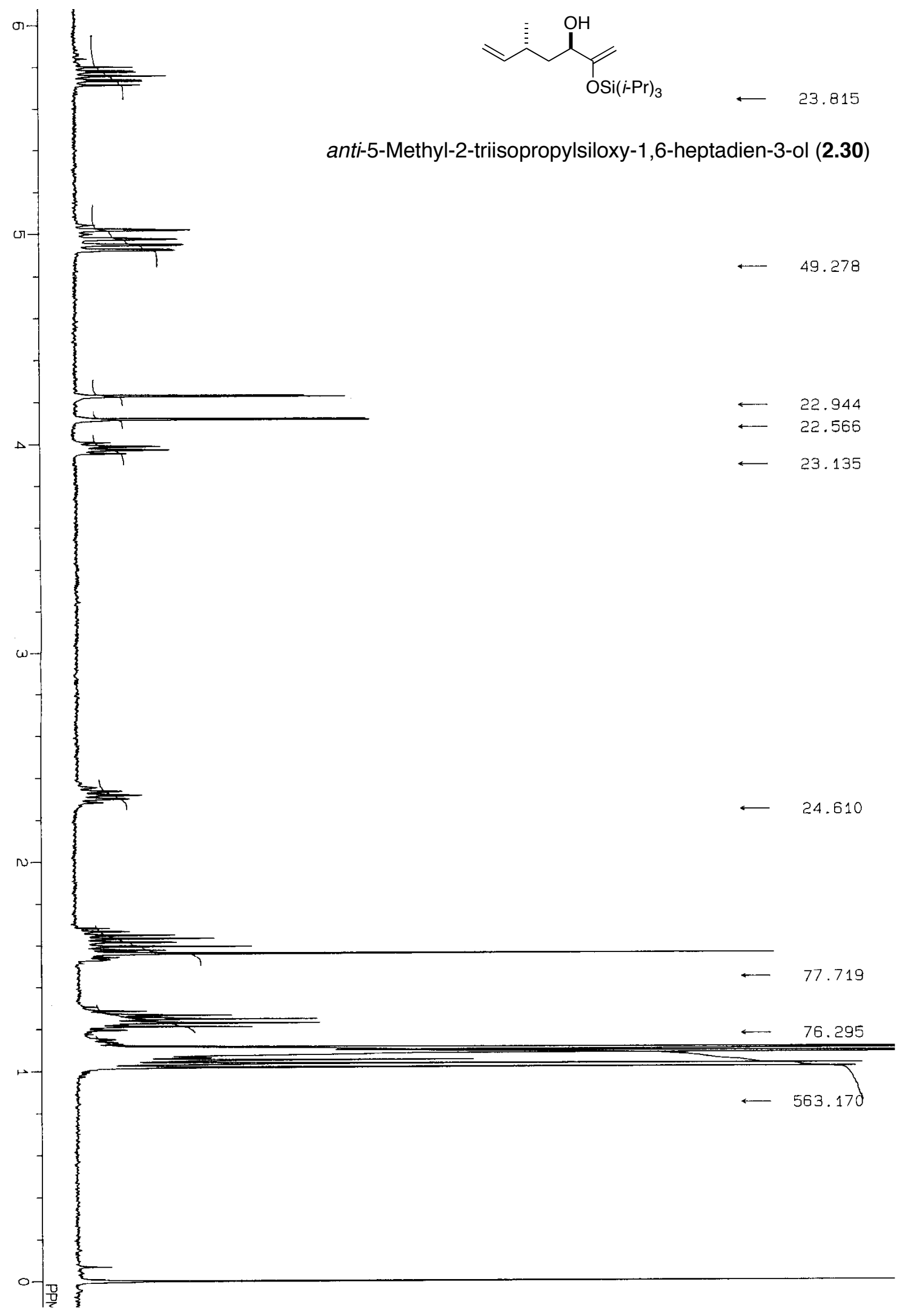




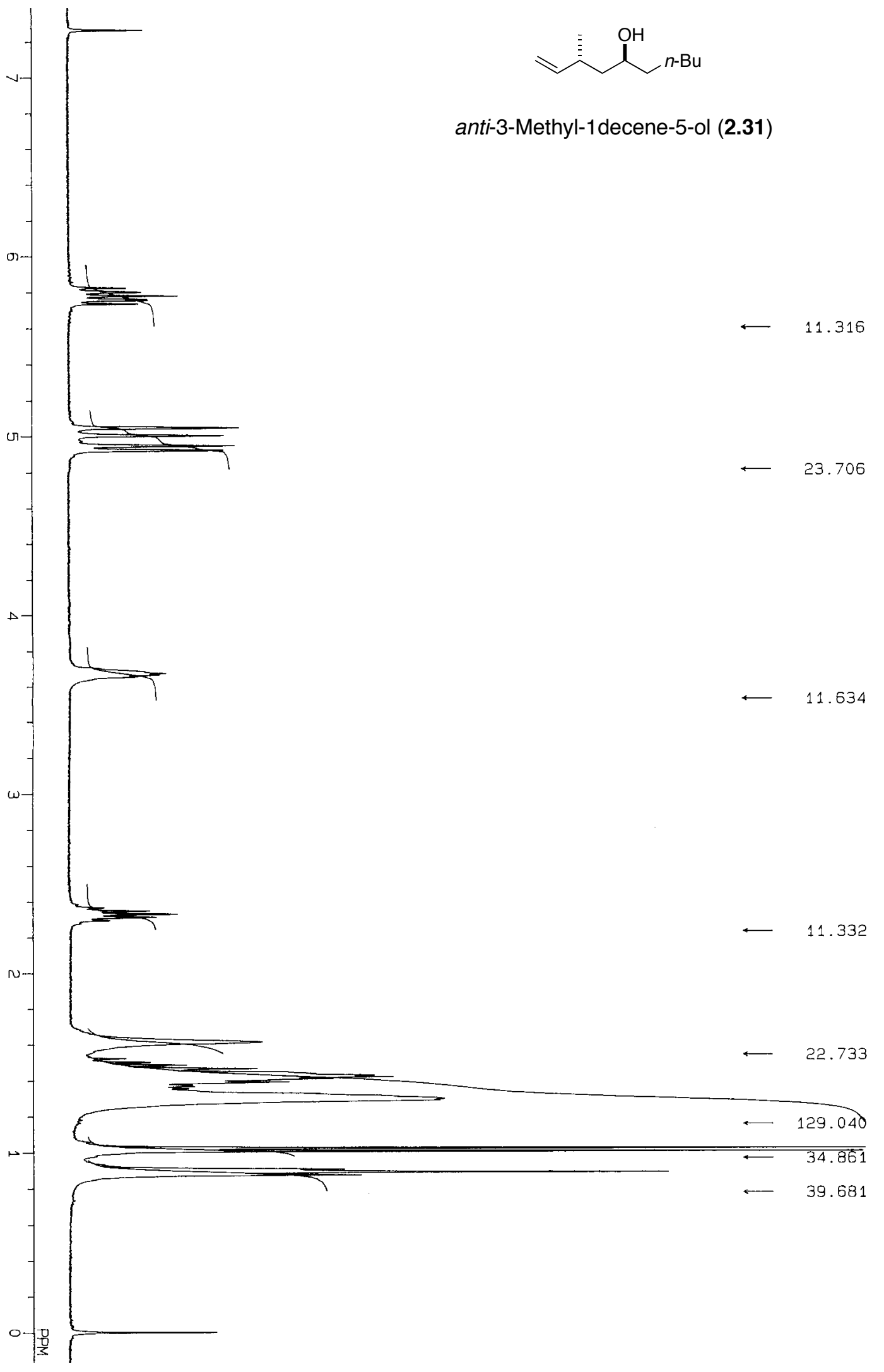




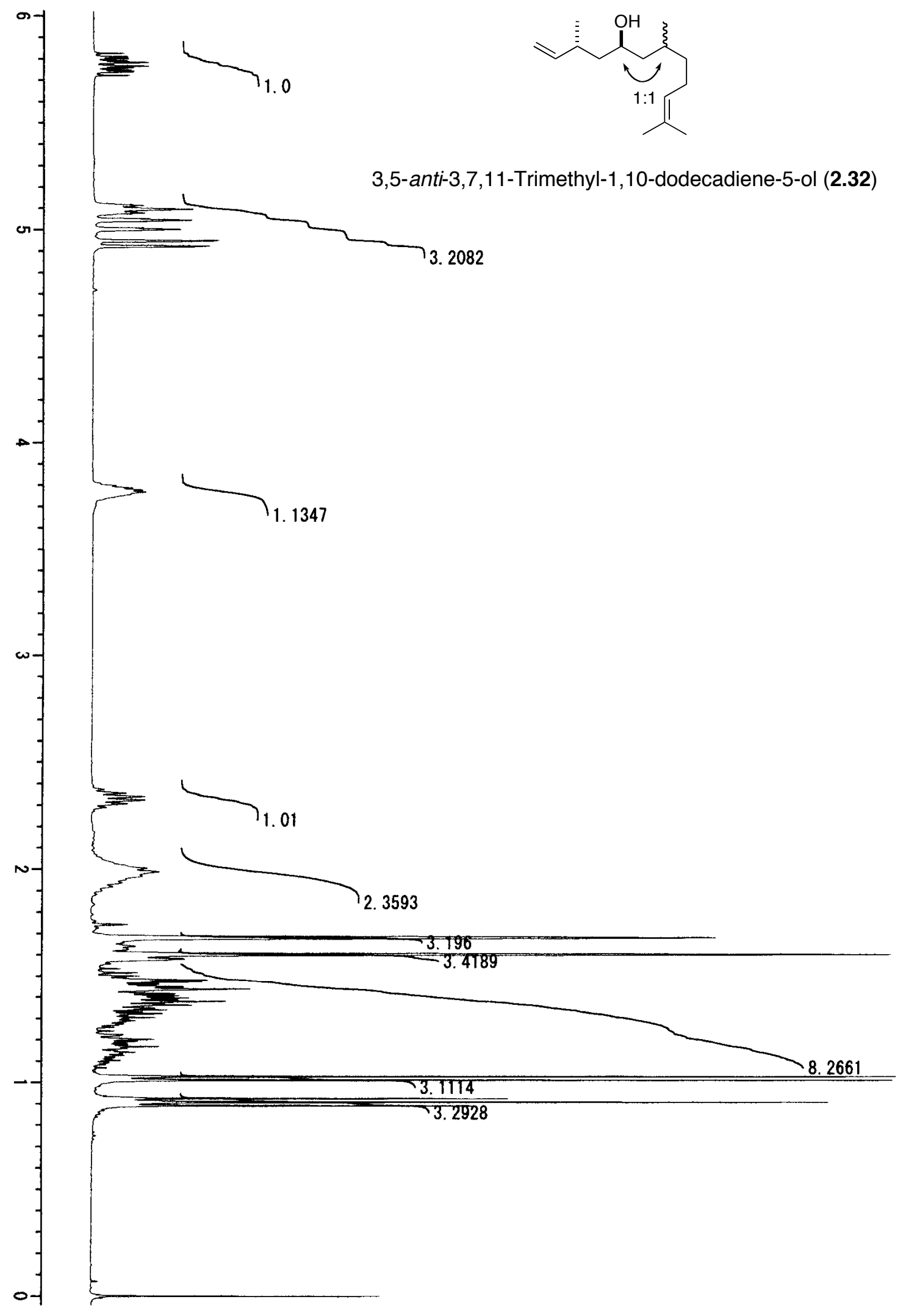




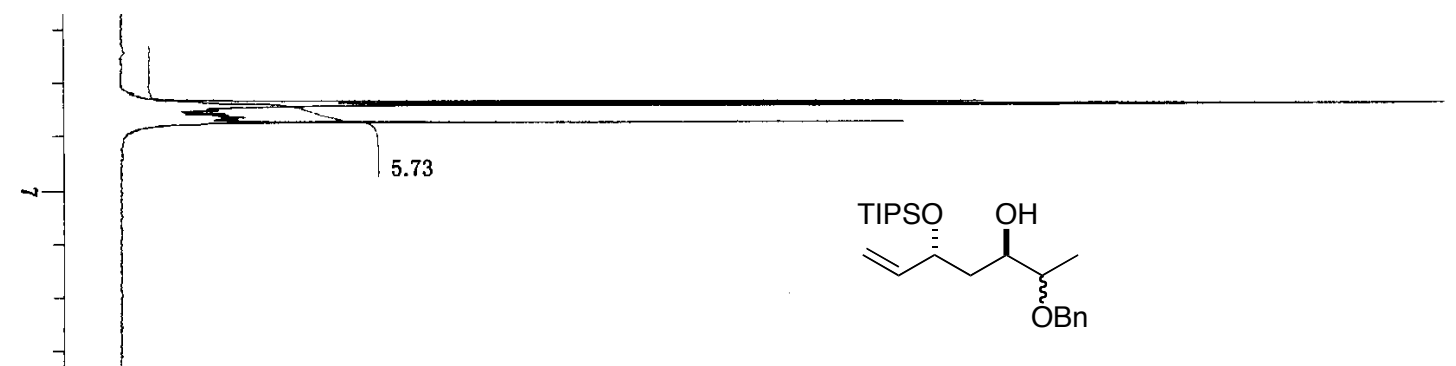

3,5-anti-2-Benzyloxy-5-(triisopropylsiloxy)-6-hepten-3-ol (2.39)

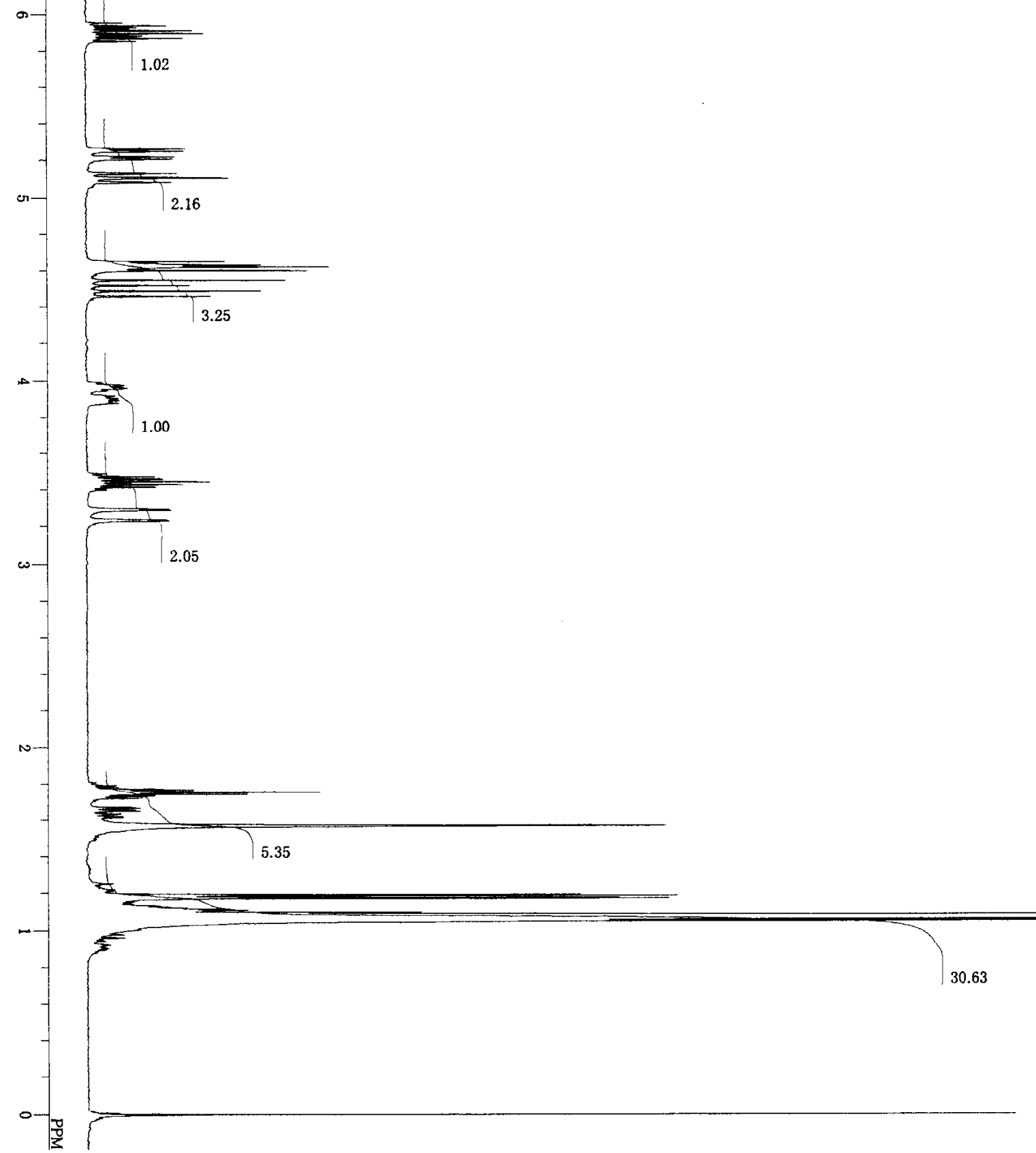



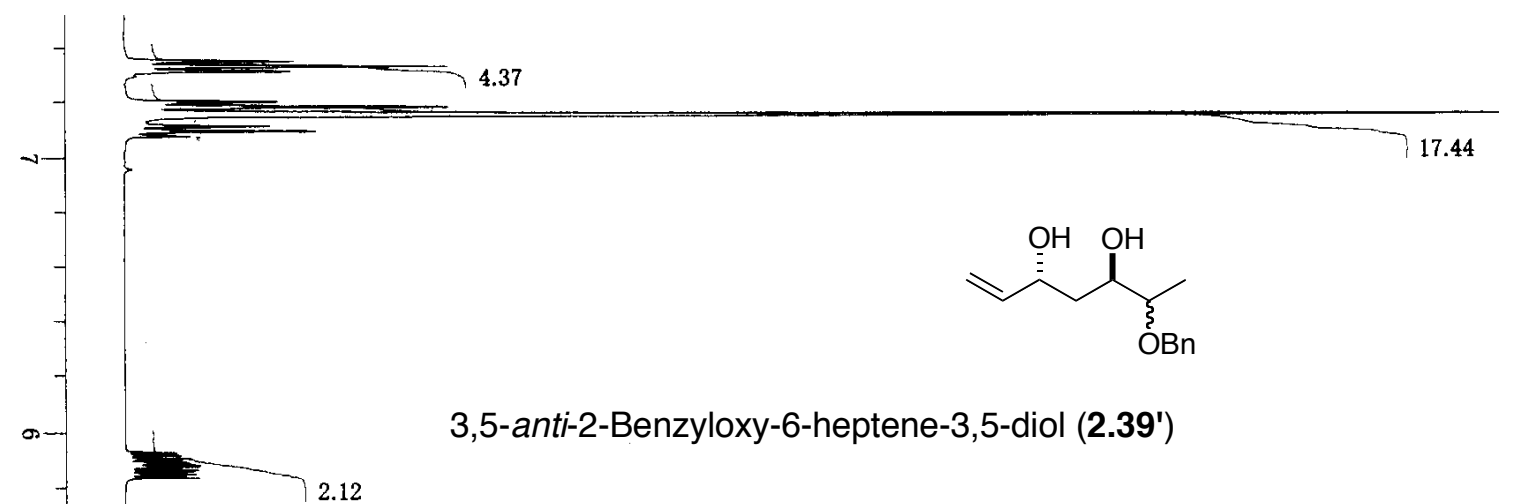

3,5-anti-2-Benzyloxy-6-heptene-3,5-diol (2.39')

$$
\rightarrow
$$

$$
\omega
$$$$
\text { N }
$$

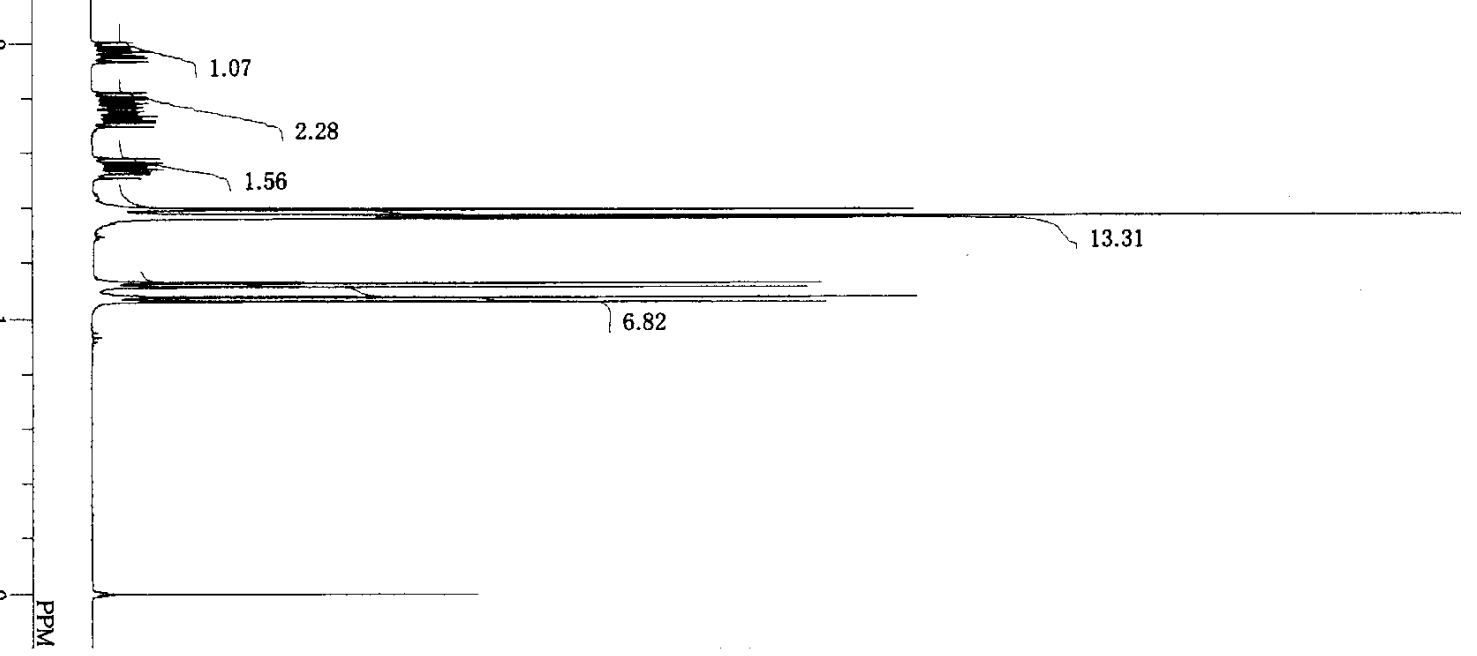




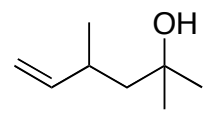

2,4-Dimethyl-5-hexen-2-ol (2.41)

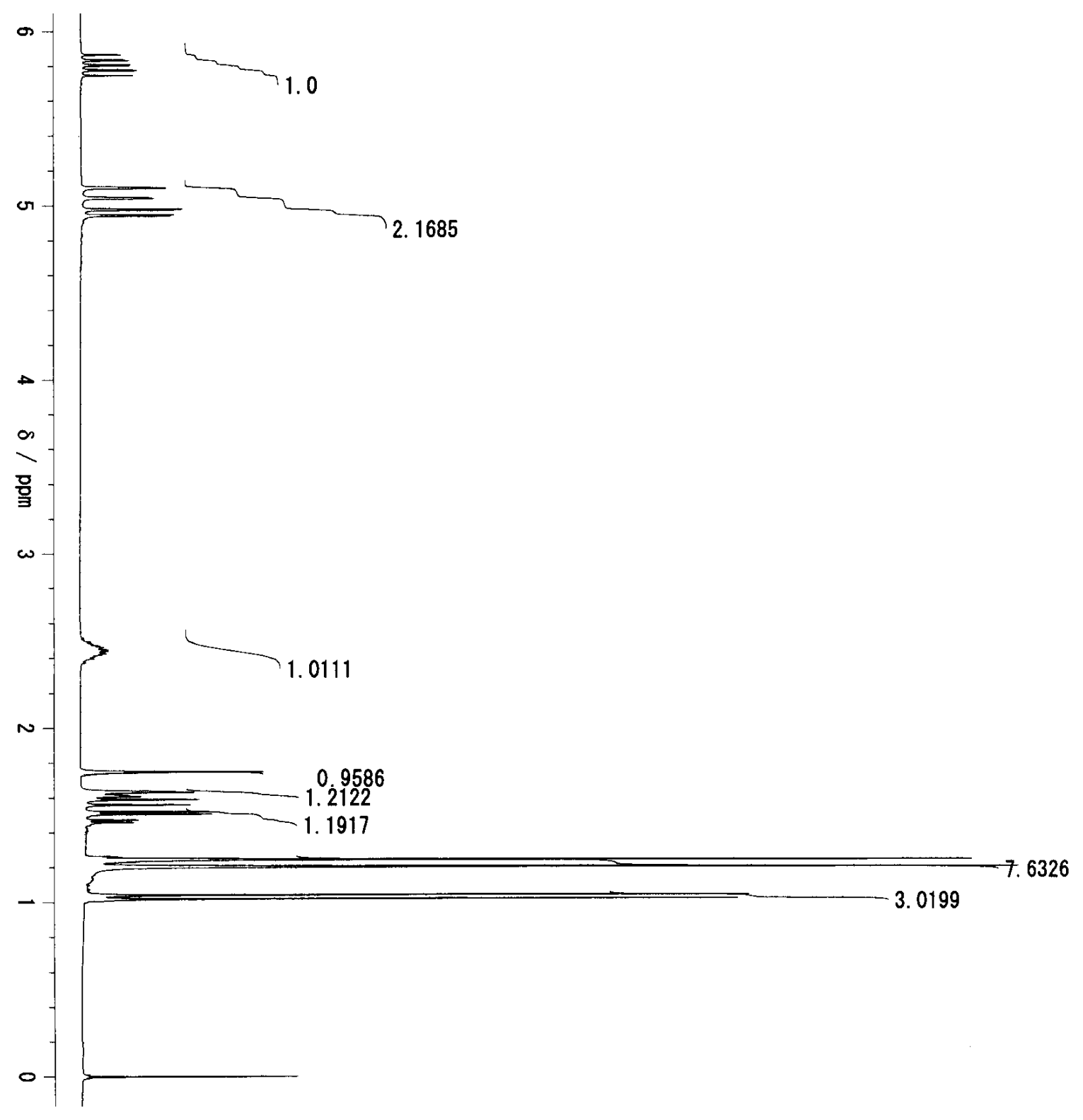




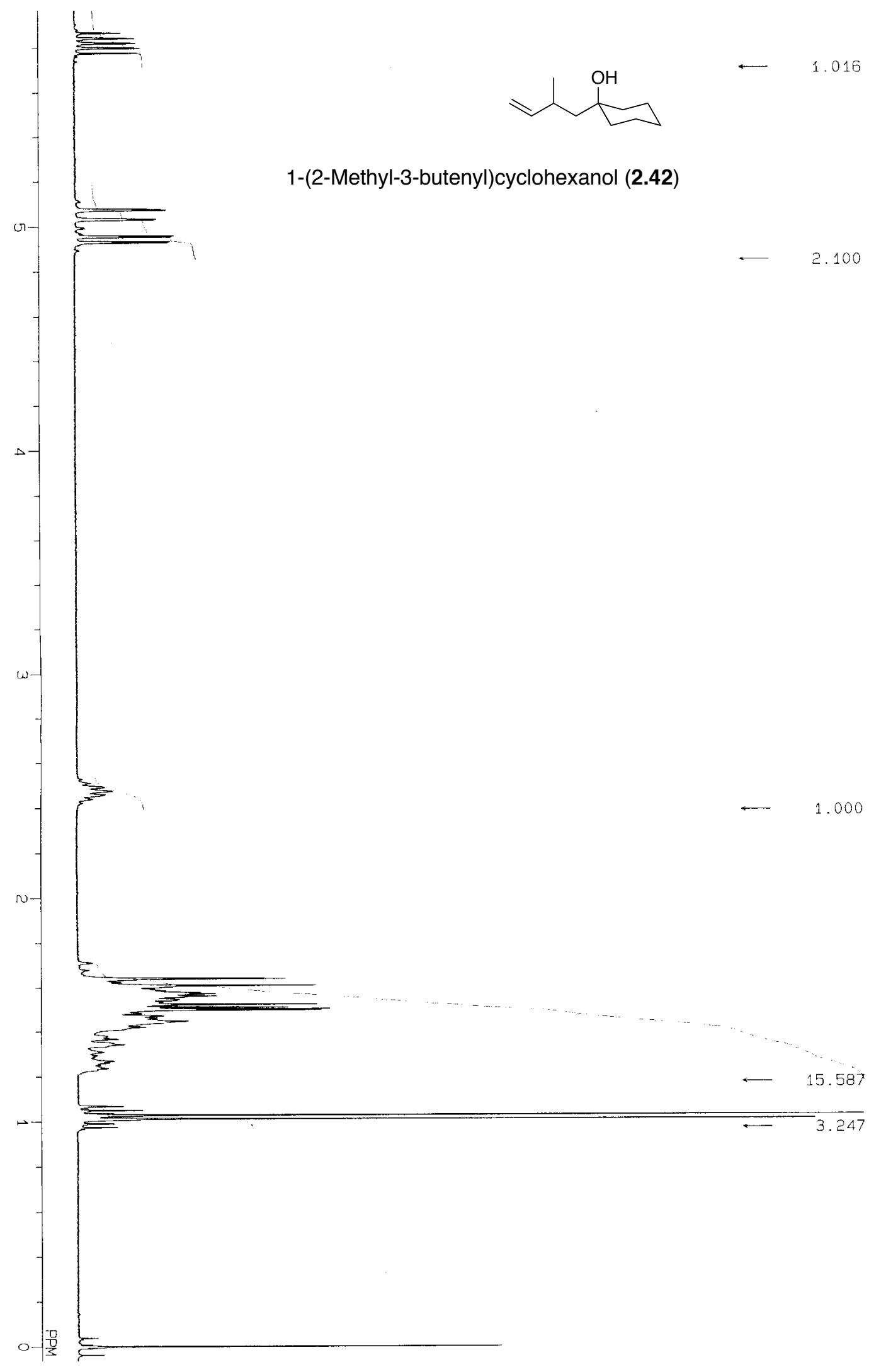




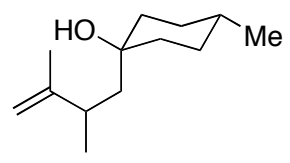

trans-1-(2,3-Dimethyl-3-butenyl)-4-methylcyclohexanol (2.43ax)

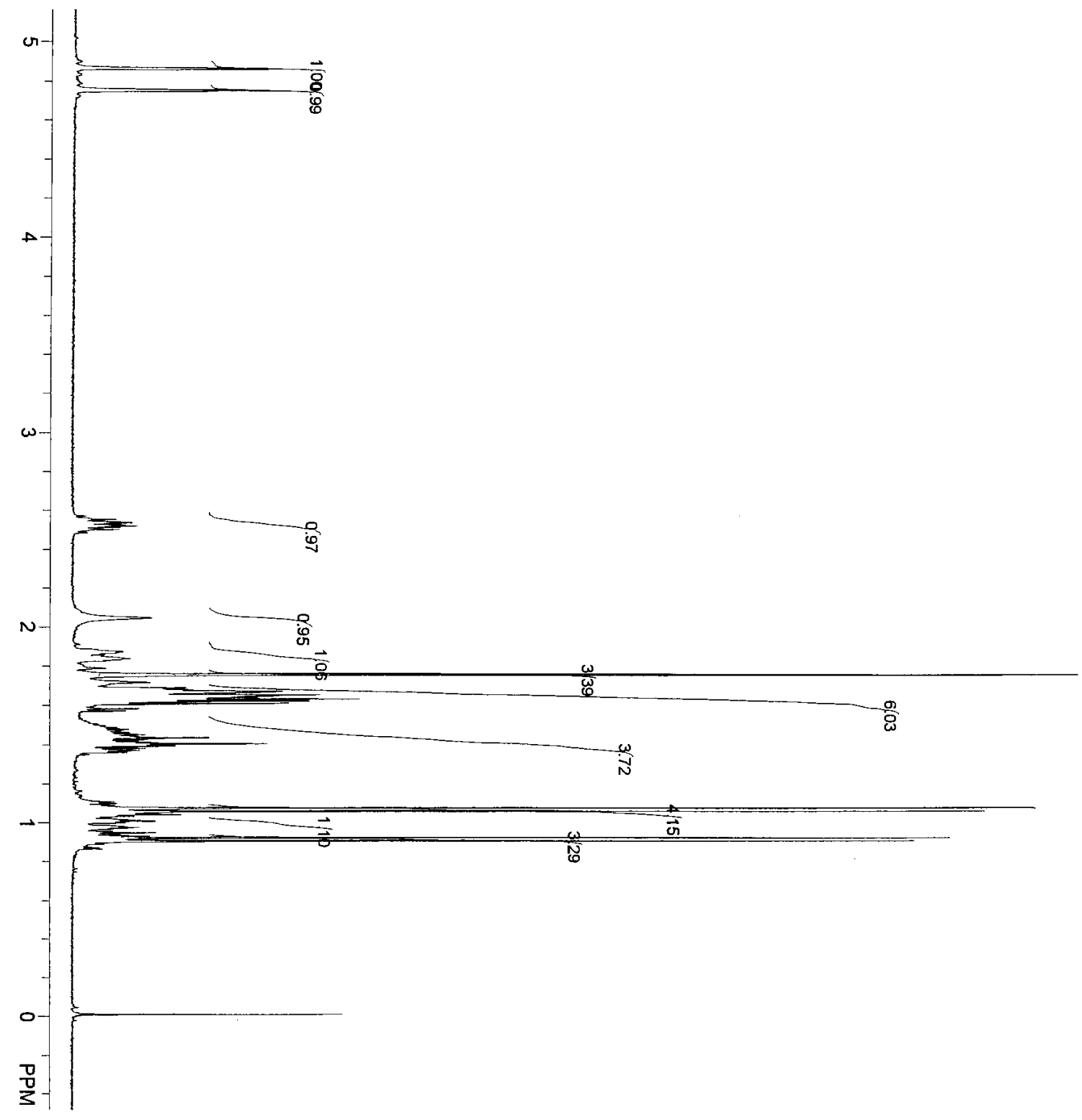




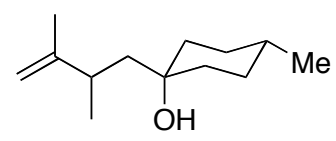

cis-1-(2,3-Dimethyl-3-butenyl)-4-methylcyclohexanol (2.43eq)

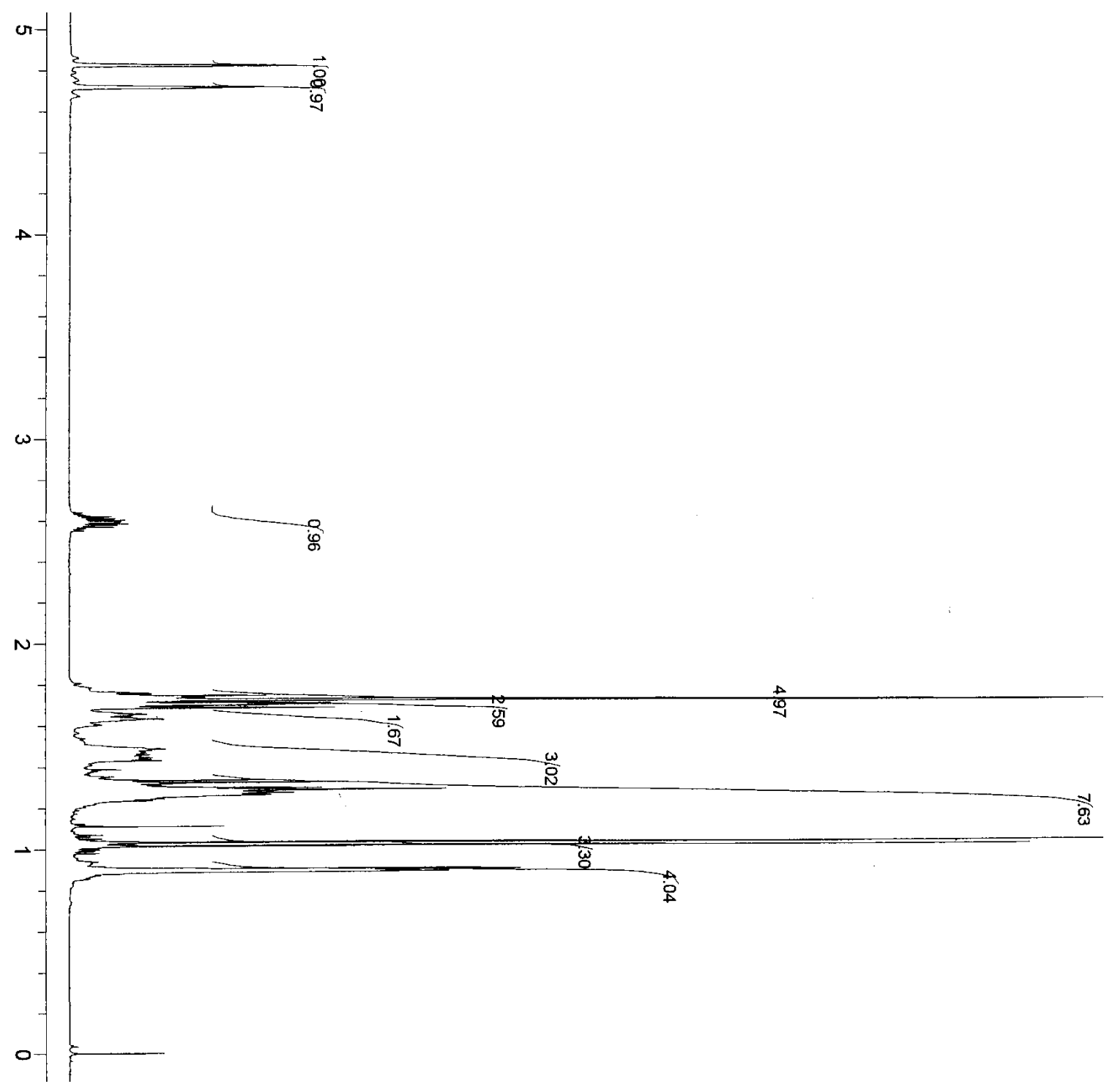




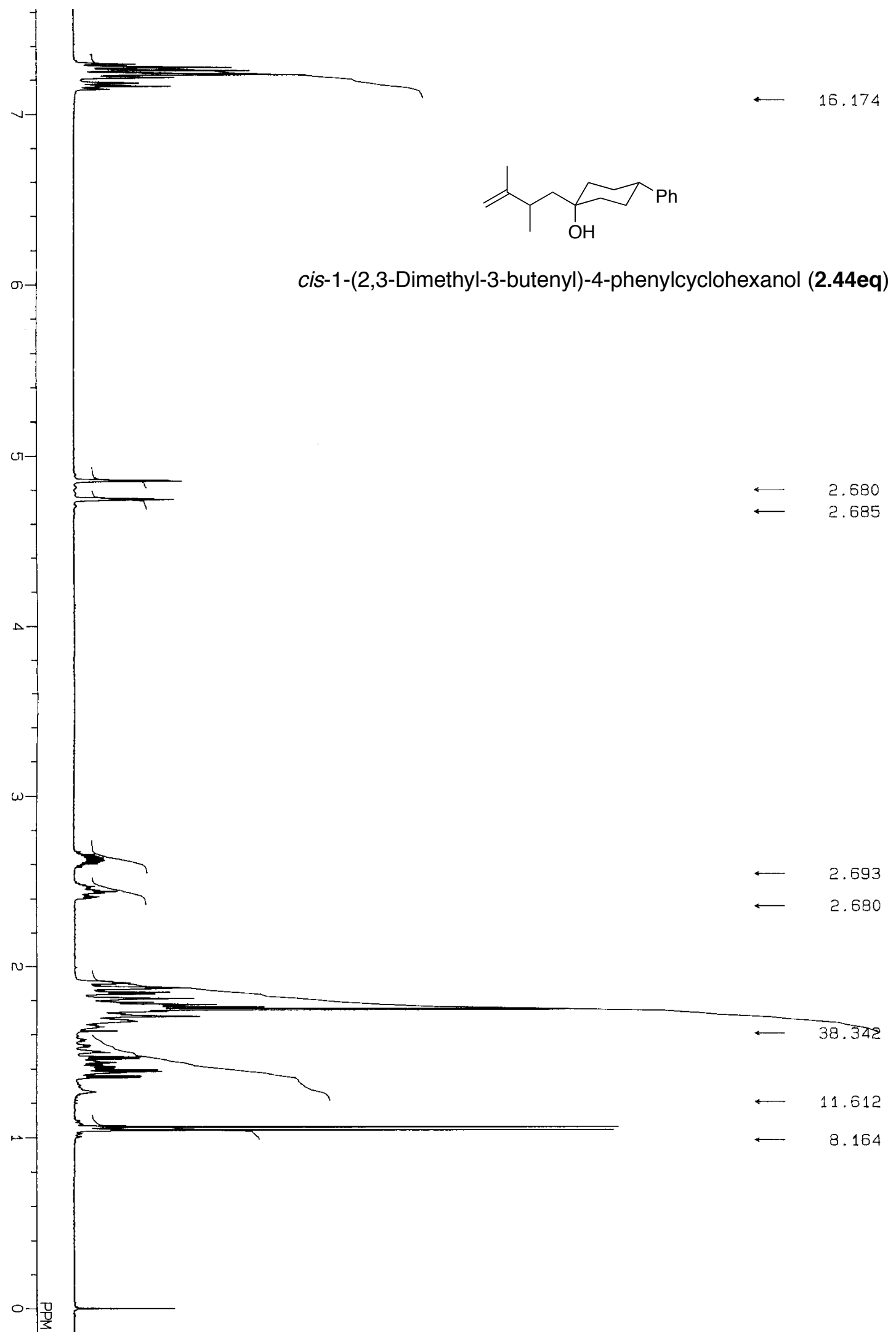




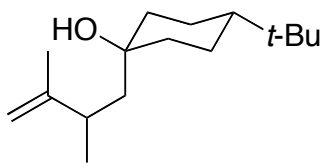

trans-4-t-Butyl-1-(2,3-dimethyl-3-butenyl)cyclohexanol (2.45ax)

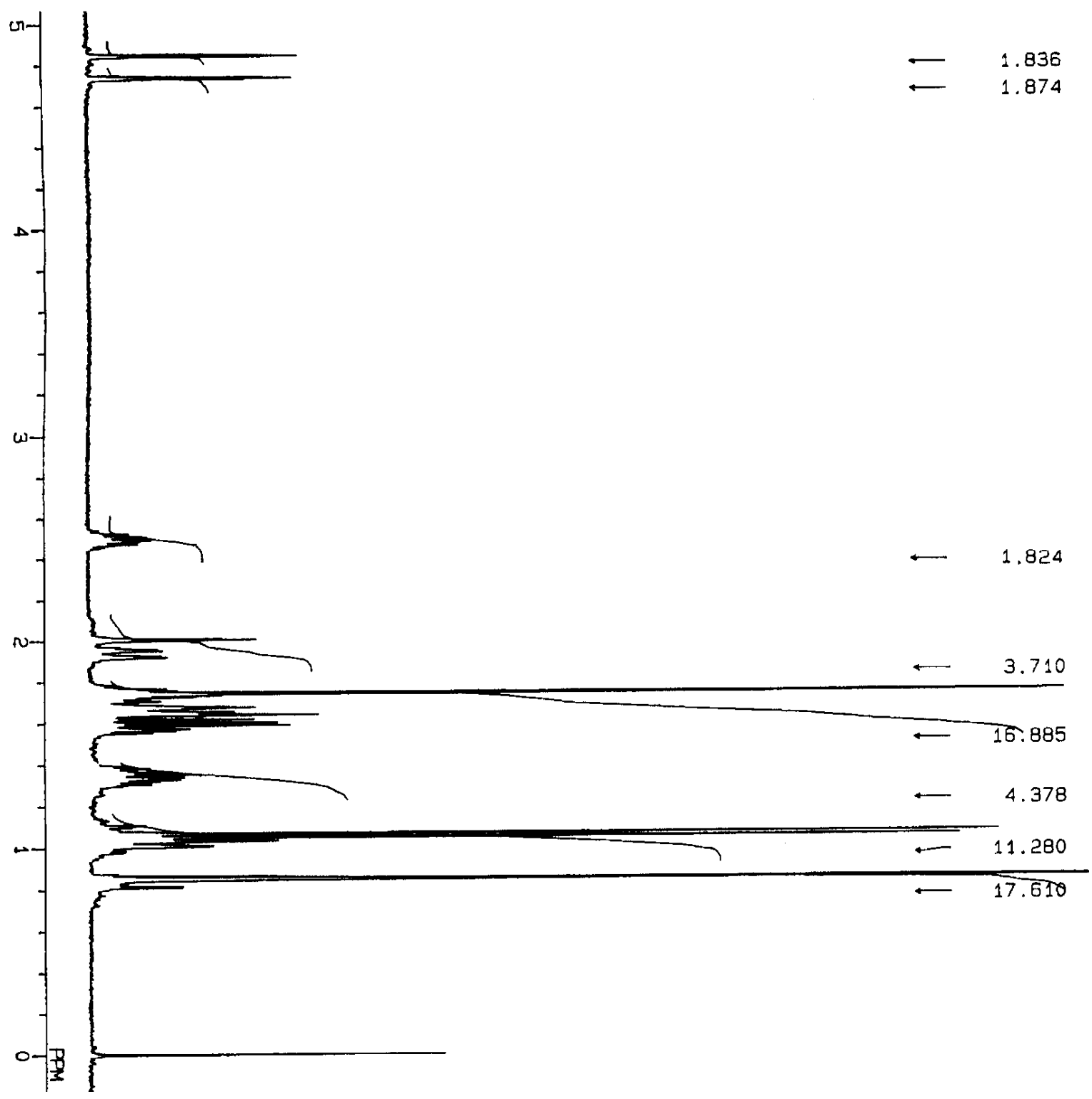




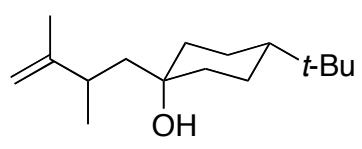

cis-4-t-Butyl-1-(2,3-dimethyl-3-butenyl)cyclohexanol (2.45eq)

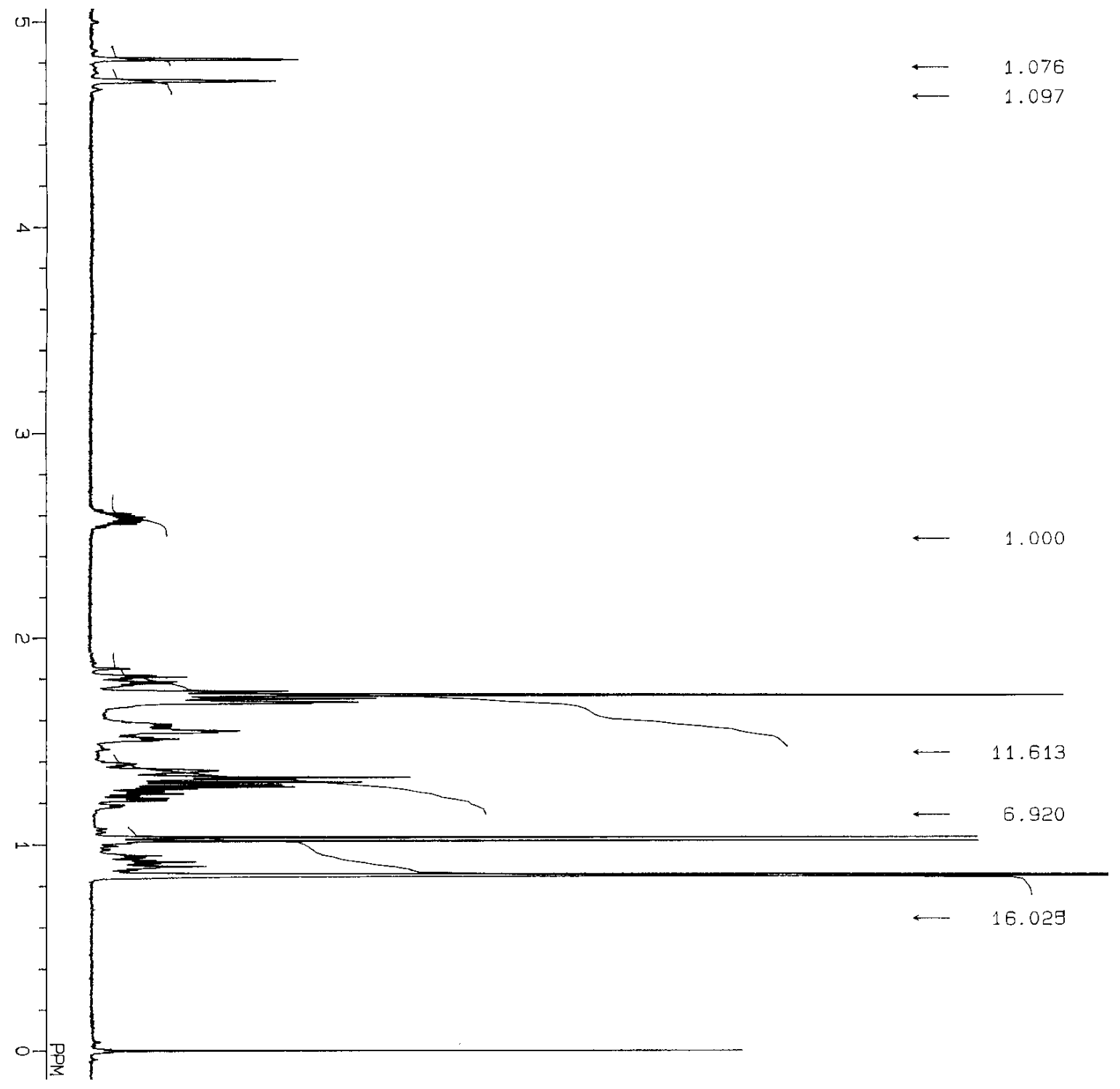



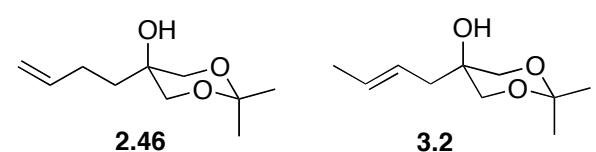

A mixture of $\mathbf{2 . 4 6}$ and $\mathbf{3 . 2}$ in a ratio of 1.3:1

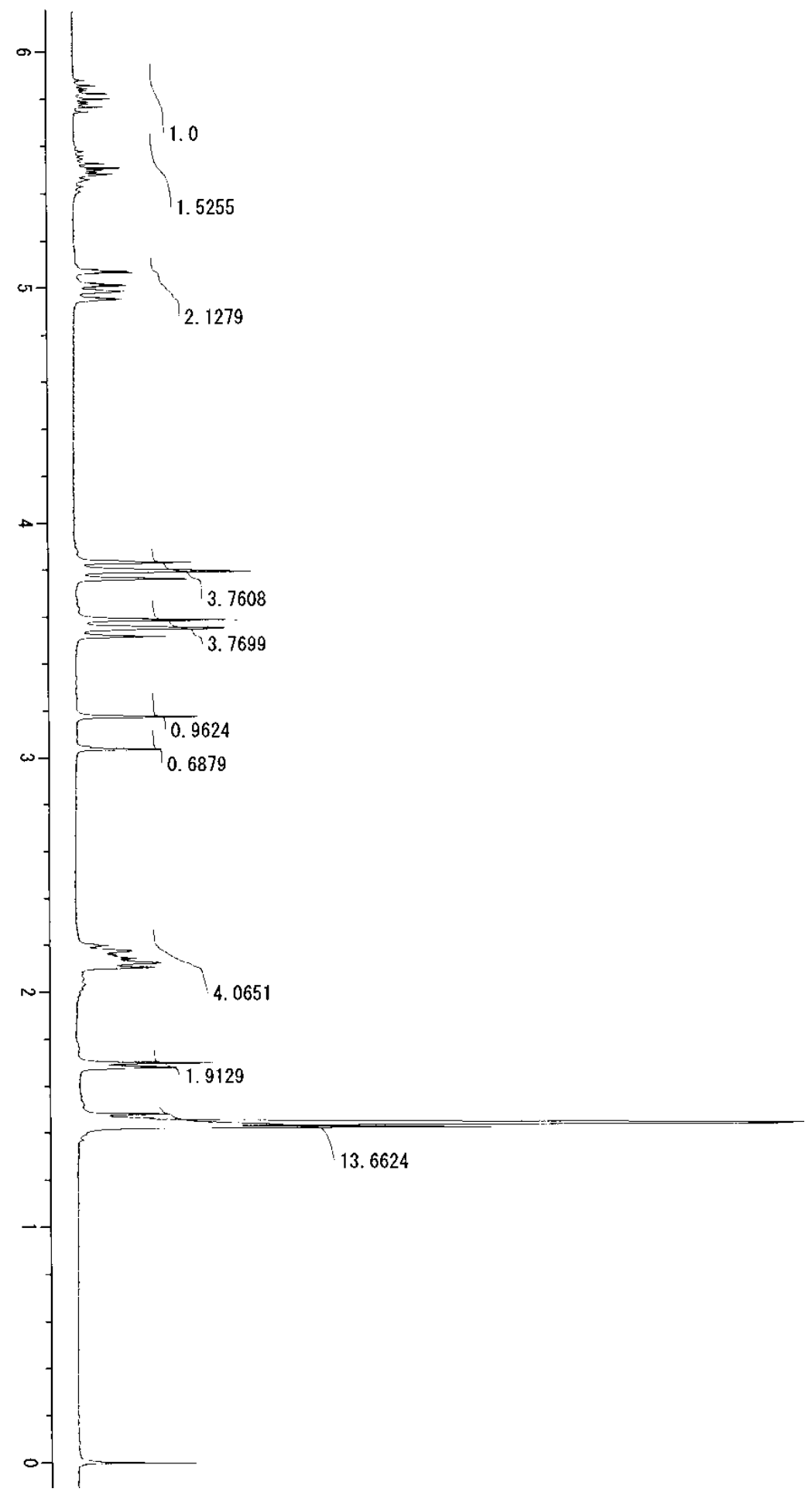



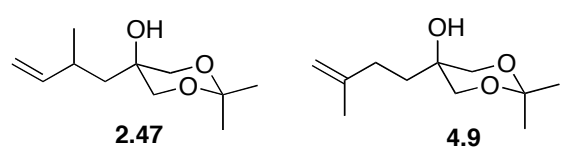

A mixture of 2.47 and 4.9 in a ratio of $4.7: 1$

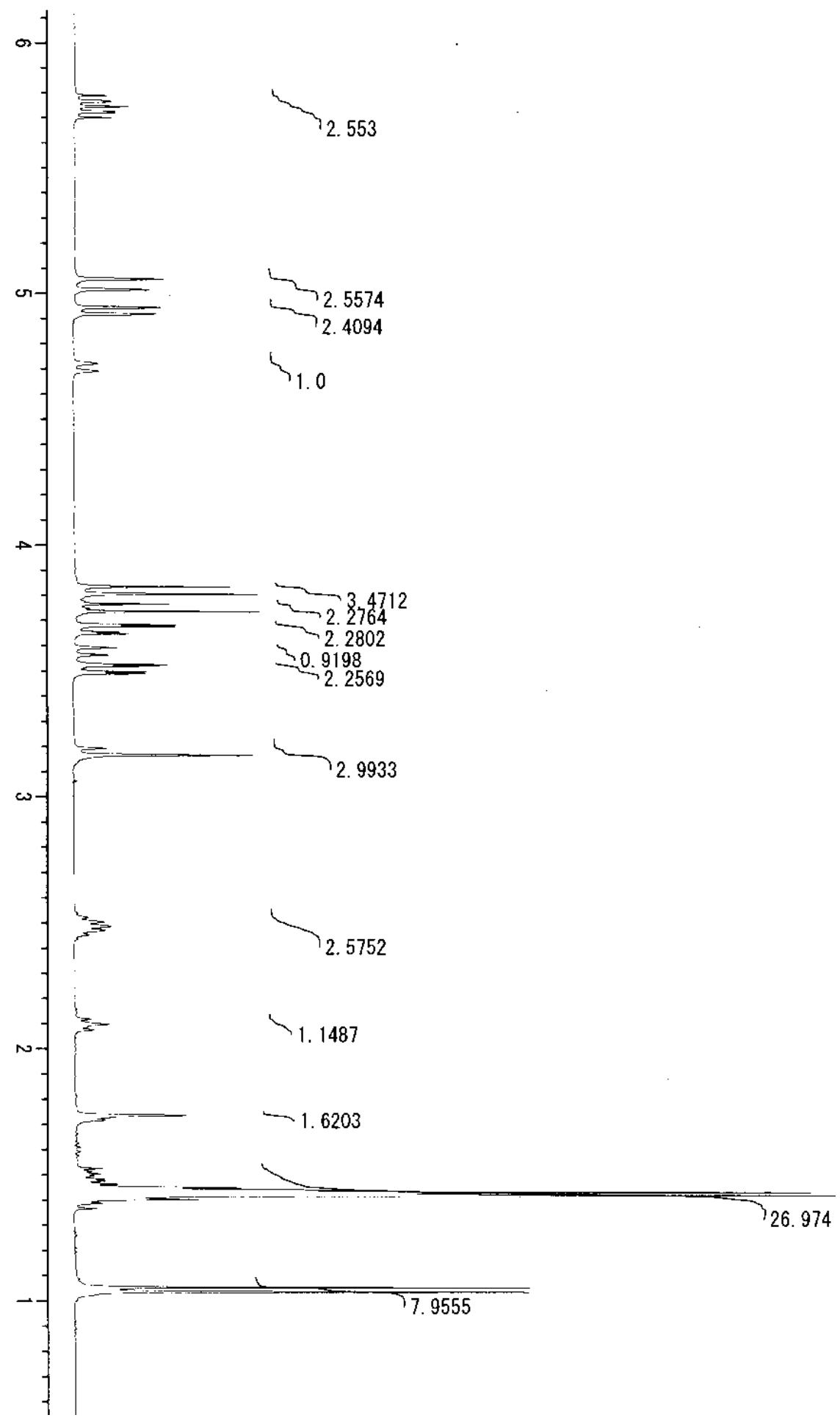




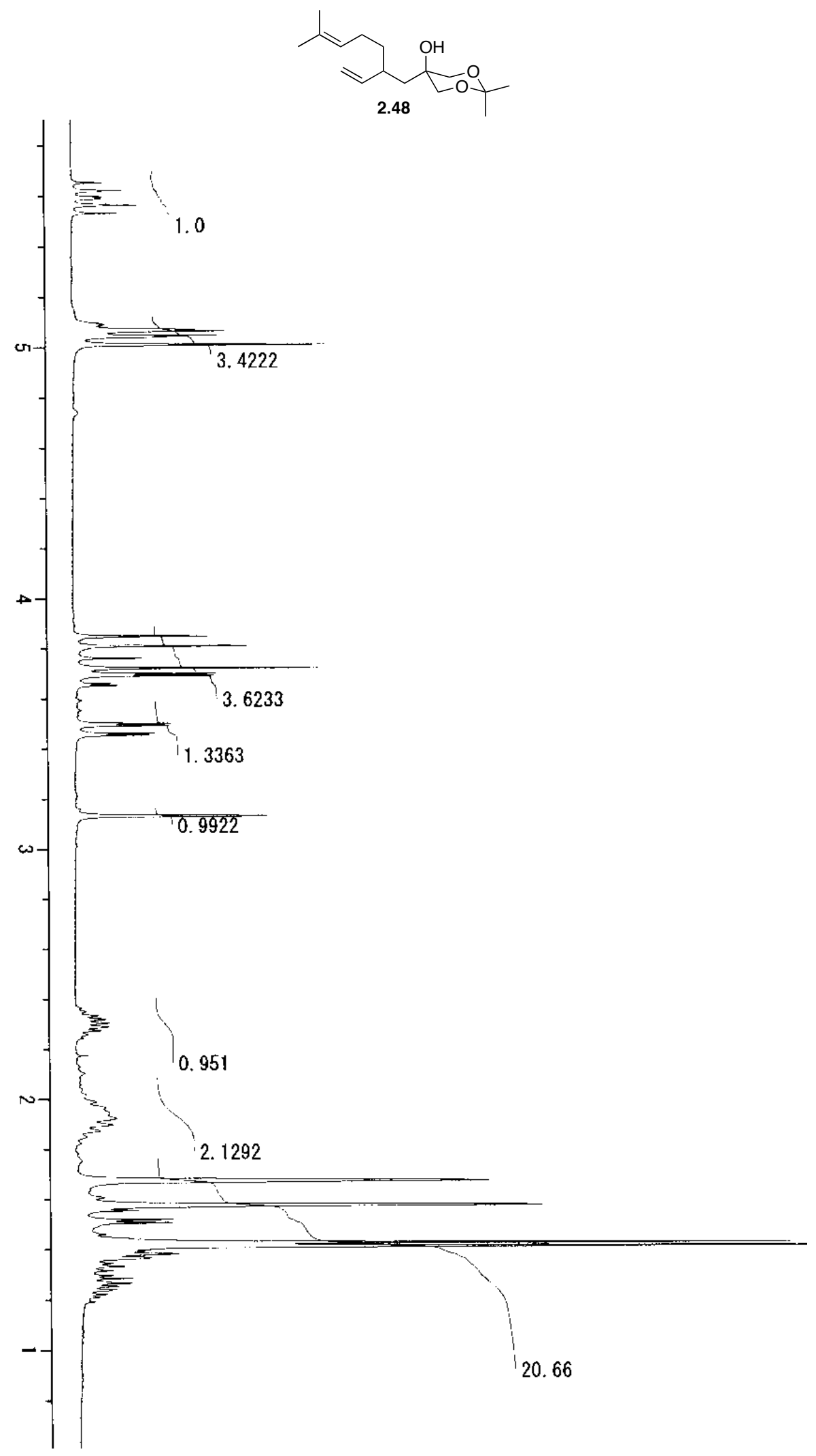




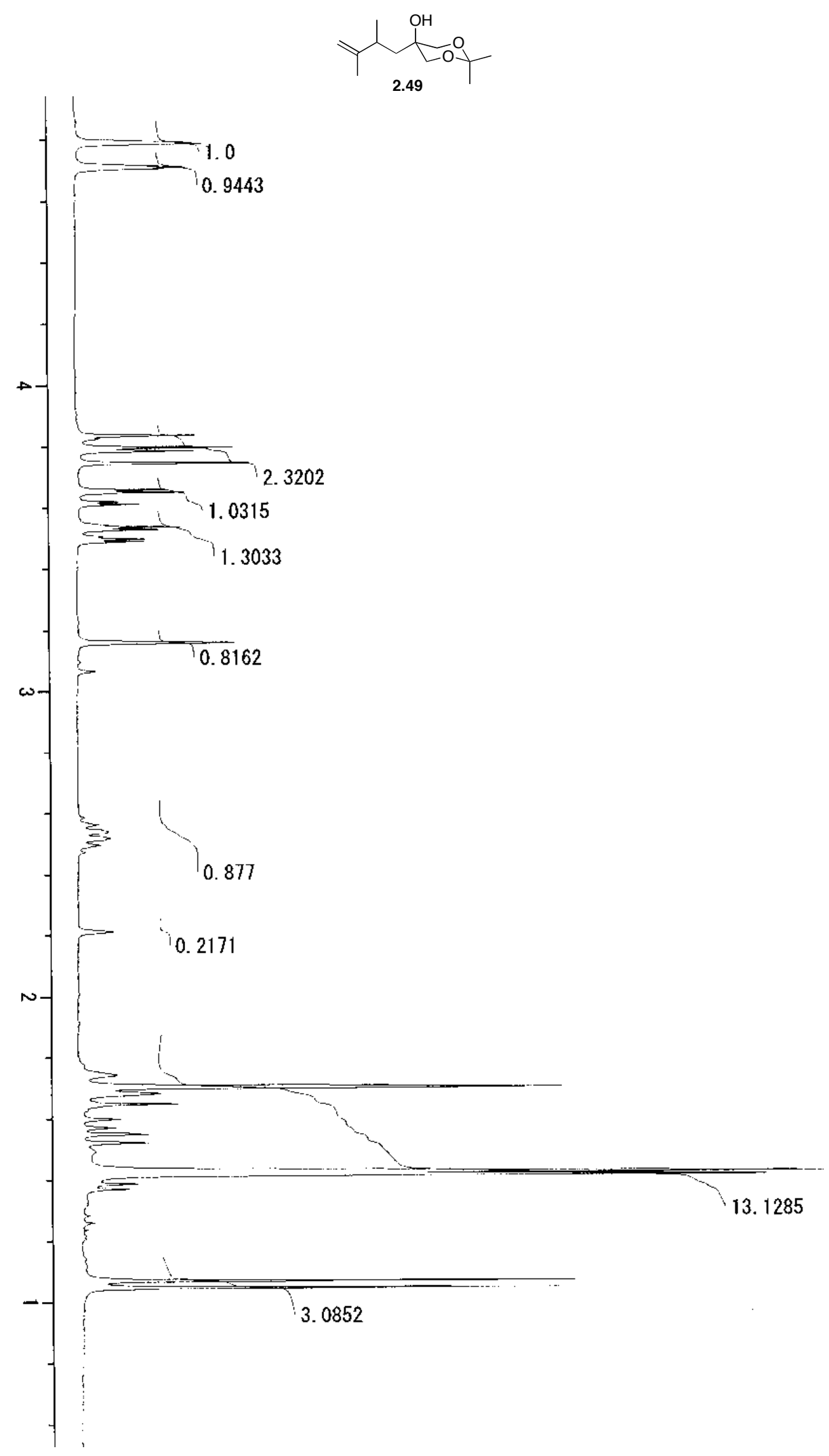




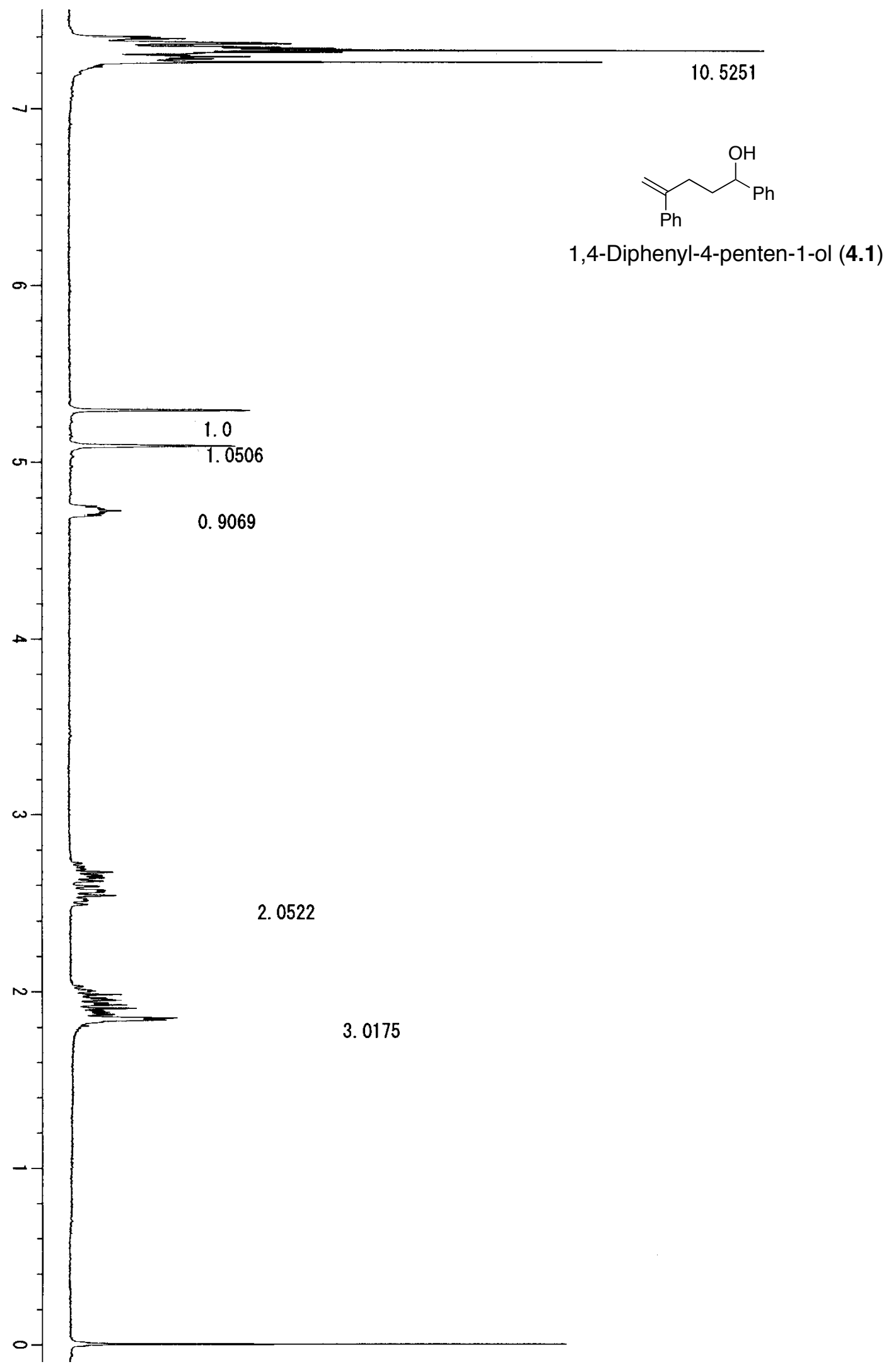




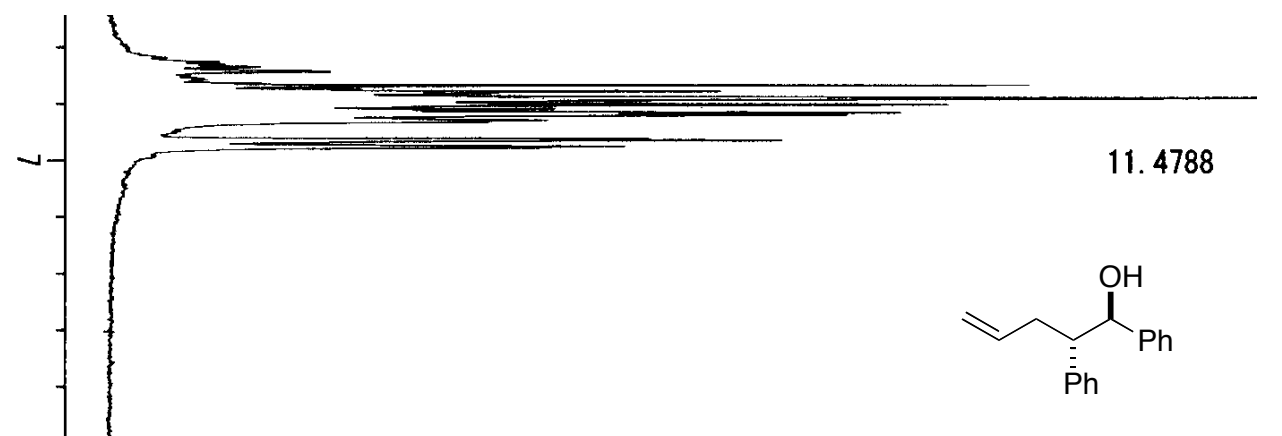

anti-1,2-Diphenyl-4-pentenol (4.3)

3.0768

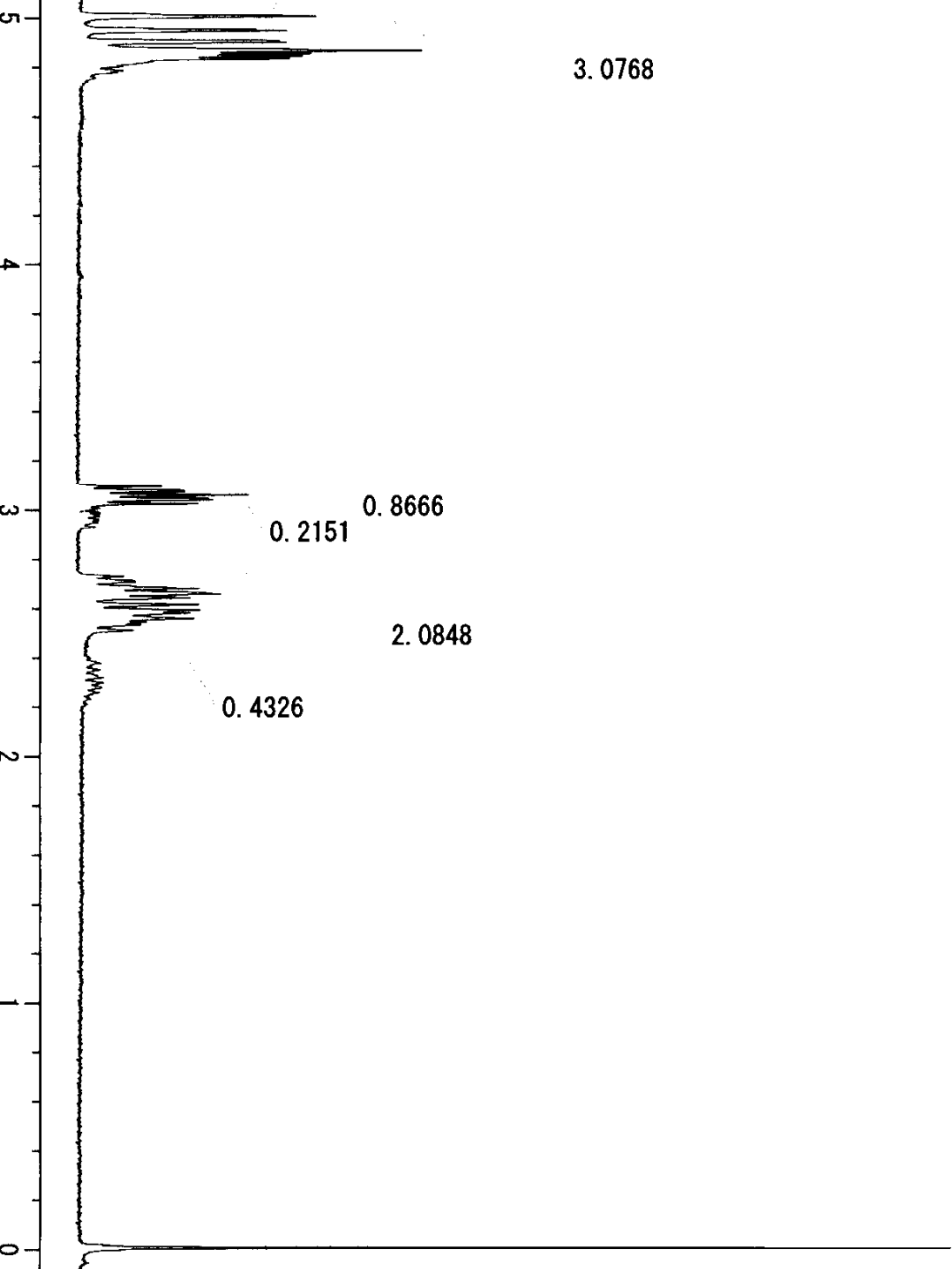




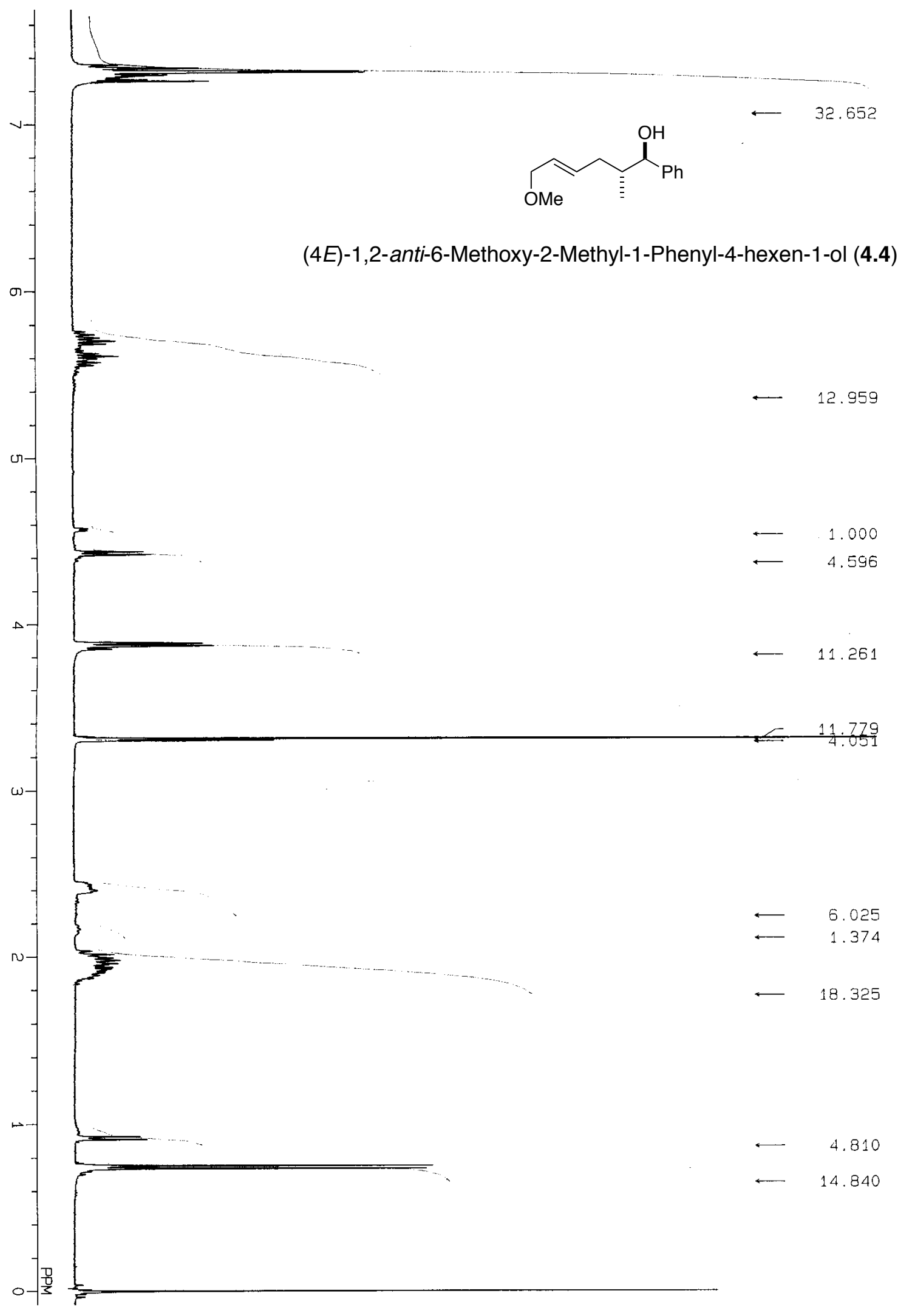




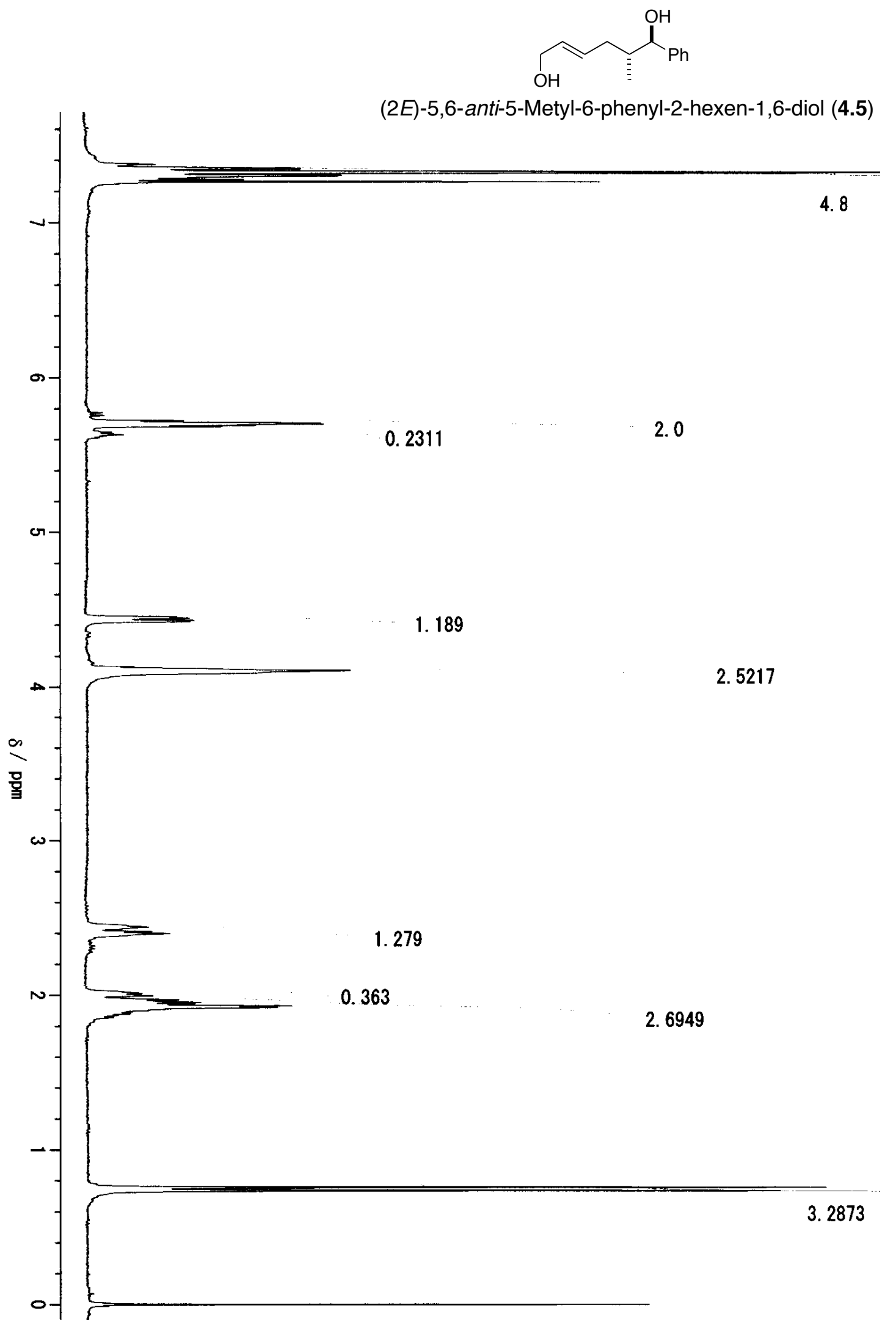




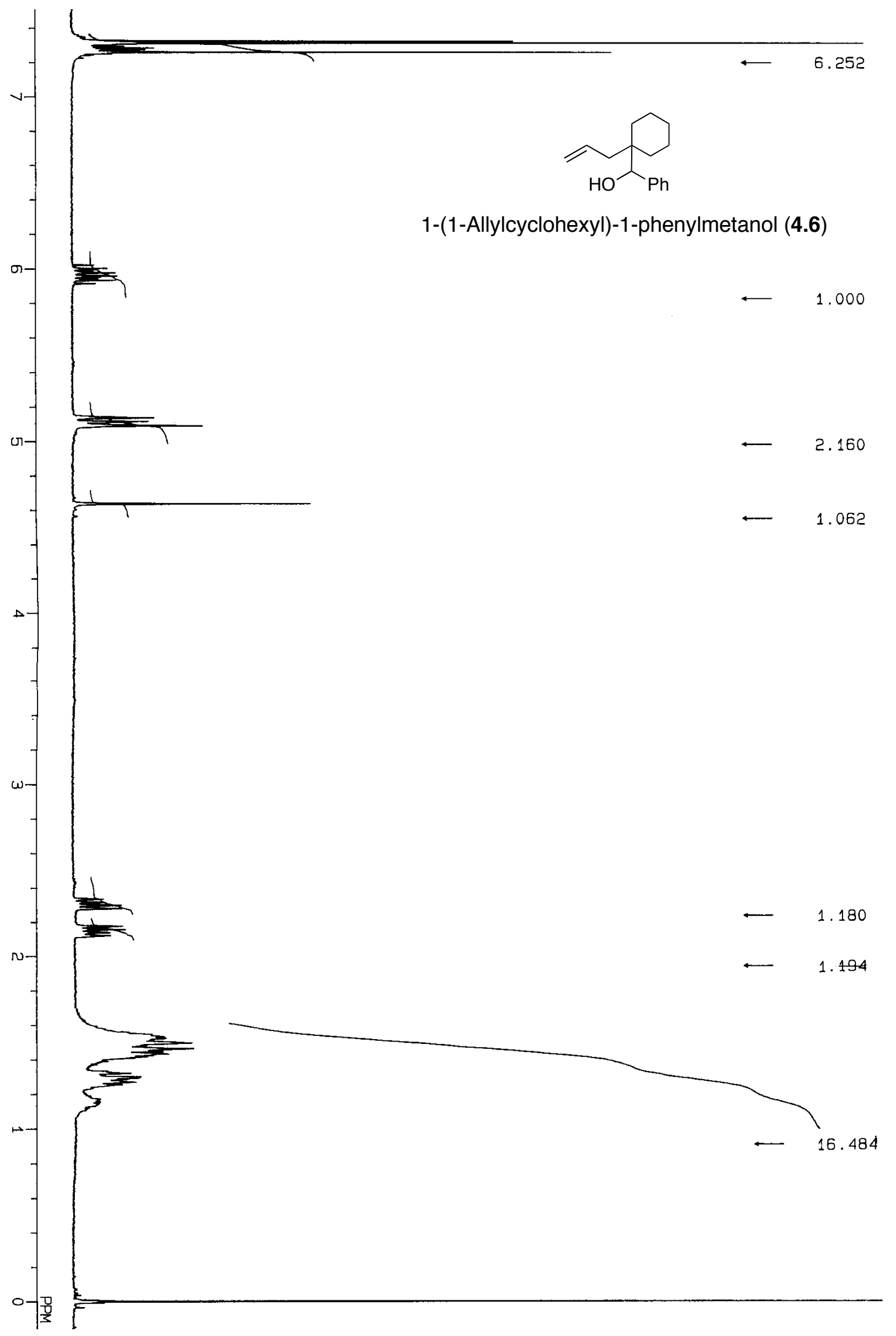




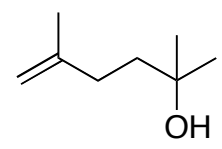

2,5-Dimethyl-5-hexen-2-ol (4.7)

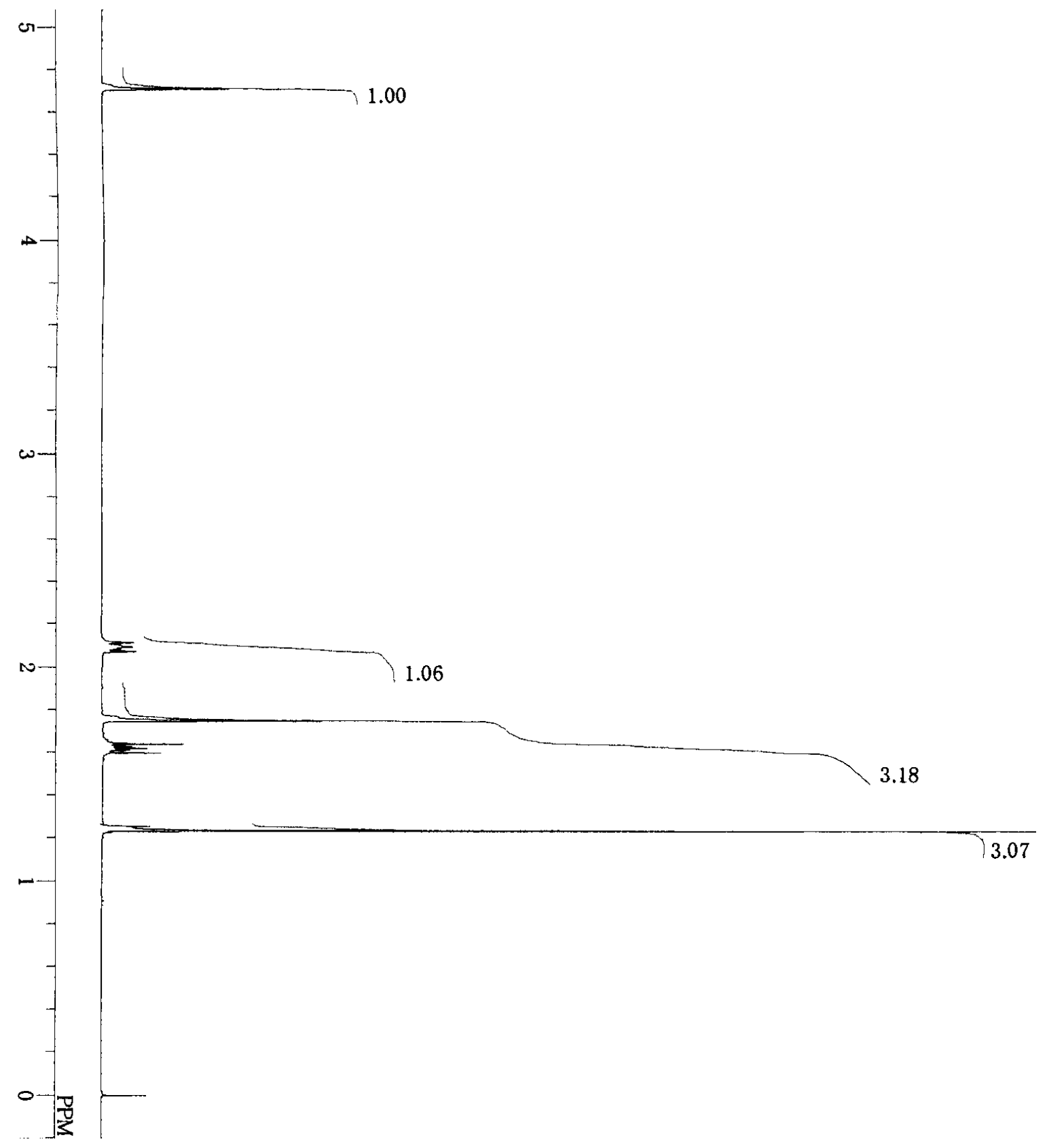




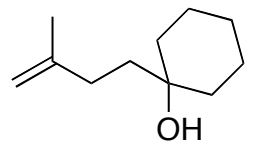

1-(3-Methyl-3-butenyl)cyclohexan-1-ol (4.8)

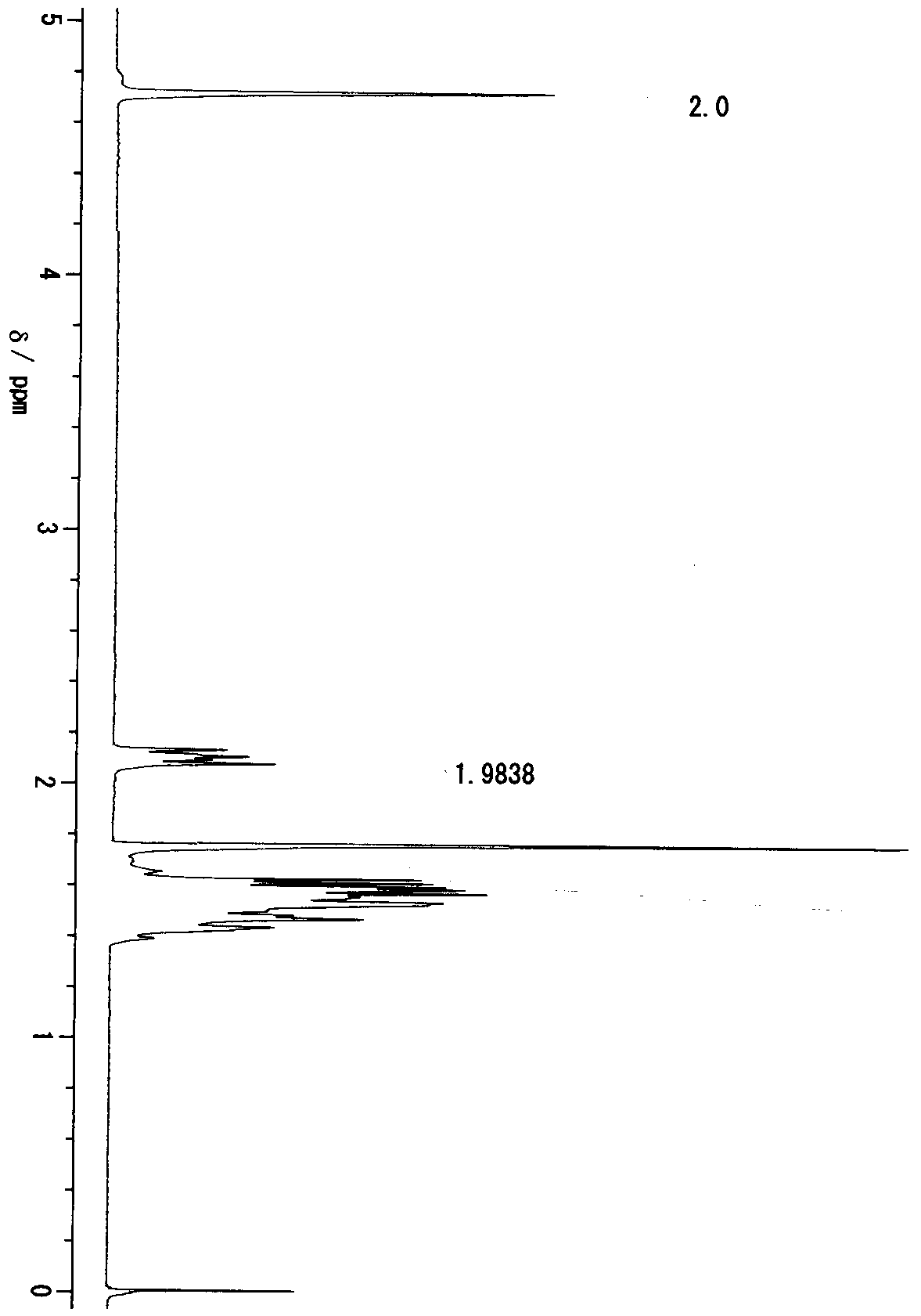

3. 0278

13. 5017 


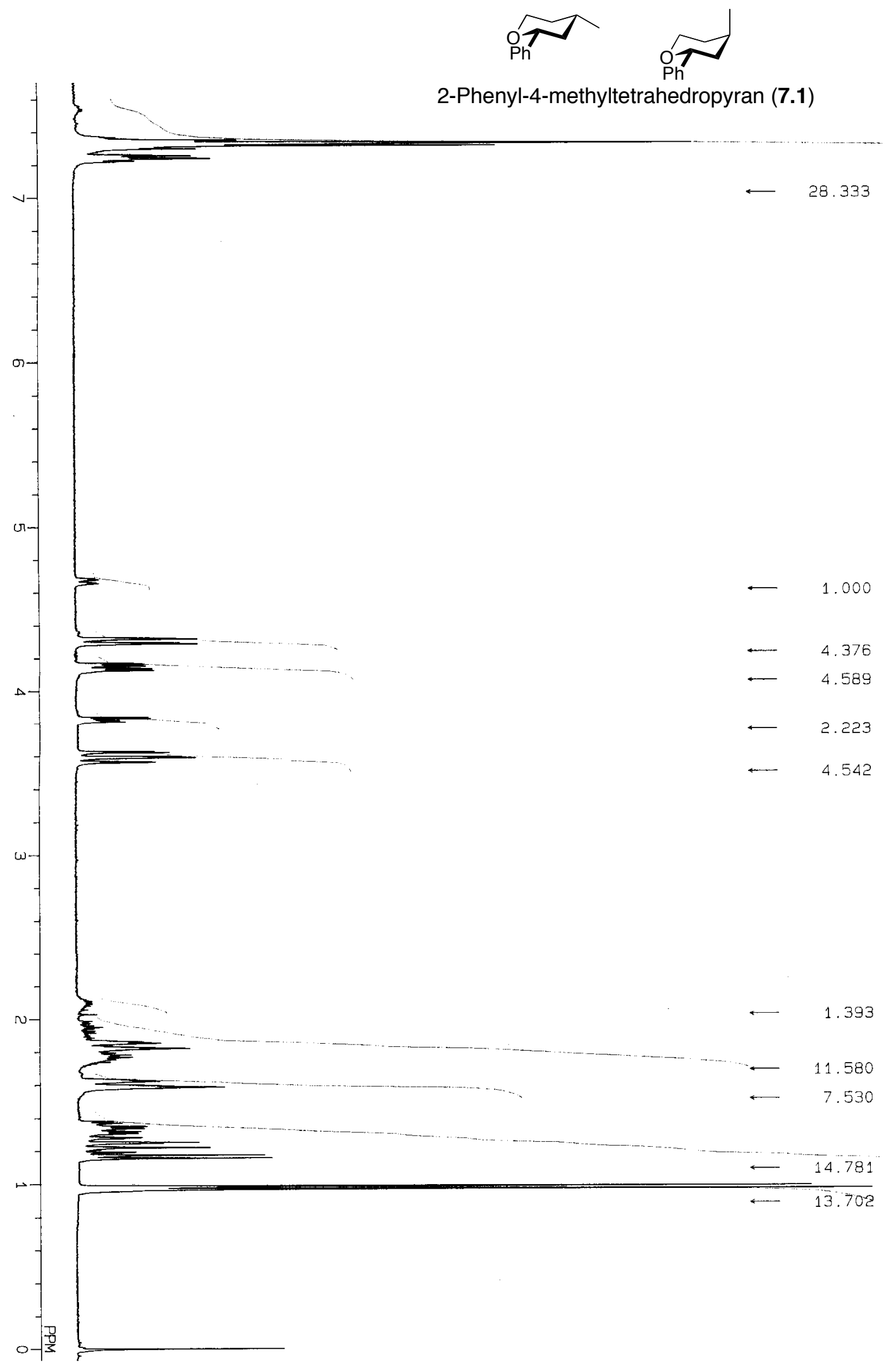




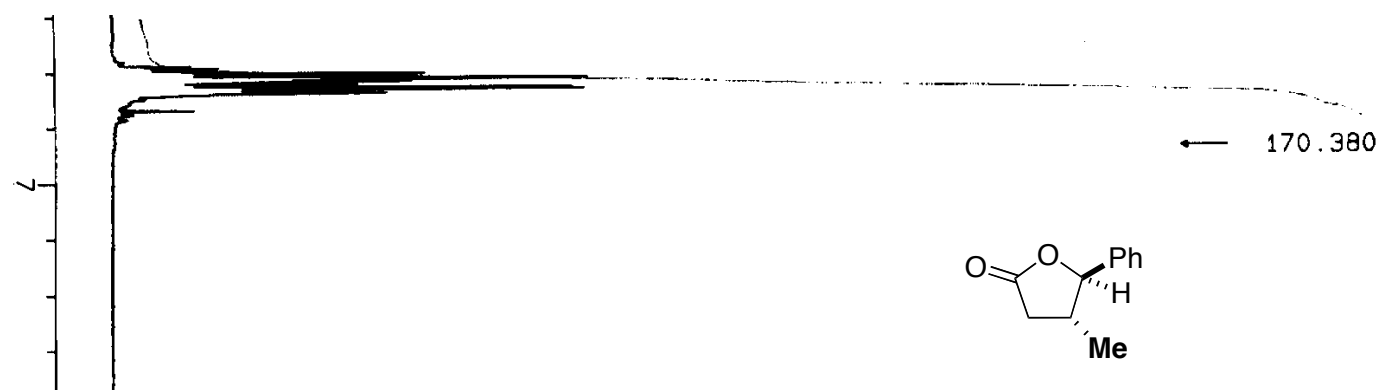

trans- $\beta$-Methyl- $\gamma$-phenyl- $\gamma$-butyrolactone (7.2)

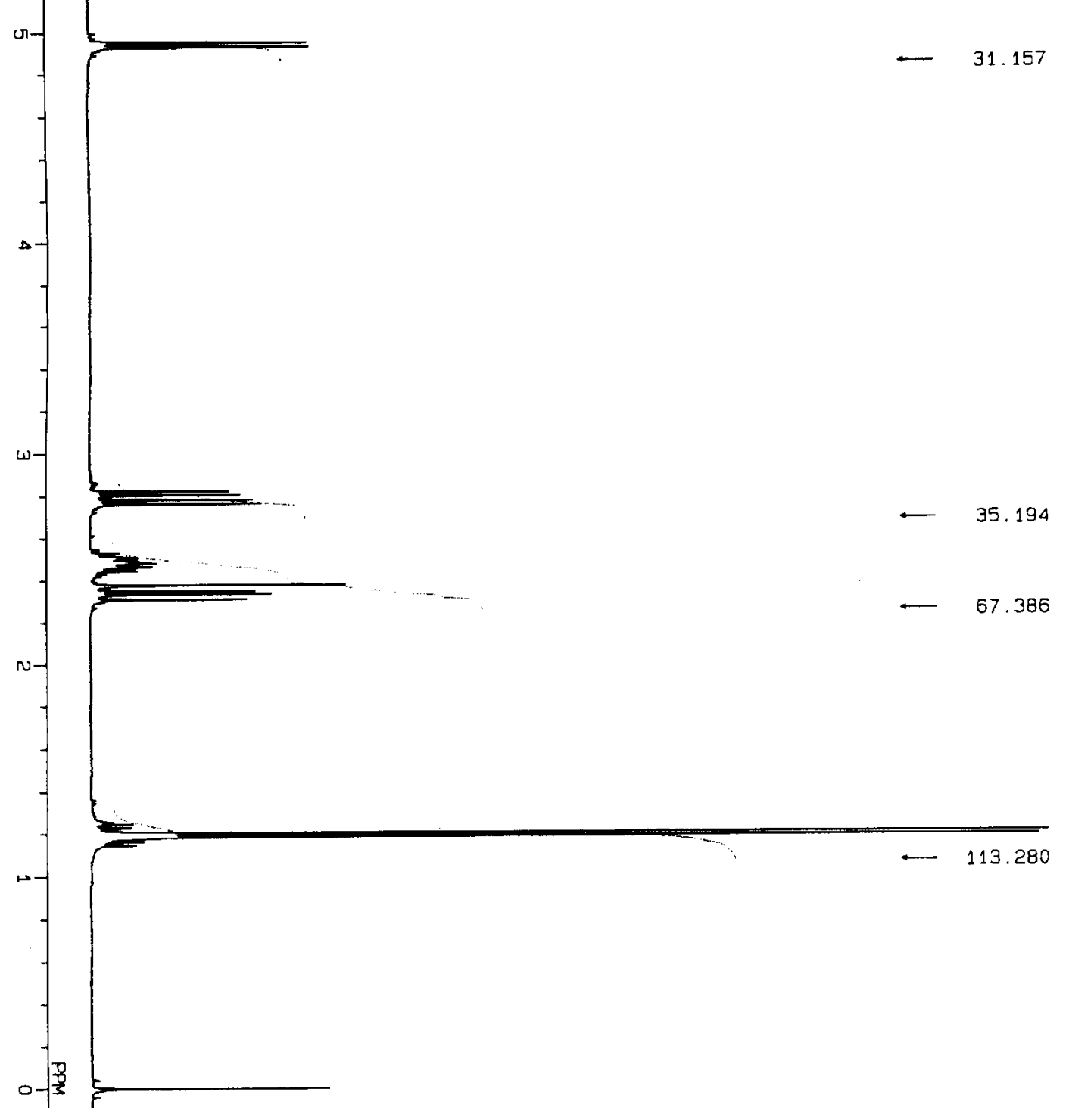




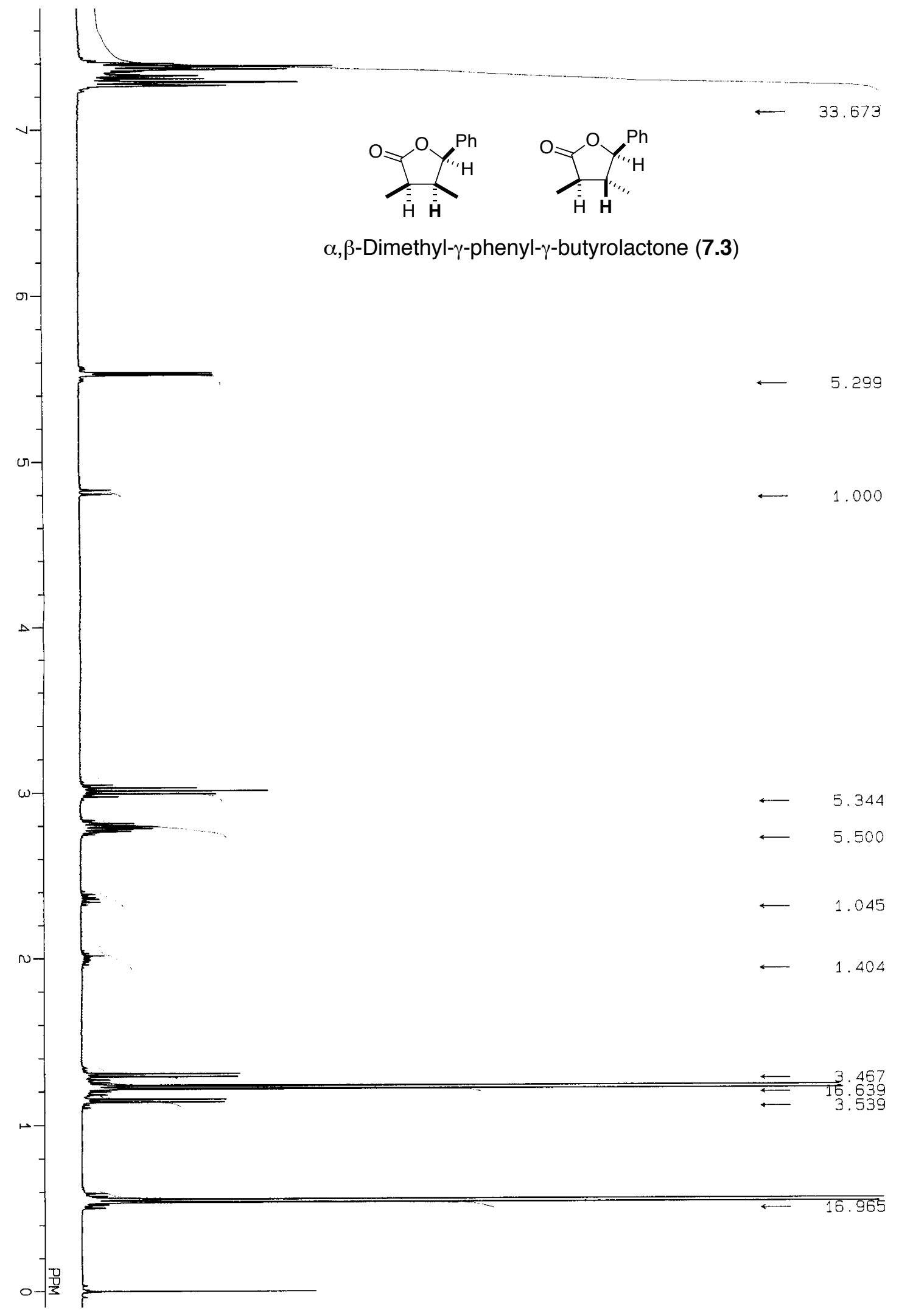




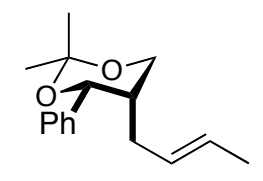

cis-5-((2E)-Butenyl)-2,2-dimethyl-4-phenyl-1,3-dioxane (7.4)

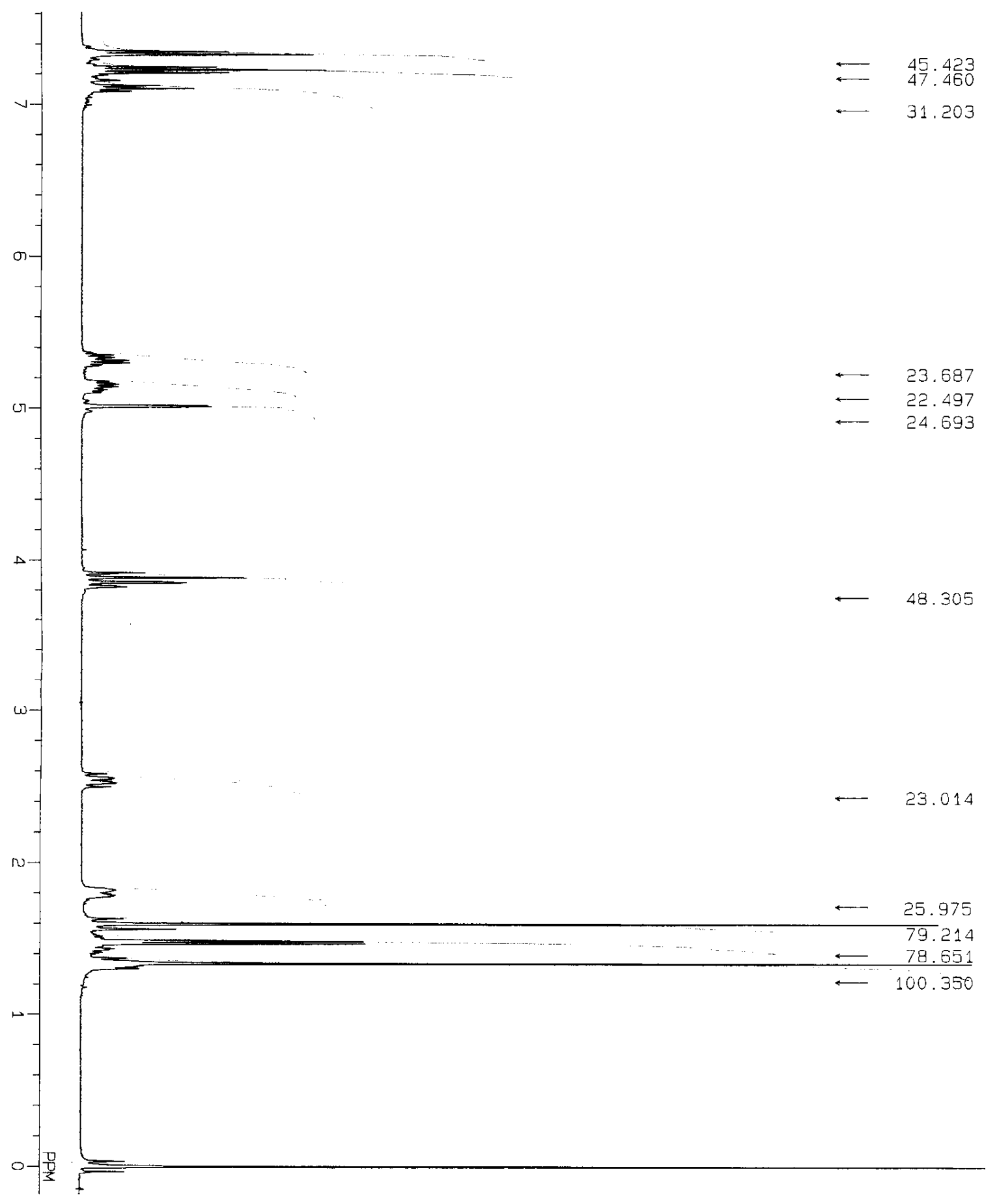




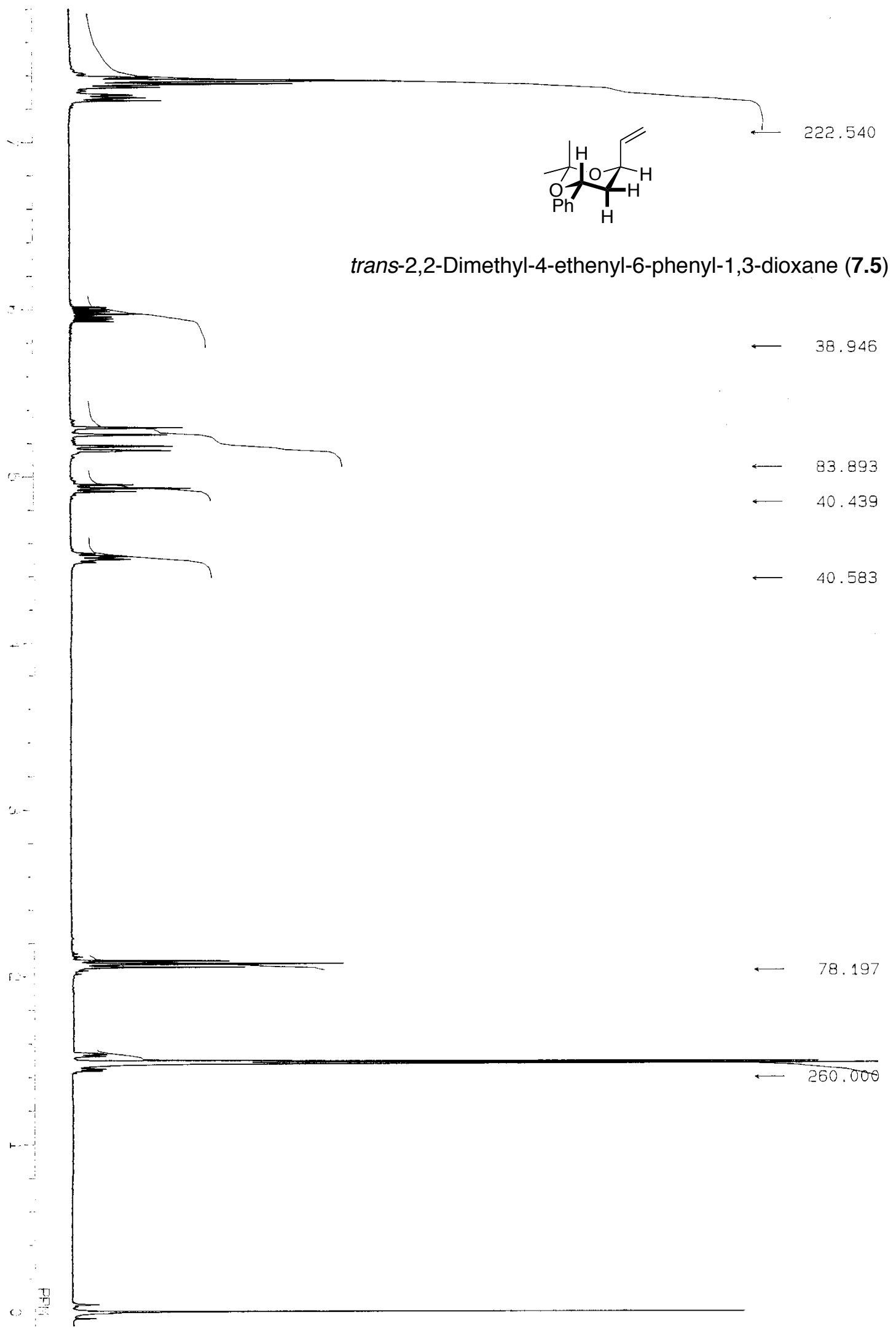




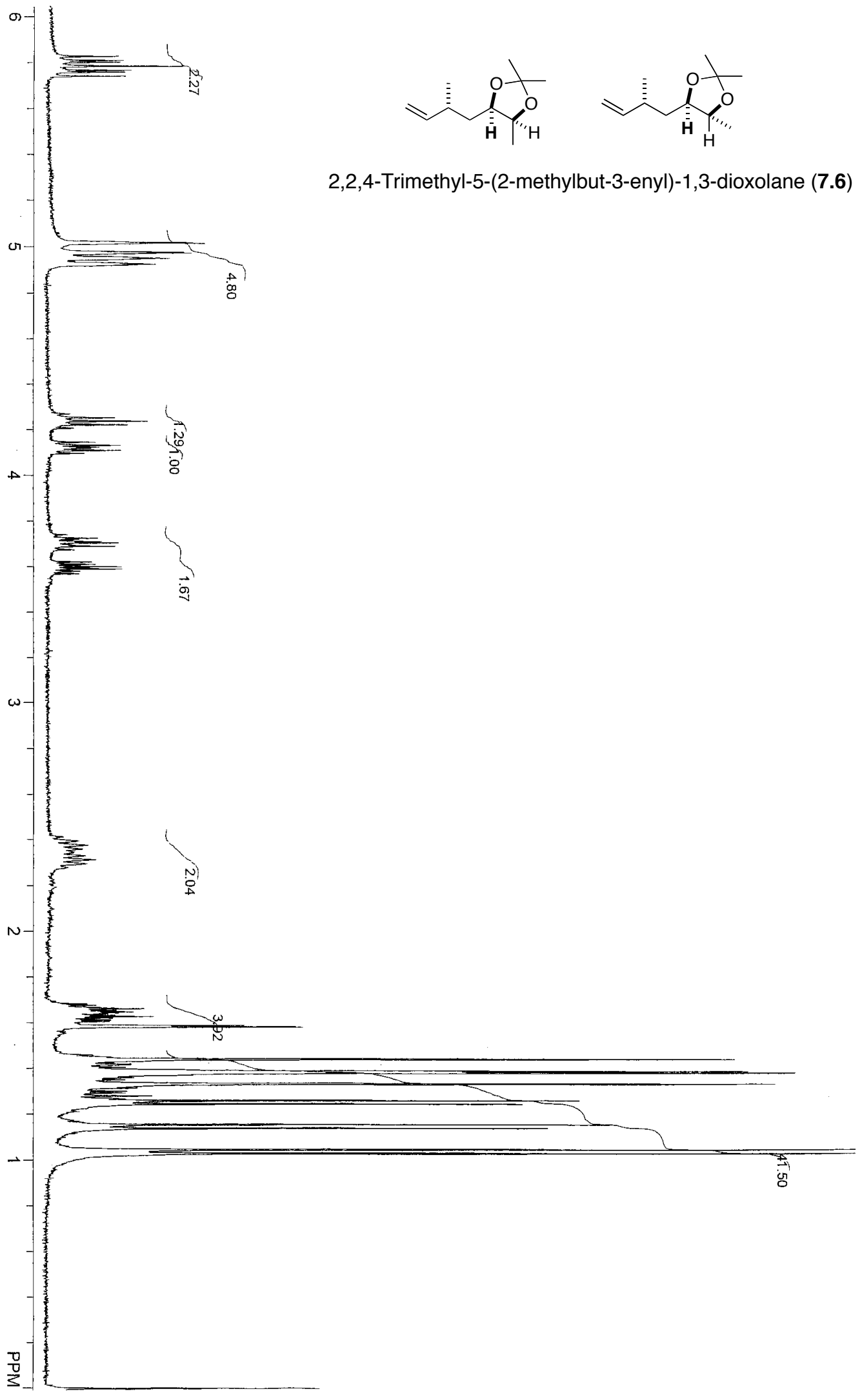




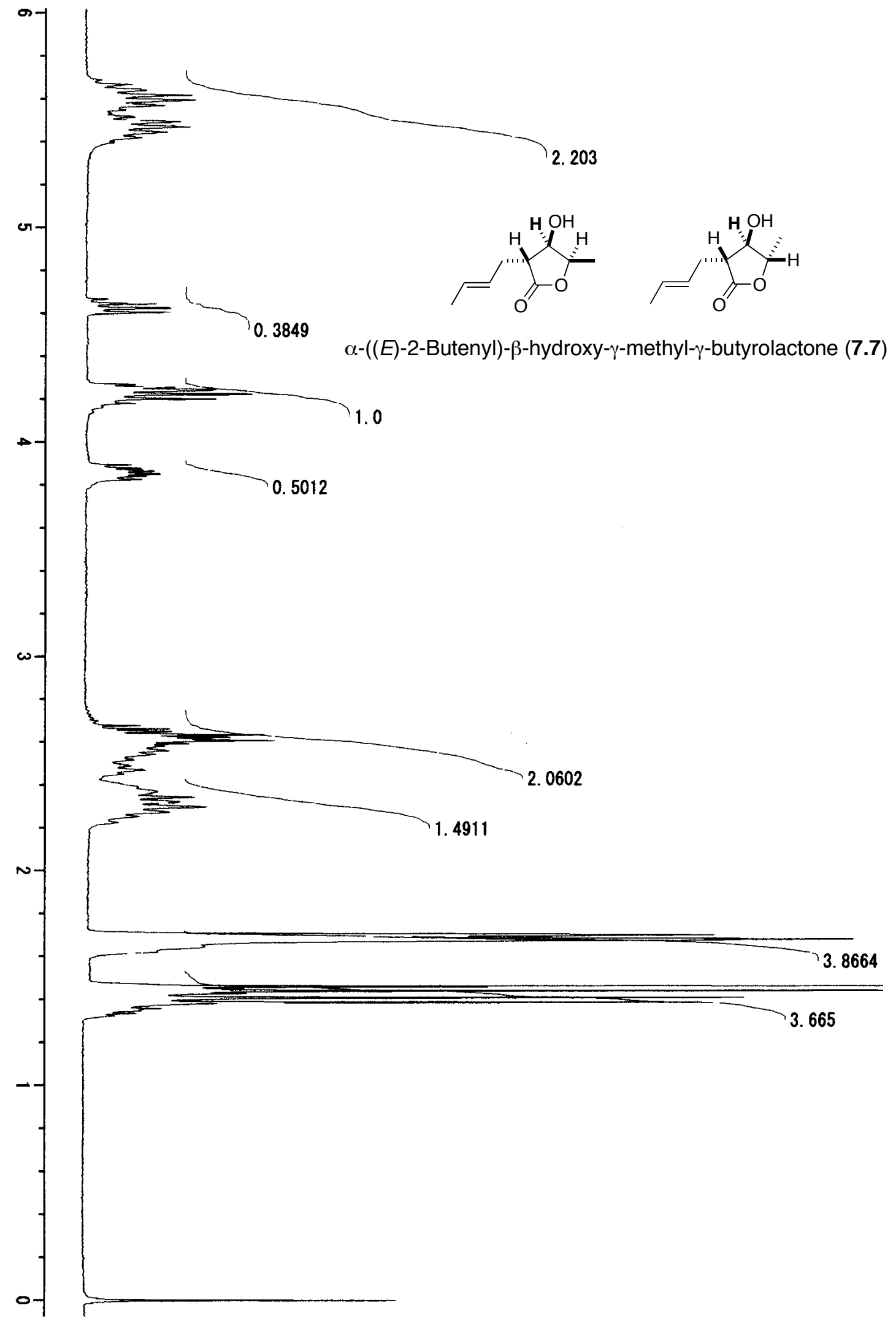




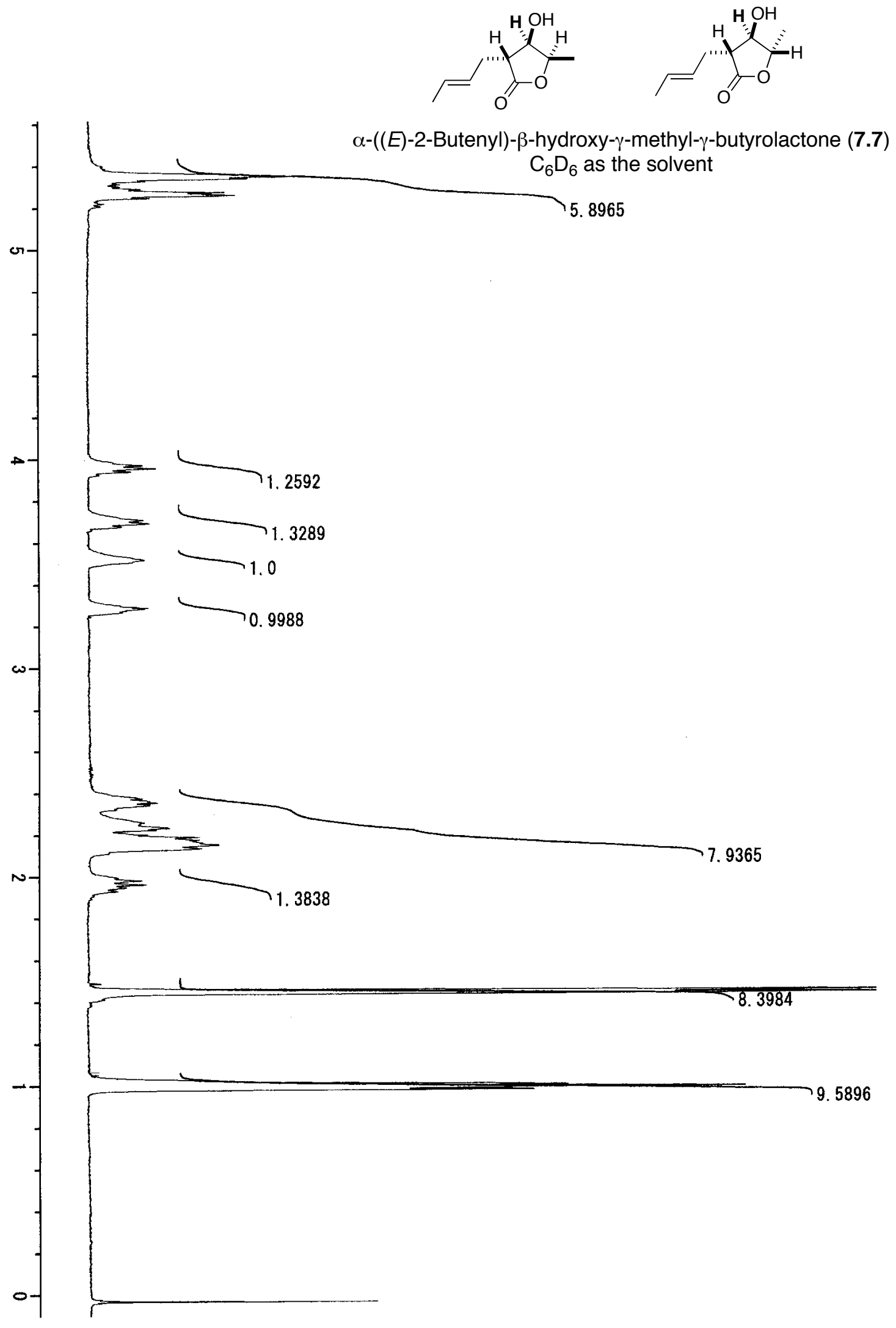

